\title{
OBSERVATION OF CIRCULATION IN ROTATING \\ SUPERFLUID HELIUM
}

Stephen Francis Kral

\section{Solid State and Low Temperature Physics Group}

\section{SCHOOL Of PHYSICS AND ASTRONOMY}

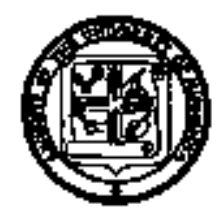

March 1973

UNIVERSITY OF MINNESOTA

MINNEAPOLIS, MINNESOTA

Work Supported in Part by the U.S. Atomic Energy Commission

Contract AT (11-1) - 1569

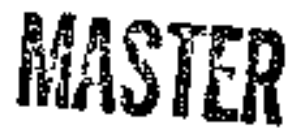




\section{DISCLAIMER}

This report was prepared as an account of work sponsored by an agency of the United States Government. Neither the United States Government nor any agency Thereot, nor any of their employees, makes any warranty, express or implied, or assumes any legal liability or responsibility for the accuracy, completeness, or usefulness of any information, apparatus, product, or process disclosed, or represents that its use would not infringe privately owned rights. Reference herein to any specific commercial product, process, or service by trade name, trademark, manufacturer, or otherwise does not necessarily constitute or imply its endorsement, recommendation, or favoring by the United States Government or any agency thereof. The views and opinions of authors expressed herein do not necessarlly state or reflect those of the United States Government or any agency thereof. 


\section{DISCLAIMER}

Portions of this document may be illegible in electronic image products. Images are produced from the best available original document. 


\author{
OBSERVATIOH OF CIRCULATION IN ROTATING \\ SUPERFIUID HELT UM
}

\title{
A Thesis
}

SUBMITTED TO THE FACULTY OF THE GRADUATE SCHOOL OF THE UAIVERSITY OF MINNESOTA

By

STEFHEN FRANCIS RRAL

IH PARTIAL FULFILLMEHT OF THE REQUIREHENTS

FOR THE DEGREE OF

DOCTOR OF PHILOSOPHY

MARCH; 1973

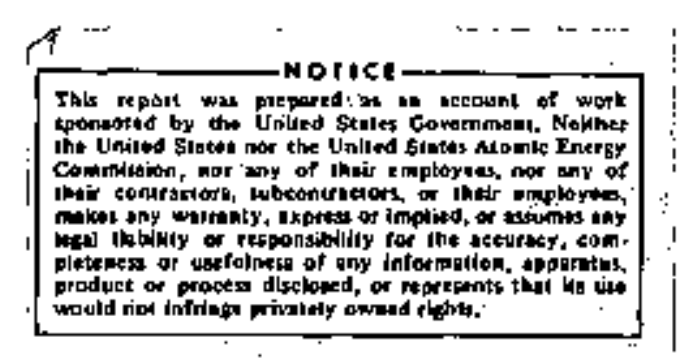

\section{MMSTER}




\section{ABSTRACT}

Measurements of the circulation of the superfluid component of the II around a fine wire stretched along the axis of a cylindrical container have been made while the contalnet and wire were In rotation. Theoe measurements form an extersion Df earlier measurenente made in our laboratory by whitoore and ziamerwann (l) of the clrculation around fine wire in a similar contatner, but with the container and wire at reat.

The method of W. F. Vinen (2) is used,in which the cfrculation is measured by means of the influence it has on the transverse vibrations of the wre.

Measurements were made at temperatures near to $1.30 \mathrm{k}$ for rotation apeeds in the range from 0 to $\pm 4 \mathrm{rad} / \mathrm{gec}$, and for wire diameterg ranging frow 60 to $90 \mathrm{~m}$. Both the magnitude and direction of the circulation were obtained.

Two principal results are found. The firgt is that at a given rotation speed the circuiation exhiblts a high degree of metastabilicy, with the metasable values found belag at both integer and 
non-1nteger multiples of the circulation quantum h/m. In addition, at a given rotation speed the metastable values cover a range of circulation values, and the center value of this range increases as the rotation speed increases. Generaliy, circulation values in the lower part of the range are observed as the rotation speed ls increased and circulation values in the higher part of the range are observed as the rotation speed 18 decreased. With the exception of a small region around the zero of the rotation speed, the circulation has the same sense as the rotatiun. The second principal result is that as the rotation speed is changed continuously the circulation exhibitg a serieg of step-11ke changes between netastable leve1s. These netastable levels appeat at both integer and non-integer multiples of the circulation quantum.

The range of circulation values measured at rest agrees with that seen by Whitmore and Zimmermann with wires of simflis diameter, although the clear-cut evtdence for quantization of c1rculation found in their work is lacking here. The high 
degree of metabtability found in the clrculation values measured appears to be related to the exterded period of time over which measurements were made on a particular sample.

The circulation valuea measured have been compred to a simple contiaum model for the free energy proposed by stauffer and Fetter. (3) it is found that the circulation valueg predicted by the theory lie within the range of metastable values heasured. Th1s apparent agreement between the theory which ts based upon the extstence of quantized vorticeg in the superfluid and the experimental results gives indirect support to the 1dea of quantized circulation in superflutd hellum. However, direct support for this ldea is not found in our work.

The fluid dyamics of the superfluid which gives rise to the high degree of unselective metastability observed is not vell understood. This metasability is possibly due to quantum vortices pinned to or near the wire, but no dicect evidence of this 19 obtalned from ow meagurements. 


\section{ABSTRACT REFERENCES}

1. S. C. Whitmore and W. Zimmermann, Jr., Phys, Rev, 166; iBj (1968).

2. W, F, Vinen, Proc, Roy, Soc, (London) A260, $218(1961)$ :

3. D. Stauffer arid A. I, Fetter, Phys, Rev. 168, 156 (1968). 
CONTENTS

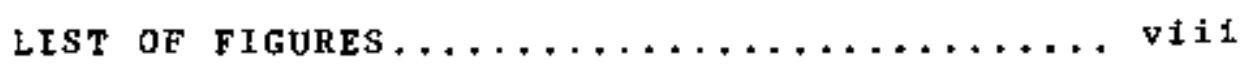

LIST 0 T $\operatorname{TABLES} \ldots \ldots \ldots \ldots \ldots \ldots \ldots \ldots \ldots \ldots \ldots$

I . INTRODUCTION ................. 1

1. Vortices and Quantized circulation in Superflutd Helium............ 1

2. Technique................ 4

II. THE MOTION OF THE WIRE ........... 9

1. Introduction ..............

2. The Hydrodynamic Forces........ 9

3. The Normal Fluld............ 10

4. The superflu1d............ 13

5. The Motion of the wire........ 1s

6. Motion of the Wire in a Non-Rotacing coordinate Frame with Zero circulation................ 17

7. The Mation of the Wire for Nonzero Circulation and in Rotation.. 21

8. The Normal-Made Equations...... 34

9. Observation of the Motion....... 38

10. Model Calculstion........... 39

11. Application to an Asymetric Wire. 45

12. The Direction of Circulation.... 47

13. Experimental Considerations..... 49 


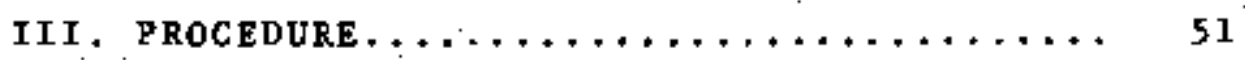

$\$$

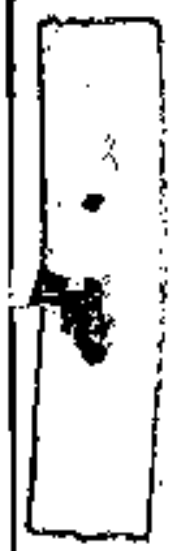

1. Description.............. sl

2. The Wire Drive signal........ 59

3. Determination of the Intrinsic Frequency Splitting........... 62

4. Data Reduction and Analysis...... 64

IV. Restrts ................... 69

1. Results............... 69

2. Attenpte to change Citculation

Levels.................... 85

3. Runs With Congtant Acceleration... 88

4. Errors ................. 89

5. Errors in Measured Quantities..... 90

v. THEORY AHD DISGUSSION . . . . . . . . 9

1. Equilibrium Theory............ 99

2. Discussion............... 106

\section{AF PENDI CES}

I. EXPERIMENTAL APPARATUS .......... III

1. Introduction............... 111

2. Overall Apparatus........... 111

3. Cryogenic Features of the

Apparacus.................. 116

4. The He $\mathrm{H}^{4}$ pot............... 117

5. The $\mathrm{He}^{3}$ Pat............... 118

6. Thernometry............... 123 


$$
\text { vi }
$$

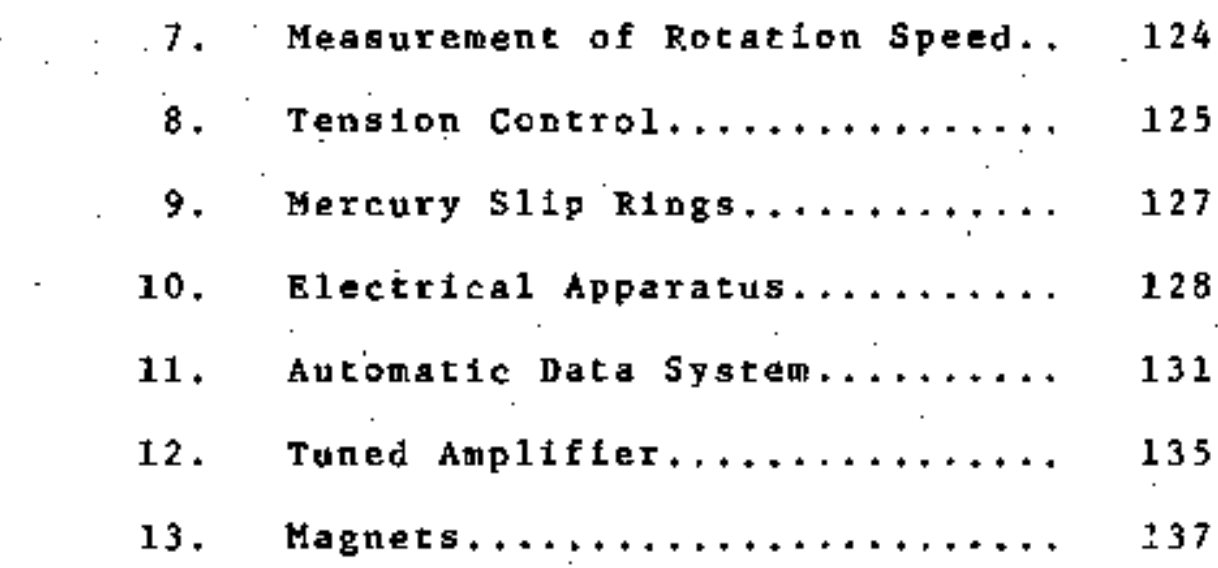

II WIRE PREPARATION .............. 139

1. Making The Wire.......... 139

2. Mounting The Wire.......... 140

3. Wire set-Up Bur........... 142

REFERENCES . . . . . . . . . . . . . . . . 149

ACKNOWLEDGMENT . . . . . . . . . . . . 153 


\section{LIST OF FIGURES}

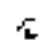

1. Overall Schematic of Apparatus....... 5

2. The Experimental Ce11...........

3. Schematic of Lower Part of Apparatus.. S2

4. Circulation vs. Angular velocity of Rotation for Runs with Wire A...... 75

5. Circulation vs. Angular Velocity of Ratation for Runs With Wire B.......7 76

6. Time to First Hade vs. Time Inco Run For Run $\mathrm{C}-3 \ldots \ldots \ldots \ldots \ldots \ldots \ldots \ldots 7$

7. Circulation vs. Time Into Run For Run $c-3 \ldots \ldots \ldots \ldots \ldots \ldots \ldots$

8. Circulation vs. Angular Velocity of Rotation For Run C-3.......... 79

9. Circulation ve. Time Into Run For Run $c-5 \ldots \ldots \ldots \ldots \ldots \ldots$

10. Circulation vs. Angular velocity Rotation For Run $C-5 \ldots \ldots \ldots \ldots \ldots \ldots$ 8

11. Block Dlagram of Electrical systems... 130

12. Detal1 of Upper Post.............. 140

13. Node Time vo. Tuist For Wire B...... 144

14. Comparison of Node Time vs. Wire Frequency for Wire $B$ During set-UP

Run and Regular Run.............. 146 


\section{LEST OF TABLES}

w

Ia. Experimental parameters for Runs with Wire A........................ 70

Ib. Experimental Parameters for Runs with

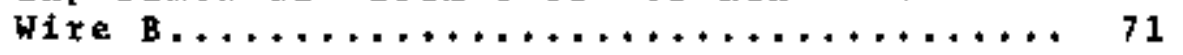

Ic. Experimental Parameters for Runs with

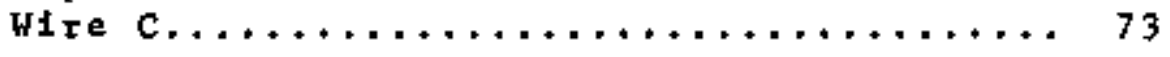


CHAPTER I

I N T RODUCT I ON

1. Vortices and Quantized circulation in Superfluid Helium

Landau, (I) in his development of the two-fluid hydrodynamical nodel of He II, proposed that the flow of the superfluid is subject to the constraint that the superfluid velocity field is irotational, i.e., that

$$
\cos 1 \vec{v}_{s}=0 . \quad 1-1
$$

Experimentally it is found that the macroscopic rotational propertieg of He II sugbest a fluid in rotational flow. $(2,3)$ In order to maintain the condition expressed in Eq. 1-1, and at the same tme explain the experimental results, several autbors, principaliy Feynan, (4) and onsager, (5) have suggested that rotating hellum is microscopically irrotational almost everywhere but is threaded by an array of vortex 1ines. Further, these vortex lines are thought to have quantized circulstion, so that the line integral of the sugerfluid velocity field around a closed path containing a vortex is an integral multiple of the ratio $\mathrm{b} / \mathrm{m}$,

$$
\oint v_{s} \cdot d l=n(b / m), \quad n=0,1,2 \ldots \quad 1-2
$$


where h $1 \mathrm{~s}$ Planck's constant and to ts the mass of the $\mathrm{He}^{4}$ atom.

A number of experiments have been performed which verify the truth of this hypothesia. Perhaps the most direct verification of the existence of vortices in He II comes from the work of Rayfleid and Reif. (6) In this work 1 t was shown that the excitation which determines the motion of an entrapped fon has dynamical properties similar to those of the vortex rings of classical hydrodynamics. In addition, the circulation of the vortex ring was found to be one quantum unfl to a high degree of accuracy.

Many other experiments give indirect support to the notion of vortices with quantized circulation in He II, $(7,8,9)$

A direct method for measuring the ctrculation of the superfiuid in He II is that of vinen. (10)

He showed that if circulation is present around a vibrating wire, Magnus or lift force acts on the wre. This force couples the normal modes of vibration of the wire and gives rise to frequency splittings between otherwise degenerate palts of mades. By measuring the splitting between the lawest-frequency palc of normal modes the circulation 
can be decernined. In his experiment Vinen showed that the circulation was quantized and tended to take on a value of one quantum untt. Whitmore and zimmermann extended the work of Vinen. (11) Their work demonstrated that superfluid motion persiated around their wire for long periods of time even though the vessel containing the fluid was at rest. In addition, they found that the circulation of the flows they observed tended to be at integral multiples of the ratio $\mathrm{h} / \mathrm{m}$, with metastabie circhiation values as large as $3 \mathrm{~h} / \mathrm{m}$ being seen. They aloo found that larger circulation values were seen with wires of larger dianeter than were seen with wies of snali diameter.

Some observations made in the course of the work of Whitmore and zimeruinn suggested that frutiful studies of circulation could be made with the vessel containing the He II and the wire in rotation. For example, at rest the largest metastable circulation observed ras $3 \mathrm{~h} / \mathrm{m}$. However, much larger ctrculation values were seen lmediately after the system was brought to rest after rotation, suggesting that a wider range of circulation values could be seen in rotation. Also, the theory of rotating he II as applied to a cylindrical 
annulus predicts the dependence of the circulation on the angulat velocity of rotation. Measurements of this dependence would be a useful test of the theory of the rotating flutd. With these consideratlons in mind the work presented here was begun.

\section{Technigue}

The circulation measurements made in this work were made using the technique originally proposed by vinen. (12) To allow for measurements in rota-. ton, the apparatus ahown in Fig. 1 was constructed. This apparatus was basically a He ${ }^{3}$ cryostat which was mounted on a turntable which cotld be rotaced by means of a motor drive.

The experimental cell is shown in Fig. 2 . The wire used to measure the circulation was mounted so that it was coincident with the axis of rotation of the system. These wires were goldplated quartz fibers, approximately 0.1 mm in diameter and $50 \mathrm{~mm}$ in length.

A magnet was wounted on the epparatus and rotated with che apparatus. The field of this magnet was perpendicular to the axis of the wire. A short current pulse through the wire was used to excite the wire into tranaverge vibration. The wire was then allowed to oscillate freely. 


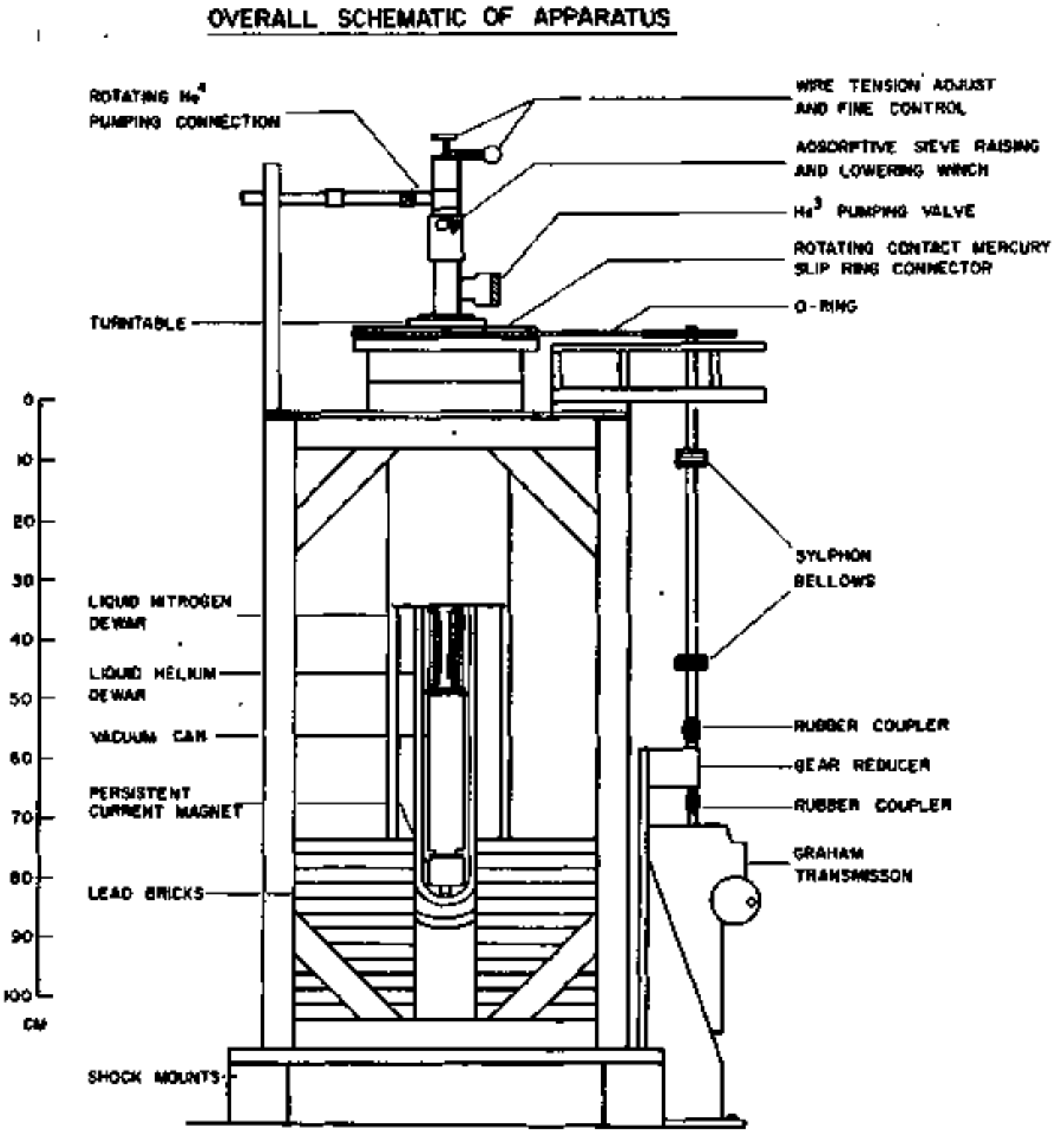

FT GURI? I 
The vibrations of the tre were observed by reans of the enf generated while it vibrated in the presence of the transperse magnetic field. The signal generated by the vibrating wire when displayed on an oscilloscope acreen was geen to be a decaying beat pattern. The beat frequency $\Delta_{t}$ was obtained from this sigual.

As is shown in Chapter II, the beats observed arise because of small frequency differences between the frequencies of the two lowest transverse modes of vibration of the wire. The differences, as. viewed in the rotating systen, come from three different sources. There is an intrinsic frequency difference which arises from asymetries in the wire and its mounting, which we call bw. The Magnus force which acts when circulation is present arotid the wire introduces a frequency splitting between the modes which we call $\Delta \omega_{K}$. This splitting Is shown in Chapter II to be related to an average of the circulation over the length of the wire, a quantity which we call the apparent circulation $\bar{k}$. The frequency splitting due to clrculation is given by $\Delta \omega_{k}=p_{g} \bar{k} / \nu$, where $\rho_{g}$ is the superfluid density and ts the effective mass per unit length of the wire. In addition, when the system is in rotation 


\section{EXPERIMENTAL CELL}

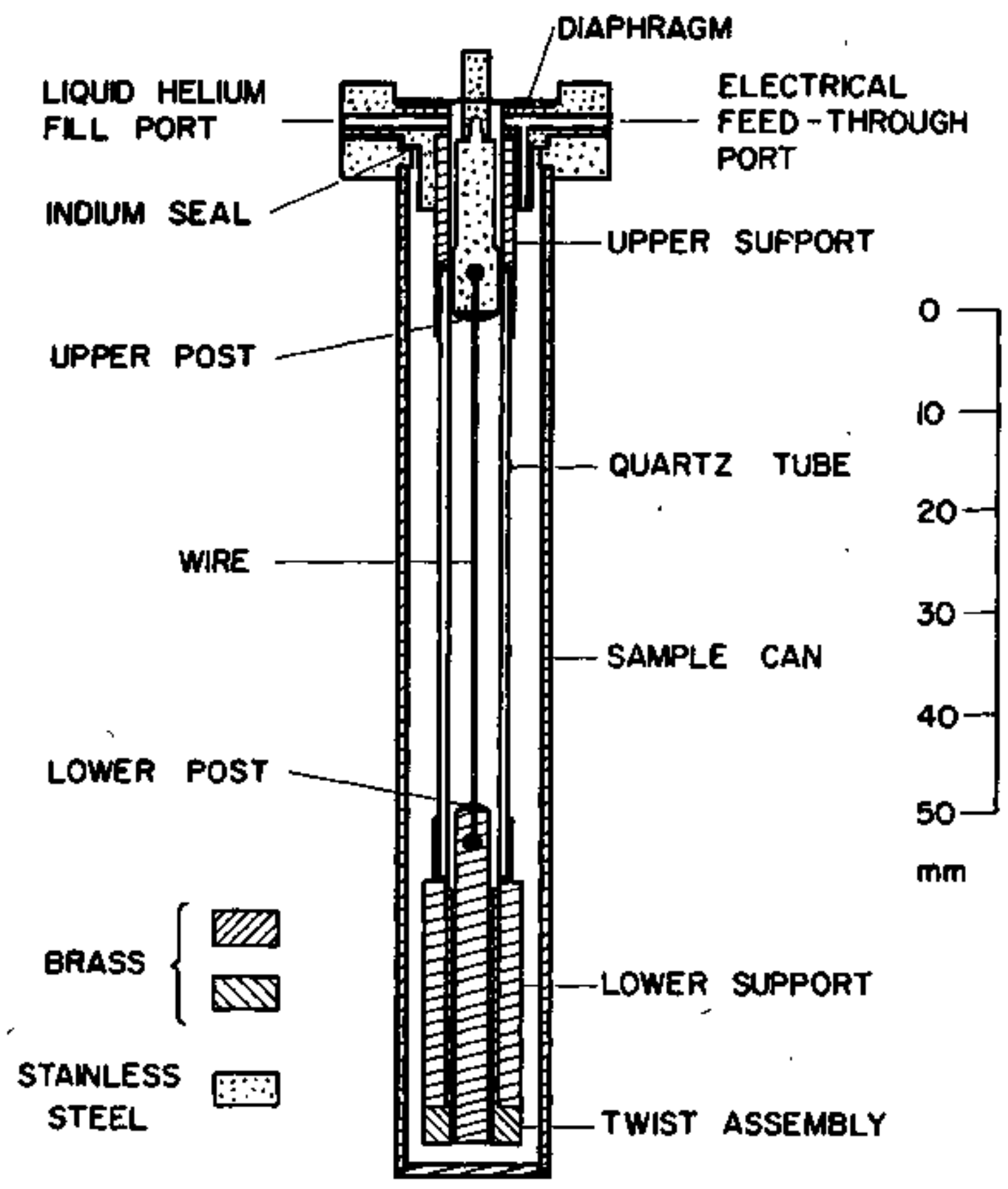

FIGURF 2 
the Coriolis force introduces a splitting given by $\Delta \omega_{r}=2 \omega_{r}$, where $\omega_{r}$ is the angular velocity of rotation. Our analysis of the dynamics of the wire's motion shows that the total frequency splitting is related to these splittings by the expression

$$
\Delta \omega_{t}=\sqrt{\left(\Delta \omega_{0}\right)^{2}+\left(\Delta \omega_{k}-\Delta \omega_{r}\right)^{2}} \cdot \quad 1-3
$$

Thus, measurements of the total frequency splitting can be used to determine the apparent circulation around the wire.

In Chapter II a complete discussion of the dynamics of the wire's motion is presented. Chapter III is a discussion of the procedures followed in making circulation measurements and analysing the data. Chapter IV presents our results, with error analysis. Chapter V contains a discussion of our results and some suggestions for future study. Appendices describing the mechanical and electrical apparatus and the methods used to set up the wire for measurement conclude the report. 


\section{CHAPTER II}

THE MOTION OF THE WIRE

\section{Introduction}

In this chapter the hydrodynamic forces which act on an asymmetric wire on the axis of a cylinder filled with He II are discussed. The form of the forces in the case where the wire is vibrating but the cylinder and wire are not rotating is obtained. Then, assuming that the superfluid is circulationfree, the solutions to the equations of motion of the wire are found. Using these solutions as a basis, solutions to the equations of motion of the wire are obtained when it and its containing cylinder are in rotation, and when circulation is present. Finally, we discuss the experimentally observed motion. 2. The Hydrodynamic Forces

To determine the forces a fluid exerts on a small wire vibrating on the axis of a cylinder containing He II, it is necessary to obtain solutions to the Navier-Stokes equation for the accompanying motion of the fluid. This has been done using the two-fluid model of He II. (13) This model enables one in the present case to reduce the problem to 
two separate problems, one concerned solely with the effect of the normal fluid on the wire, and one concerned with the effect of the superfluid on the wire. The separation depends upon the fact that here the two fluids may be treated as if they were each incompressible. This treatment is justifled by the fact that the fluid velocities treated in the experiment were much less than the velocities of first and second sound and that the times required for first and second sound to propagate across the cylinder were much smaller than the period of wire's vibration.

\section{The Normal Fluid}

In the case of the normal fluid, the problem is treated with the following assumptions:

(1) In the non-rotating state, the motion of the normal fluid is simply due to the vibration of the wire itself, and at the surface of the wire the fluid velocity is equal to the wire velocity; in rotation the normal fluid rotates with the cylinder in rigid body rotation (except for the vibrational motion), and at the surface of the wire the fluid velocity is equal to the wire velocity.

(2) Each element of length of the wire may be treated separately, as an oscillating cylinder in two dimensions. 
(3) The rate of change of vorticity generated in the normal fluid at the surface of the wire due to the shearing effects of the normal fluid is primarily due to diffusion, rather than to convection.

We first consider the case where there is no rotation. Then with these assumptions the force on the wire per unit length due to the normal fluid is found to be $(14,15)$

$$
\vec{F}_{n} / L=-\mu_{n} K \frac{d \vec{u}}{d t}-\mu_{n} \omega K \cdot \vec{u}, \quad 2-1
$$

where

$$
K=1-\frac{4}{\beta} \operatorname{Im}\left(\frac{\sqrt{1} H_{1}^{(1)}(\sqrt{1} \beta)}{H_{0}^{(1)}(\sqrt{1} \beta)}\right) \quad 2-2
$$

and

$$
K^{\prime}=4 / \beta \operatorname{Re}\left(\frac{\sqrt{i}{ }_{1}^{(1)}(\sqrt{1} B)}{H_{0}^{(1)}(\sqrt{i} \beta)}\right) .
$$

In these expressions $\vec{u}$ is the velocity of the cylinder vibrating at frequency $\omega$, given by $\vec{u}=$ $\operatorname{Re} \vec{u}_{0} e^{i \omega t}, \mu_{n}$ is the mass of the normal flufd displaced by a unit length of the cylinder of radius a as given by $\mu_{n}=\pi a^{2} \rho_{n}$, and $\beta$ equals $a\left(\omega / v_{n}\right)^{1 / 2}$, where $v_{n}$ is the normal-fluid kinematic viscosity. $\mathrm{H}_{1}^{(1)}$ and $\mathrm{H}_{0}^{(1)}$ are Hankel functions. $(16,17)$ For $B \geqq 1, K$ and $K^{\prime}$ can be approximated to better than $5 \%$ by the series expansions 


$$
\begin{array}{ll}
K \cong 1+\frac{2 \sqrt{2}}{\beta}, & 2-4 \\
K^{\prime}=\frac{2 \sqrt{2}}{B}+1 / \beta^{2} . & 2-5
\end{array}
$$

Since in our work $B \geq 4$ these approximations are quite adequate.

The force per unit length exerted on the cylinder by the normal fluid is thus seen to be a sum of two terms, one proportional to the acceleration, but oppositely directed, and one proportional to the velocity. The former term is the reaction to the force which the cylinder must exert in order to accelerate the fluid through which it moves. Its effect is simply to add to the cylinder's inertia. The factor ${ }_{{ }_{n}} \mathrm{~K}$ is called the hydrodynamic mass per unit length of the cylinder. In the limit in which $v_{n}$ goes to zero we note that $K$ goes to one and that this factor becomes exactly equal to the mass of normal fluid displaced by the cylinder. For the viscous fluid the term is slightly larger, due to the fact that the cylinder accelerates fluid by viscous drag as well as pressure forces. The 1atter term is proportional to the velocity and directed opposite to it. This force is a viscous dissipation force, and vanishes in the limit in which v ${ }_{n}$ goes to zero. 
These results apply to a cylinder vibrating in an unbounded fluid. If the fluid is bounded by an outer cylinder, the form of the force expression remains the same. However, both $K$ and $K^{\prime}$ must be corrected. These constants take the form

$$
\begin{aligned}
& K_{b}=\left(1+\alpha^{2}+2 \sqrt{2} / \beta\right)\left(1-\alpha^{2}\right)^{-1}, \quad 2-6 \\
& K_{b}^{\prime}=\left(\frac{2 \sqrt{2}}{\beta}+\frac{1}{\beta^{2}}\right)\left(1+\alpha^{2}\right),
\end{aligned}
$$

where $\alpha$ is the ratio of the inner to the outer radius of the cylindrical annulus. (18) In this experiment $\alpha$ is of order $10^{-2}$, and this correction is negligibly small.

Finally, the force due to the normal fluid has been calculated for the case of a circular section. In this experiment the wires were of slightly elliptical section. In this case, the force retains the same form, with small corrections entering because of the wire's small ellipticity. $(19,20)$

\section{The Superfluid}

A determination of the forces acting on the wire due to the superfluid is made with the following assumptions:

(1) At the surface of the wire the normal component of the fluid velocity is equal to the normal 
component of the wire's velocity.

(2) The superfluid velocity field is the sum of the velocity field produced by the motion of the wire and that due to circulation of the superfluid. With these assumptions the force acting per unit length on a cylinder vibrating in an unbounded superfluid, with circulation present, is found to be

$$
\vec{F}_{s} / L=-\mu_{s} \frac{d \vec{u}}{d t}+\rho_{s} k \hat{n} \times \vec{u}, \quad 2-8
$$

where $\vec{u}$ is the velocity of the cylinder, $\mu_{g}$ is the mass of superfluid displaced by a unit length of the cylinder, and $k$ is the superfluid circulation around the cylinder. (21) The direction of the unit vector n is given by the right hand rule, with the fingers of the right hand indicating the direction of fluid motion. The circulation is defined to be the line integral of the superfluid velocity around any contour encircling the cylinder

$$
\kappa=\oint \vec{v}_{s} \cdot d \vec{l} \text {. }
$$

The first term of $2-8$ is an inertial term similar to that found in the force due to the normal fluid. The second term is the Magnus or lift force. It is shown by Lamb that the lift on the 
the cylinder is independent of the shape of the cylinder's section.

As for the normal fluid, in the case when the superfluid is bounded by an outer cylinder, the force takes a slightly different form, given by

$$
\vec{F}_{s} / L=\left(1-\alpha^{2}\right)\left[-\left(1+\alpha^{2}\right) \mu_{s} \frac{d \vec{u}}{d t}+p_{s} k \hat{n} x \vec{u}\right], 2-10
$$

where $\alpha$ is defined as before. As before, this correction is ignored.

5. The Motion of the Wire

The net force acting on a unit length of the wire due to the fluid is given by

$$
\begin{aligned}
\vec{F}_{s} / L+\vec{F}_{n} / L=-\left(\mu_{s}+K_{\mu_{n}}\right) \frac{d \vec{u}}{d t} \\
-\mu_{n} \omega K^{\prime} \vec{u}+\rho_{s} k \hat{n} x \vec{u} .
\end{aligned}
$$

We are interested in determining the transverse motion of a stiff wire under the influence of these forces.

For transverse vibration there are two equations of motion. Experimentally it has been found that in the absence of circulation the two normal modes of the wire of lowest frequency are plane-polarized, with mutually perpendicular planes of polarization! (23) It is natural to choose a coordinate system where the 
$x-z$ and $y-z$ planes correspond to the two perpendicular planes of plane polarization, with the z-axis along the wire axis. In this coordinate system, assumed non-rotating, the equations of motion of the stiff wire including the forces due to the fluid are

$$
\begin{aligned}
& \mu \frac{\partial^{2} x}{\partial t^{2}}=T \frac{\partial^{2} x}{\partial z^{2}}-S x \frac{\partial^{4} x}{\partial z^{4}}-2 \mu_{x} \lambda x \frac{\partial x}{\partial t} \\
& -K \rho_{s} \frac{\partial y}{\partial t}, \quad 2-12 a \\
& u_{y} \frac{\partial^{2} y}{\partial t^{2}}=T \frac{\partial^{2} y}{\partial z^{2}}-s_{y} \frac{\partial^{4} y}{\partial z^{4}}-2 \mu_{y} \lambda y \frac{\partial y}{\partial t} \\
& +k \rho_{s} \frac{\partial x}{\partial t}, \quad 2-12 b
\end{aligned}
$$

where $\mu=\mu_{w}+\mu_{s}+K \mu_{n}$, $\mu_{w}$ being the wire mass per unit length, T the tension in the wire, and $\lambda=\frac{w K^{\prime}{ }^{\prime} n}{2 \mu}$. $S$ is the stiffness constant given by $S=Y_{A R}{ }^{2}$ where $\mathrm{Y}$ is Young's modulus, A is the cross-sectional area of the wire, and $R$ is the radius of gyration of a cross-section about an axis through its centroid and in the plane of the cross-section, perpendicular to the plane of oscillation. (24) The constants $\mu, \lambda$, and $S$ are in general different for the two axis directions. On1y in the case of a wire with 
circular sections are they identical. In the solutions obtained here it will be assumed that for a given axis direction $\mu, \lambda, S$, and $T$ are all uniform along the length of the wire.

6. Motion of the Wire in a Non-Rotating Coordinate Frame with Zero Circulation

We now search for solutions to Eq. 2-12a and 2-12b with the circulation zero. We assume a rigid mounting for the wire. Then the boundary conditions are that the displacement and slope of the wire at the end points of the wire be zero. We look for solutions of the form

$$
\begin{aligned}
& x_{0}(z, t)=\operatorname{Re}\left(f_{0}(z) e^{i n x_{0}^{x} t}\right) \\
& y_{0}(z, t)=\operatorname{Re}\left(g_{0}(z) e^{i n y_{0} t}\right),
\end{aligned}
$$

where the subscript o denotes solutions to the equations of motion in the absence of circulation and rotation. With the origin at the midpoint of the wire the boundary conditions are, for a wire of length L

$$
\begin{array}{lll}
f_{0}\left( \pm \frac{L}{2}\right)=0, & f_{0}^{\prime}\left( \pm \frac{L}{2}\right)=0, & 2-14 a \\
g_{0}\left( \pm \frac{L}{2}\right)=0, & g_{0}^{\prime}\left( \pm \frac{L}{2}\right)=0 . & 2-14 b
\end{array}
$$

The solutions to the $x$ equation for the amplitude are of the form 


$$
\mathrm{f}_{0}(\mathrm{z})=\mathrm{A} \cos \left(\frac{\mathrm{m}_{1} \pi \mathrm{z}}{\mathrm{L}}\right)+B \cosh \left(\frac{\mathrm{m}_{2} 2^{\pi \mathrm{z}}}{\mathrm{L}}\right) \quad 2-15 \mathrm{a}
$$

for the even modes and

$$
f_{0}(z)=C \sin \left(\frac{m_{1} \pi z}{L}\right)+D \sinh \left(\frac{{ }^{m} 2^{\pi z}}{L}\right)
$$

for the odd modes. (25) The complex frequency $n_{0}^{x}$ has the form

$$
n_{0}^{x}=\sqrt{\omega_{0}^{x^{2}}-\lambda_{x}^{2}}+i \lambda_{x}
$$

and the allowed frequencles $\omega_{0}$ are found from the expression

$$
\omega_{0}^{x}=\frac{m_{1} \pi}{L} \sqrt{\left(T / \mu_{x}\right)\left[1+\left(\frac{m_{1} \pi}{L}\right)^{2} \frac{S}{T}\right]},
$$

where $m_{1}$ is found by solving the transcendental equations obtafned from application of the boundary conditions

$$
\begin{aligned}
& \tan \left(\frac{\mathrm{m}_{1}}{2}\right)=\mp\left(\sqrt{1+\left(\frac{\mathrm{L}}{\mathrm{m}_{1} \pi}\right)^{2} T / S_{y}}\right) \pm 1 \\
& \times \tanh \left(\frac{\mathrm{m}_{1}}{2} \sqrt{1+\left(\frac{\mathrm{L}}{\mathrm{m}_{1} \pi}\right)^{2} T / S_{x}}\right)
\end{aligned}
$$

for the even and odd modes respectively.

For small $\mathrm{S}_{\mathrm{x}}$, the allowed frequencies of a flexible string are recovered:

$$
\omega_{0}^{\mathrm{x}}=\frac{\mathrm{m}_{1}{ }^{\pi}}{\mathrm{L}} \sqrt{\mathrm{T} / \mu_{\mathrm{x}}}, \quad \frac{\mathrm{S}_{\mathrm{x}}}{\mathrm{T}}\left(\frac{{ }^{{ }_{1}}{ }^{\pi}}{\mathrm{L}}\right)^{2} \ll 1 . \quad 2-19
$$

In this limit $m_{1}=1,2 \ldots$ Also in this limit the 
corresponding amplitude functions are those obtained from Eq. 2-12

$$
\begin{array}{ll}
f_{0}(z)=A \cos \left(\frac{m_{1} \pi}{L} z\right) & 2-20 a \\
f_{0}(z)=A \sin \left(\frac{m_{1}}{L} z\right) & 2-20 b
\end{array}
$$

for the even and odd modes.

An expansion of Eq. 2-17 in the parameter $\frac{2}{L} \sqrt{S_{x} / T}$ shows that for low modes the wire frequency is that of a flexible string of lengch $L^{*}=\mathrm{L}-2 \sqrt{\mathrm{S}_{\mathrm{X}} / \mathrm{T}}$. We find from Eq. 2-12 in this limit that a wire with stiffness $S_{x}$ can be described by Eqs. 2-19 and 2-20 If $L$ is replaced by $L^{*}$.

The actual angular frequency of the normal modes is given by

$$
\operatorname{Ren}_{0}^{x}=\sqrt{\omega_{0}^{x^{2}}-\lambda_{x}^{2}}
$$

This frequency depends on the decay constant $\lambda$, which itself depends on the frequency. For $\lambda_{x}$ small compared to $\omega_{0}^{\mathrm{x}}$ we have

$$
\operatorname{Ren}{ }_{0}^{x}=\omega_{0}^{x}\left(1-\lambda_{x}^{2} / 2 \omega_{0}^{x 2}\right) .
$$

The correction to the angular frequency due to the damping is thus in our work of maximum value 


$$
\lambda_{x}^{2} / 2 \omega_{0}^{x^{2}}=\left(\frac{K^{\prime} \mu}{4 \mu}\right)^{2}=10^{-6} .
$$

As we shall see, the corrections to the frequency introduced by rotation and circulation are of order $10^{-3} \omega_{0}$. Thus, we may ignore the effect of the damping constant on the frequency. The only importanteffect of the damping term is to damp the amplitude of the modes exponentially.

The solutions for Eq. $2-12 b$ are of the same form as those just obtained for Eq. 2-12a. In general, since $\lambda_{y}, s_{y}$, and $\mu_{y}$ differ from $\lambda_{x}, S_{x}$, and $H_{x}$, the $p l a n e-p o l a r i z e d$ modes in the $y-z$ plane will have different frequencies than those in the $x-z$ plane. The functions $g_{0}(z)$ will then also be different from $f_{0}(z)$, except in the $L *$ limit.

It should be noted that the boundary conditions applied depend upon the physical arrangements of the wire support. If the supports were not perfectly rigld, for example, the boundary conditions would take a different form. Every effort was made in the experiment to achieve conditions in which the boundary conditions, Eqs. 2-14a and 2-14b, apply, and any deviation from them will not be treated in this analysis. 
7. The Motion of the Wire for Non-Zero Circula-

\section{tion and in Rotation}

When circulation is present around the wire the equations of motion are coupled by the Magnus force. In addition, when the entire system is in rotation, the equations will be coupled by additional terms due to the rotation. When the system is in rotation, the equations of motion must be transformed to the rotating system in which the transverse oscillations take place and are observed. To make this transformation we make use of a result of classical mechanics. (26) If we have two coordinate systems whose origins are separated by some distance $\vec{h}$ and one system is rotating with respect to the other, then the velocity and acceleration of a given point in space, as described in the two systems, are related by

$$
\begin{aligned}
& \left(\frac{d \vec{r}^{\prime}}{d t}\right)_{f}=\left(\frac{d \vec{r}}{d t}\right)_{r}+\vec{\omega}_{r} \times \vec{r}+\left(\frac{d \vec{h}}{d t}\right)_{f}, \\
& \left(\frac{d^{2} \vec{r}^{\prime}}{d t^{2}}\right)_{f}=\left(\frac{d^{2} \vec{r}}{d t}\right)_{r}+\vec{\omega}_{r} \times\left(\vec{\omega}_{r} \times \vec{r}\right) \\
& \quad+2 \vec{\omega}_{r} \times\left(\frac{d \vec{r}}{d t}\right)_{r}+\left(\frac{d \vec{\omega}}{d t}\right)_{f} \times \vec{r}+\left(\frac{d^{2} \vec{h}}{d t^{2}}\right)_{f}, 2-23 b
\end{aligned}
$$

where $\vec{r} \cdot=\vec{r}+\vec{h}$, and the unprimed system is assumed rotating with respect to the primed system. The 
derivative $(\mathrm{d} \vec{r} / / d t)_{f}$ is to be evaluated in the fixed frame and the derivative $(\mathrm{d} \vec{r} / d t)_{r}$ is to be evaluated in the rotating frame. We further assume that if there is any displacement, the inner and outer cylinders are both displaced an equal distance from the axis of rotation, so that the cylinders remain co-axial but rotating about an axis different from their common axis. We will show that the effect of this displacement is not important, and we will not include terms in $\vec{h}$ in the transformed equations. Finally, we assume that the wire and the axis of rotation are parallei and that the wire lies along the $z$-axis in the rotatiug frame. Then, with these assumptions, and using the transformation equations to transform Eqs, 2-12a and $2-12 b$, we obtain

$$
\begin{aligned}
& \mu_{x} \frac{\partial^{2} x}{\partial t^{2}}=T \frac{\partial^{2} x}{\partial z^{2}}-S_{x} \frac{\partial^{4} x}{\partial z^{4}}-2 \mu_{x}{ }^{\lambda} x \frac{\partial x}{\partial t} \\
& +\left(\mu_{x}{ }^{\omega}{ }_{r}^{2}-\rho_{s}{ }^{k \omega_{r}}\right) x+\left(2 \mu_{x}{ }^{\omega} r-\rho_{s} k\right) \frac{\partial y}{\partial t} \\
& +\left(2 \mu_{x}{ }^{\lambda} x^{\omega s} r+\mu_{x}{ }^{\omega}{ }_{r}\right) y, \quad 2-24 a
\end{aligned}
$$




$$
\begin{aligned}
\mu_{y} \frac{\partial^{2} y}{\partial t^{2}} & =T \frac{\partial^{2} y}{\partial z^{2}}-S_{y} \frac{\partial^{4} y}{\partial z^{4}}-2 \mu_{y} \lambda_{y} \frac{\partial y}{\partial t} \\
& +\left(\mu_{y} \omega_{r}^{2}-\rho s^{k \omega_{r}}\right) y-\left(2 \mu_{y} \omega_{r}-0 s^{k}\right) \frac{\partial x}{\partial t} \\
& -\left(2 \mu_{y}{ }^{\lambda} \omega_{r}+\mu_{y} \dot{\omega}_{r}\right) x .
\end{aligned}
$$

We note that by using the purely kinematic transformation of Eqs. 2-23a and 2-23b to obtain the equations of motion of the wire when the system is in rotation we are ignoring hydrodynamical effects which arise because of the rotational motion of the fluid.

With respect to the superfluid, we expect no additional effects due to rotation. The boundary conditions applied in computing the force acting on the wire due to the superfluid circulation are unaltered by the rotation of the container and wire. The circulation which is determined by these measurements is the circulation as defined in the nonrotating system.

In the case of the normal fluid, the rotation of the system brings it into rigid-body flow. As a consequence, the normal fluid forces which act on the wire are expected to be altered from those considered earlier. Experimentally no significant 
change in the damping is seen, suggesting that no significant change in the normal fluid forces a1ready calculated is brought about by the rotation. The radial pressure gradient in the normal fluid brought about by the rotation gives rise to a radial buoyant force which acts on the wire as it vibrates and is displaced from the cylinder axis. Also the rotation of the normal fluid in rigid body flow gives rise to a uniform circulation density in the fluid, $2 w_{r}$. These effects have not been included in this treatment.

We note that the circulation mas vary along the length of the wire. For example if a vortex line terminates on the wire the circulation around the wire will change by an amount equal to the circulation of that vortex. Because of effects such as this we will treat the circulation $k$ as a function of $z$, the distance along the wire. We also note that an attached vortex 11 ne will alter the tension in the wire but by a negligible amount. In addition an attached vortex line would alter the assumed two-dimensional superfluid flow pattern. This effect is not consldered.

When there is circulation around the wire there is present a hydrodynamic tension which acts on the 
wire and arises from the deformation of the irrotational flow brought about by the vibrating wire. (27) This hydrodynamic tension is of the form

$$
T_{H}=\left(\rho_{s} \kappa^{2} / 4 \pi\right)[\ln (2 / k a)-\gamma] .2-25
$$

Here $k$ is the circulation, $p_{s}$ is the superfluid density, a the wire radius, $k$ the wave number of the wire oscillation, and $\gamma=0.577 \ldots$ This hydrodynamic tension is to be compared with $T$, the wire tension. For the low modes of the wire oscillation we have

$$
T_{H} / T=\frac{\rho_{S} k^{2} \ln (1.12 / k a)}{4 L^{2} \omega^{2} \mu} \ll 1 .
$$

A further point to be made is that in nonsteady rotation, if the accelerations are large, deviations from rigid-body flow of the normal fluid become possible. However, the longest time constant associated with changes in the viscous flow is 2 seconds at $1.3 \mathrm{~K}$. On the other hand, the accelerations used in this experiment never exceeded $10^{-4} \mathrm{rad} / \mathrm{sec}^{2}$, and the period of the wire vibration was typically of order $10^{-3} \mathrm{sec}$. Consequently, any deviations from rigid-body flow were unimportant. Further, we note that 
both over the period of a wire vibration, and over the time in which a measurement was made, approximately $1 \mathrm{sec}$, accelerations of the order of $10^{-4}$ $\mathrm{rad} / \mathrm{sec}^{2}$ bring about neg1igibly small changes in the angular velocity. Finally, a term proportional to the angular acceleration in the equations of motion has a negligible effect upon a frequency of the normal modes for the angular accelerations used in this experiment. Because of these considerations, we w111 Ignore terms proportional to the angular acceleration.

In the transformation equations we have included terms in $\vec{h}$, the separation of the origins of the coordinate systems. The appearance of these terms in the equations of motion would have no effect on the allowed frequencies of vibration of the wire or the wire's velocity. The net effect is to introduce a constant shift in the amplitude equations. Since this shift has no effect on the observations, these terms in $\vec{h}$ have been dropped from the equations of motion. However, there would be hydrodynamic effects if the rotation axis of the fluid and the wire were not coincident. For example, the pressure gradient in the normal fluid mentioned before would 
give rise to a force per unit length of the form F $\alpha \rho{ }_{n}{ }^{2}{ }^{2} h$. This force would also not effect the frequencies and has been ignored. Also, every attempt was made to insure that the rotation axis and wire axis were coincident, and direct measurement showed they differed by less than $0.2 \mathrm{~mm}$. We now seek solutions to Eqs. $2-24 \mathrm{a}$ and $2-24 \mathrm{~b}$. We assume solutions of the form

$$
\begin{array}{ll}
x_{m}(z, t)=\operatorname{Re}\left(f_{m}(z) e^{i n m t}\right), & 2-26 a \\
y_{m}(z, t)=\operatorname{Re}\left(g_{m}(z) e^{i \eta_{m} t}\right) & 2-26 b
\end{array}
$$

with boundary conditions, as before,

$$
\begin{array}{ll}
f_{m}\left( \pm \frac{L}{2}\right)=0, & f_{m}^{\prime}\left( \pm \frac{L}{2}\right)=0, \\
g_{m}\left( \pm \frac{L}{2}\right)=0, g_{m}^{\prime}\left( \pm \frac{L}{2}\right)=0 . & 27 a
\end{array}
$$

We note that the solutions $f_{\text {mo }}(z)$ and $g_{\text {mo }}(z)$ obtained in the case of no rotation and no circulation, when normalized, both form complete orthonormal sets of functions. Any function which satisfies the same boundary conditions as these functions and has continuous first and second derivatives and piece-wise continuous third and fourth derivatives may be expanded in terms of these functions in an absolutely and uniformly 
convergent series. (28) Thus $f_{m}(z)$ and $g_{m}(z)$ may both be expanded in terms of either one of these sets of functions. Thus we may write

$$
\begin{aligned}
& f_{m}(z)=\sum_{n=1}^{\infty} \alpha_{m n} f_{n o}(z) \\
& g_{m}(z)=\sum_{n=1}^{\infty} \beta_{m n} g_{n o}(z),
\end{aligned}
$$

where the coefficients $\alpha_{m n}$ and $\beta_{m n}$ are in general complex. The numbers $m$ and $n$ are counting indices indicating the $\mathrm{m}^{\mathrm{th}}$ and $\mathrm{n}^{\mathrm{th}}$ allowed set of values of $m_{1}$ and $m_{2}$. Substituting these equations into the equations of motion we obtain

$$
\begin{aligned}
\sum_{n=1}^{\infty} \alpha_{m n} & {\left[2 i \lambda_{x}\left(n_{m}-n_{n o}^{x}\right)-\left(n_{m}^{2}-n_{n o}^{x^{2}}\right)\right.} \\
& \left.+\left(\frac{\rho s^{k}}{\mu_{x}} \omega_{r}-\omega_{r}^{2}\right)\right] f_{n o} \\
& +\sum_{n=1}^{\infty} B_{m n}\left[\left(\frac{\rho s^{k}}{\mu_{x}}-29 a\right.\right.
\end{aligned}
$$

and

$$
\begin{aligned}
\sum_{n=1}^{\infty} \beta_{m n}\left[2 i \lambda_{y}\left(n_{m}-n_{n o}^{y}\right)-\left(n_{m}^{2}-n_{n o}^{y^{2}}\right)\right. & 2-2 \\
& \left.-\left(\omega_{r}^{2}-\frac{\rho_{s}{ }^{k}}{\mu_{y}} \omega_{r}\right)\right] g_{n o} \\
& -\sum_{n=1}^{\infty} a_{m n}\left[\left(\frac{{ }_{s}{ }^{k}}{\mu_{y}}-2 \omega_{r}\right) i n_{m}-2 \lambda_{y}{ }^{\omega_{r}}\right] f_{n o}=0 .
\end{aligned}
$$


Multiplying Eq. 2-29a by $f_{m o}(z)$ and Eq. $2-29 b$ by $g_{m o}(z)$ and integrating over the allowed range of $z$ using the orthonormality conditions, we obtain

$$
\begin{aligned}
& \alpha_{m m}\left[2 i \lambda_{x}\left(n_{m}-n_{m o}^{x}\right)-\left(n_{m}^{2}-n_{m o}^{x^{2}}\right)-\omega_{r}^{2}\right] \\
& +\sum_{n=1}^{\infty} \alpha_{m n} \frac{p_{s}{ }^{\omega} x}{\mu_{x}} k_{m n}^{\prime} \\
& +\sum_{n=1}^{\infty} \beta_{m n}\left[\left(2 \lambda x^{\omega} r-2 i \omega_{r}{ }^{n}\right) I_{m n}\right. \\
& \left.+\frac{\rho_{s}}{\mu_{x}} k_{m n}^{\prime \prime} i n_{m}\right]=0
\end{aligned}
$$

and

$$
\begin{aligned}
& B_{m m}\left[21 \lambda_{y}\left(n_{m}-n_{m o}^{y}\right)-\left(n_{m}^{2}-n_{m o}^{y^{2}}\right)-\omega_{r}^{2}\right] \\
& +\sum_{n=1}^{\infty} B_{m n} \frac{\rho s^{\omega} r}{{ }_{y}} k_{m n}^{\prime \prime \prime} \\
& +\sum_{n=1}^{\infty} a_{m n}\left[\left(2 i \omega{ }_{r}{ }^{n} m+2 \lambda_{y}{ }^{\omega}{ }_{r}\right) I_{m n}^{\prime}\right. \\
& \left.-\frac{P_{\mathrm{s}}}{\mu_{\mathrm{y}}} \mathrm{k}_{\mathrm{mn}}^{\cdots,} i n_{\mathrm{m}}\right]=0,
\end{aligned}
$$

where

$$
\begin{aligned}
& K_{m m}^{\prime}=\int_{-L / 2}^{L / 2} k(z) f_{m o}(z) f_{n o}(z) d z, \\
& k_{m n}^{\prime \prime}=k_{n m}^{\prime \prime \prime}=\int_{-L / 2}^{L / 2} k(z) f_{m o}(z) g_{n o}(z) d z, \\
& k_{m n}^{\prime \prime \prime}=\int_{-L / 2}^{L / 2} k(z) g_{m o}(z) g_{n o}(z) d z,
\end{aligned}
$$

and 
$\mathrm{L} / 2$

$$
I_{m n}=I_{n m l}^{\prime}=\int f_{n o}(z) g_{m o}(z) d z . \quad 2-31 d
$$

If the wire is symmetric and $k(z)$ is uniform along the length of the wire then only the integrals for $n=m$ survive in the expansions in Eqs. 2-30a and $2-30 b$. The equations then are very simple and determine directly the ratios $\alpha_{\mathrm{mm}} / B_{\mathrm{mm}}$ and the frequencies for this system. The solutions are linear combinations of the plane-polarized modes found ear1ier and have circular polarization. We sha11 assume in the more general case of the asymmetric wire with non-uniform circulation that only those eigenfunctions for which $n=m$ make a significant contribution to the motion. This assumption implies that for $n \neq m$

$$
\left|\frac{\mathrm{mm}}{\alpha_{\mathrm{mm}}}\right| \ll 1 \quad 2-32 \mathrm{a}
$$

and

$$
\left|\frac{B_{m n}}{B_{m m}}\right| \ll 1 .
$$

We note

$$
k_{m m}^{\prime \prime}=k_{m m}^{\prime \prime \prime}=\int_{-L / 2}^{L / 2} \kappa(z) f_{m o}(z) g_{m o} d z .
$$

We will call this quantity the apparent circulation, $\bar{\kappa}_{\mathrm{m}}$. 
The integral

$$
I_{m m}=I_{m m}^{\prime}=\int_{-L / 2}^{L / 2} E_{m o}(z) g_{m o}(z) d z
$$

may be evaluated using Eqs. $2-15$ a and $2-15 b$ for $f_{\text {mo }}(z)$ and similar equations for $g_{\text {mo }}(z)$. Direct evaluation shows that the integral differs from unity in the lower modes by a term which for this experiment is of the order $10^{-6}$. Thus, to a good approximation, we have $I_{m m}=I_{m m}^{\prime}=1$.

Then, letting $\mathrm{k}_{\mathrm{mm}}^{\prime}=\bar{\kappa}_{\mathrm{mx}}$ and $\mathrm{k}_{\mathrm{mm}}^{\prime \prime \prime}=\bar{k}_{\mathrm{my}}$ we obtain

$$
\begin{aligned}
& \alpha_{m m}\left[2 i \lambda_{x}\left(n_{m}-n_{m o}^{x}\right)-\left(n_{m}^{2}-n_{m o}^{x^{2}}\right)-\left(\omega_{r}^{2}-\frac{\rho_{s} \bar{k}_{m x}}{\mu_{x}} \omega_{r}\right)\right] \quad 2-34 a \\
& \left.+\beta_{m m}\left[\left(\frac{\rho_{s} \bar{k}_{m}}{\mu_{x}}-2 \omega_{r}\right) i n_{m}+2 \lambda_{x} \omega_{r}\right)\right]=0
\end{aligned}
$$$$
\beta_{m m}\left[2 i \lambda_{y}\left(n_{m}-n_{m o}^{y}\right)-\left(n_{m}^{2}-n_{m o}^{y^{2}}\right)-\left(\omega_{r}^{2}-\frac{\rho_{s} \bar{k}_{m y}}{H_{y}} w_{r}\right)\right] 2-34 b
$$$$
-\alpha_{m m}\left[\left(\frac{\rho s \bar{K}_{m}}{\mu_{y}}-2 \omega_{r}\right) i n_{m}-2 \lambda_{y} \omega_{r}\right]=0 .
$$

To obtain solutions to these equations we note that the determinant of the coefficients $\alpha_{\mathrm{mm}}$ and $B_{\mathrm{mm}}$ must vanish. We now use our previous observation that terms proportional to the damping constant have little effect on the frequencles, entering into the 
problem primarily in yielding an exponential damping to the amplitudes. We ignore these terms in calculating the frequencies.

The determinant of the coefficlents of $\alpha_{\mathrm{mm}}$ and $\beta_{m m}$ may then be expanded, yielding

$$
\left(n_{m}^{2}-\xi_{x} \xi_{y}\right)^{2}-n_{m}^{2}\left(x_{x} x_{y}+\left(\xi_{x}-\xi_{y}\right)^{2}\right)=0 \quad 2-35
$$

where

$$
\begin{aligned}
& \xi_{x}=n_{m 0}^{x}\left[1+\frac{\omega_{r}\left(\omega_{r}-\frac{\rho_{s} \bar{k}_{m x}}{\mu_{x}}\right)}{n_{m o}^{x^{2}}}\right]^{1 / 2}, \quad 2-36 \\
& x_{x}=\frac{\rho_{s} \bar{K}_{m}}{{ }_{x}}-2 \omega_{r},
\end{aligned}
$$

with similar expressions for $\varepsilon_{y}$ and $x_{y}$. We note that to a good approximation

$$
\begin{aligned}
& \xi_{x}=n_{m o}^{x}, \\
& \xi_{y}=n_{m o}^{y},
\end{aligned}
$$

and

$$
x_{x} x_{y}=\left(\frac{\rho_{s} \bar{k}_{m}}{\left(\mu_{x} \mu_{y}\right)^{1 / 2}}-2_{{ }_{r}}\right)^{2} . \quad 2-39
$$

With these approximations we then obtain two solutions for the angular frequencies of the $m^{\text {th }}$ pair of normal modes: 


$$
\omega_{m}^{ \pm}=\omega_{m o} \pm \frac{1}{2} \sqrt{\left(\Delta \omega_{m o}\right)^{2}+\left(\Delta \omega_{m k}-\Delta \omega_{r}\right)^{2}} \quad 2-40
$$

where

$$
\begin{array}{ll}
\omega_{m o}=\sqrt{\frac{\omega_{m o}^{x^{2}}+\omega_{m o}^{y^{2}}}{2}}, & 2-41 a \\
\Delta \omega_{m o}=\omega_{m o}^{x}-\omega_{m o}^{y}, & 2-41 b \\
\Delta \omega_{m k}=\frac{\rho_{s^{k} m}}{\sqrt{\mu_{x}^{\mu} y}}, & 2-41 c
\end{array}
$$

and

$$
\Delta w_{r}=2 w_{r} \text {. }
$$

The total frequency splitting between the two modes for a given $m$ is then

$$
\Delta w_{m t}=\sqrt{\left(\Delta w_{m o}\right)^{2}+\left(\Delta w_{m k}-\Delta w_{r}\right)^{2}} \cdot 2-42
$$

In obtaining these solutions we assume that $\Delta \omega_{\text {mo }}$, $\Delta \omega_{m K}$, and $\Delta \omega_{r}$ are al1 much less than $\omega_{m o}^{x}, \omega_{m o}^{y}$, and ${ }^{\omega}$ mo ${ }^{*}$

$$
\text { For the symmetric wire } \Delta \omega_{\text {mo }}=0 \text { and the equations }
$$
reduce to

$$
\omega_{\mathrm{m}}^{ \pm}=\omega_{\mathrm{mo}} \pm \frac{1}{2}\left(\Delta_{\mathrm{m}}-\Delta \omega_{\mathrm{r}}\right),
$$

with

$$
\Delta \omega_{\mathrm{mK}}=\frac{\rho \mathrm{s}^{\bar{k}} \mathrm{~m}}{\mu} .
$$


If $x(z)$ is uniform along the wire then, for the symmetric wire, the apparent circulation is equal to the true circulation around the vire. For the asymmetric wire with unfform circulation

$$
\bar{k}_{m}=k \int_{-L / 2}^{L / 2} f_{m o}(z) g_{m o}(z) d z,
$$

and $\bar{k}_{m}$ differs from $k$ by the amount that the integral differs from unity, approximately $10^{-6}$ in the lower modes.

The calculated mass per unit length $\mu$ and the quantity $\sqrt{{ }^{\mu} x^{\mu} y}$ differ because of differences in the contribution of the wire's ellipticity to the hydrodynamic mass per unit length of the wire. However, since the wires are twisted, irregularities in them tend to cancel out. Under these conditions we expect negligible differences between $\sqrt{\mu^{\mu} y}$ and H.

8. The Norma1-Mode Equations

Having found the frequencies of the wire's motion, we can now find the ratios of the coefficients in Eqs. $2-34 \mathrm{a}$ and $2-34 \mathrm{~b}$ by substituting the frequencies. Making this substitution we arrive at the expression 


$$
B_{\mathrm{mm}}^{ \pm}=\mp i \mathrm{R}_{\mathrm{m}}^{ \pm 1} \alpha_{\mathrm{mm}}^{ \pm},
$$

where

$$
R_{m}, \frac{\Delta \omega_{m t}+\Delta \omega_{m o}}{\Delta \omega_{m k}-\Delta \omega_{r}} .
$$

Note that for a symmetric wire $\Delta \omega_{m o}=0$ and $\left|R_{m}\right|=1$. We now set $\alpha_{\mathrm{mm}}^{ \pm}=A_{m}^{ \pm} e^{i \phi} \frac{ \pm}{m}$, where $A_{m}^{ \pm}$and $\phi_{m}^{ \pm}$are real. Then the solutions to Eq. $2-24 \mathrm{a}$ and $2-24 \mathrm{~b}$ may be written

$$
\begin{aligned}
x_{m}^{ \pm}(z, t) & =\operatorname{Re}\left[A_{m}^{ \pm} f_{m o}(z) e^{i\left(\omega_{m}^{ \pm} t+\phi_{m}^{ \pm}\right)}\right] e^{-\lambda_{m}^{ \pm} t} 2-48 a \\
& =A_{m}^{ \pm} f_{m o}(z) \cos \left(\omega_{m}^{ \pm} t+\phi_{m}^{ \pm}\right) e^{-\lambda ~_{m}^{t}} \quad 2-49 a
\end{aligned}
$$

and

$$
\begin{aligned}
y_{m}^{ \pm}(z, t) & =\operatorname{Re}\left[\overline{+}_{\mathrm{m}} A_{\mathrm{m}}^{ \pm} g_{\mathrm{mo}}(\mathrm{z}) \mathrm{e}^{i\left(\omega_{\mathrm{m}}^{ \pm} \mathrm{t}+\phi_{\mathrm{m}}^{ \pm}\right)}\right] \mathrm{e}^{-\lambda_{\mathrm{m}}^{ \pm} \mathrm{t}} 2-48 \mathrm{~b} \\
& = \pm \mathrm{A}_{\mathrm{m}}^{ \pm} \mathrm{R}_{\mathrm{m}}^{ \pm 1} \mathrm{~g}_{\mathrm{mo}}(\mathrm{z}) \sin \left(\omega_{\mathrm{m}}^{ \pm} \mathrm{t}+\phi_{\mathrm{m}}^{ \pm}\right) \mathrm{e}^{-\lambda_{\mathrm{m}}^{ \pm}} \cdot \quad 2-49 \mathrm{~b}
\end{aligned}
$$

To study the behavior of the normal modes let

$$
\psi_{m}^{ \pm}(z, t)=x_{m}^{ \pm}(z, t)+1 y_{m}^{ \pm}(z, t) .
$$

We further assume $f_{\text {mo }}(z) \approx g_{\text {mo }}(z)$. These functions differ at any given $z$ in the lower modes by amounts of order $\Delta \omega_{\text {mo }} / \omega_{\text {mo }}$, and amplitude effects of this size are not of interest. Then, forming the modes, we have 


$$
\begin{aligned}
& \psi_{\mathrm{m}}^{ \pm}(z, t)=\mathrm{A}_{\mathrm{m}}^{ \pm} \mathrm{E}_{\mathrm{mo}}(\mathrm{z}) \mathrm{e}^{-\lambda \mathrm{m}^{ \pm} \mathrm{t}} \\
& \times\left[\cos \left(\omega_{\mathrm{m}}^{ \pm} \mathrm{t}+\phi_{\mathrm{m}}^{ \pm}\right) \pm i \mathrm{R}_{\mathrm{m}}^{1} \sin \left(\omega_{\mathrm{m}}^{ \pm} \mathrm{t}+\phi_{\mathrm{m}}^{ \pm}\right)\right], \quad 2-50 \mathrm{a}
\end{aligned}
$$

which can be rewritten

$$
\begin{aligned}
& \psi_{\mathrm{II}}^{\prime}(\mathrm{z}, \mathrm{t})=\mathrm{A}_{\mathrm{m}}^{+} \mathrm{f} \text { mo } \\
& \times\left[\cos ^{2}\left(\omega_{\mathrm{m}}^{ \pm} \mathrm{t}+\phi_{\mathrm{m}}^{ \pm}\right)+\mathrm{R}_{\mathrm{m}}^{ \pm 2} \mathrm{sin}^{2}\left(\omega_{\mathrm{m}}^{ \pm} \mathrm{t}+\phi_{\mathrm{m}}^{ \pm}\right)\right]^{1 / 2} \pm 1 \theta_{\mathrm{m}}^{ \pm},
\end{aligned}
$$

where

$$
\tan \theta_{m}^{ \pm}=R_{m}^{ \pm 1} \tan \left(\omega_{m}^{ \pm} t+\phi_{m}^{ \pm}\right)
$$

For the symmetric wire $\left|R_{\mathrm{m}}\right|=1$ and $\Delta \omega_{\text {mo }}=0$, so that the normal modes become

$$
\begin{aligned}
& \Psi_{m}^{\dagger}(z, t)=A_{m}^{ \pm} f_{m o}(z) e^{-\lambda_{m}^{ \pm} t} e^{ \pm 1 \phi_{m}^{ \pm}} \\
& \quad x e^{ \pm i\left(s g n R_{m}\right)\left(\omega_{m o} \pm \frac{1}{2}\left(\Delta \omega_{m K}-\Delta \omega_{r}\right)\right) t} \cdot \quad 2-52
\end{aligned}
$$

These functions describe the two circularly polarized modes of the symmetric wire when the apparatus is in rotation and in the presence of circulation. For each integer m there are two modes, with angular frequencies differing by $\Delta w_{\text {mk }}-\Delta \omega_{r}$. The sign of this splitting depends upon the relative signs and magnitudes of the quantities $\Delta \omega_{\mathrm{m}}$ and $\Delta \omega_{\mathrm{r}}{ }^{\text {. The mode }}$ $\gamma_{m}^{+}$rotates counter-clockwise if $\mathrm{sgnR}_{\mathrm{m}}$ is positive. 
In the particular case that the method of excitation results in the two modes given in Eq. 2-52 being excited with equal amplitude and phase the resulting motion, for the case of the symmetric wire, is given by

$$
\begin{aligned}
\psi_{m}^{-}+ & \psi_{m}^{+}=2 A_{m} f_{m o}(z) e^{-\lambda} t \\
& \times \cos \left(\omega_{m o} t+\phi_{m}\right) e^{(i / 2)\left(\Delta \omega_{m k}-\Delta \omega_{r}\right) t} \cdot 2-53
\end{aligned}
$$

If the circulation is uniform along the length of the wire $\Delta \omega_{m K}=\Delta w_{k}$ for these modes. The wire thus oscillates in a plane which rotates at a rate $\left(\Delta \omega_{K}-\Delta \omega_{r}\right) / 2$. The rotation is in the counterclockwise direction if $\left(\Delta \omega_{K}-\Delta \omega_{r}\right)>0$ and clockwise if $\left(\Delta \omega_{k}-\Delta \omega_{r}\right)<0$.

In the general case of the asymmetric wire similar considerations hold. In this case each of the $\mathrm{m}^{\text {th }}$ pair of normal modes is elliptically polarized, with eccentricity $e_{m}=\left(1-1 / \mathrm{R}_{\mathrm{m}}^{2}\right)^{1 / 2}$. These modes differ in frequency by

$$
\Delta \omega_{m t}=\sqrt{\left(\Delta \omega_{m o}\right)^{2}+\left(\Delta \omega_{m k}-\Delta \omega_{r}\right)^{2}} \cdot 2-54
$$

The higher-frequency mode rotates in the direction given by the sign of the quantity $\Delta \omega_{m_{k}}-\Delta_{k}{ }^{*}$ The 
rotation has a positive (counter-clockwise) sense if this sign is positive and a negative sense if this sign is negative.

\section{Observation of the Motion}

The wire motion was observed by exciting it in the presence of a transverse magnetic field whose direction was fixed in the rotating frame. The amplitudes and phases of the various modes excited were determined by the conditions under which the modes were initially excited and the current pulse used for excitation.

We assume that the magnetic field is oriented at an angle of $-\pi / 4$ radians with respect to the positive $x$-axis. We also assume that the magnetic field is unfform over the length of the wire.

The pulse used to excite the wire's motion was a sinusoidal wave train at the fundamental frequency of the wire's motion, which we shall label $w_{1}$. The force per unit length acting on the wire while it is being excited may then be written

$$
\begin{array}{ll}
\overrightarrow{\mathrm{F}}(t) / L=I_{0} B \sin \left(\omega_{1}\left(t-t^{\prime}\right)\right)\left(\frac{\hat{i}+\hat{i}}{2}\right) & t^{\prime} \leq t \leq 0,2-55 a \\
\vec{F}(t) / L=0 & t<t^{\prime}, 2-55 b \\
& t>0,
\end{array}
$$


where $\hat{i}$ and $\hat{j}$ are unit vectors in the $x$ and $y$ directions, respectively. The wire is thus driven for a length of time equal to $\left|t^{\prime}\right|$.

\section{Model Calculation}

To determine the wire's response to this drive we will consider the case of a flexible, symmetric wire of length $L^{*}=L-2 \sqrt{\mathrm{S} / \mathrm{T}}$. Then if the system is in rotation and if we assume that uniform circulation is present, the equations of motion may be written as

$$
\mu \frac{\partial^{2} \psi}{\partial t^{2}}=T \frac{\partial^{2} \psi}{\partial z^{2}}-\left(2 \lambda_{\mu-1}\left(\rho_{S} k-2 \mu \omega r\right)\right) \frac{\partial \psi}{\partial t}+f(t), 2-56
$$

where

$$
\Psi(z, t)=x(z, t)+i y(z, t), \quad 2-57
$$

and

$$
f(t)=I_{0} B \sin \left(\omega_{1} t\right) e^{i \pi / 4} . \quad 2-58
$$

Equation 2-46 is obtained from Eqs. 2-22a and $2-22 b$ using the assumption that the wire is symmetric. Furthermore, we have ignored the stiffness, assuming that its effects are accounted for by the use of the length $L^{*}$. Finally, small terms in Eqs, 2-22a and 2-22b found previous1y not to effect the frequencies have been ignored. 
We note that this system consists of a vibrating wire being driven resonantly in its fundamental mode by the force $f(t)$. Because of this method of excitation only the fundamental mode is significantly excited, and only the fundamental mode will be considered in our subsequent discussion of the motion. Furthermore, the oscillations of the exciter wire are observed by means of the emf which is generated when the wire vibrates in the presence of a transverse magnetic field. This emf is proportional to the integral of the wire's velocity over its length. Whitmore has shown that in the case of a wire driven by a short current pulse, only the fundathental mode of the wire's vibration contributes significantly to the observed emf. (29) In that case the contribution of the $m^{\text {th }}$ pair of normal modes to the observed emf is reduced by a factor of $1 / \mathrm{m}^{3}$ from the contribution of the fundanental. In our case similar considerations hold, and only the fundamental contributes significantly to the observed emf.

Assume that before the pulse the wire is in rotation but otherwise at rest. Then the boundary conditions for $\psi(z, t)$ are 


$$
\begin{array}{ll}
\Psi\left(z, t^{\prime}\right)=0, & 2-59 a \\
\frac{\partial \Psi\left(z, t^{\prime}\right)}{\partial t}=0, & 2-59 b \\
\Psi( \pm L / 2, t)=0 . & 2-59 c
\end{array}
$$

The approximate solution for the fundamental mode is found in the interval $t^{\prime} \leq t \leq 0$ to be

$$
\begin{aligned}
& \Psi_{1}(z, t)=\frac{+4 I_{0} B e^{i \pi / 4}}{\mu \pi\left[\left(\omega_{10}^{2}-\omega_{1}^{2}\right)^{2}+b_{1}^{2} \omega_{1}^{2}\right]^{1 / 2}} \cos \left(\frac{\pi z}{L^{*}}\right) \\
& \quad \times\left\{\frac{\cos B_{1}}{\cos \theta_{1}} e^{-b_{1}\left(t-t^{\prime}\right) / 2} \cos \left(\omega_{1}\left(t-t^{\prime}\right)+\theta_{1}\right)\right. \\
& \left.-\cos \left(\omega_{1}\left(t-t^{\prime}\right)+B_{1}\right)\right\}, \quad 2-60
\end{aligned}
$$

where

$$
\begin{array}{ll}
b_{1}=2 \lambda_{1}-1 \Delta \omega, & 2-61 a \\
\Delta \omega=\Delta \omega_{k}-\Delta \omega_{r}, & 2-61 b \\
\omega_{1}=\sqrt{\omega_{10}^{2}-\left(b_{1} / 2\right)^{2}}, & 2-61 c \\
B_{1}=\tan ^{-1}\left(\frac{\omega_{10}^{2}-\omega_{1}^{2}}{b_{1} \omega_{1}}\right), & 2-61 d
\end{array}
$$

and

$$
\theta_{1}=\tan ^{-1}\left(\tan \beta_{1}-b_{1} / 2 \omega_{1}\right) . \quad 2-61 e
$$


We note that for typical experimental parameters the phase angles $\beta_{1}$ and $\theta_{1}$ are given to good approximation by

$$
\begin{aligned}
& { }^{\theta_{1}}=0, \\
& B_{1}=0 .
\end{aligned}
$$

The initial conditions for the free decay of the excited wire are determined by the values of $\Psi_{1}(z, t)$ and $\partial \Psi_{1}(z, t) / \partial t$ at the instant the driver is shut off. Usual practice in this experiment was to drive the wire for four periods of the fundamental, so the initial conditions for free decay are found by evaluating $\psi_{1}(z, 0)$ and $a \Psi_{1}(z, 0) / \partial t$ since $t$ is zero at the instant the drive is shut off. Then, with $t^{\prime}=-4 T_{1}$, where $T_{1}=2 \pi / w_{1}$, we find

$$
\begin{aligned}
\Psi_{1}(z, 0)= & -\frac{4 I_{0} B A^{1 / 2}}{\mu \pi \omega_{1}\left(4 \lambda^{2}+(\Delta \omega)^{2}\right)^{1 / 2}} \\
& \times \cos \left(\frac{\pi z}{{ }^{*}}\right) e^{i \gamma_{1}},
\end{aligned}
$$

where

$$
\Lambda=1+e^{-8 \lambda T_{1}}-2 e^{-4 \lambda T_{1}} \cos \left(2 \Delta \omega T_{1}\right), \quad 2-63
$$

and 


$$
\begin{aligned}
y_{1}=\pi / 4 & +\tan ^{-1}\left(\frac{\Delta \omega}{2 \lambda}\right) \\
& -\tan ^{-1}\left(\frac{\tan \left(2 \Delta \omega T_{1}\right)}{e^{4 \lambda T_{1} \sec \left(2 \Delta \omega T_{1}\right)-1}}\right), \quad 2-64
\end{aligned}
$$

and

$$
\frac{\partial{ }_{1}(z, 0)}{\partial t}=0 \text {. }
$$

The phase of the fundamental is given by

Eq. 2-64. This quantity measures the angle through which the fundamental has rotated during the drive. In our experiment the maximum value of $\Delta \omega^{\text {is }}$ approximately $5 \mathrm{rad}-\mathrm{sec}^{-1}$ and the maximum value for $\lambda$ is approximately $3.5 \mathrm{sec}^{-1}$. For values of this order of magnitude it is found that the fundamental mode rotates, while being driven for a time $4 \mathrm{~T}_{1}$, approximately one-half of the angle through which it would have rotated in the same time had it been oscillating freely. As w111 be seen, this fact means that in principle we must make a correction for the time length of drive in computing the apparent circulation. This correction is very small, however, since $4 \mathrm{~T}_{1}$ is small compared to other times in the experiment.

The equation for the freely decaying wire for $t>0$ is found using Eqs. $2-62$ and 2-65. This equation is 


$$
\begin{aligned}
& \Psi_{1}(z, t)=\frac{4 I_{0} B^{1 / 2}}{\mu \pi \omega_{1}\left(4 \lambda^{2}+(\Delta \omega)^{2}\right)^{1 / 2}} \quad 44 \\
& \times \cos \left(\frac{\pi z}{L}\right) \cos \left(\omega_{1} t\right) e^{-\lambda t} e^{L\left(\gamma_{1}+\frac{(\Delta \omega)}{2} t\right)} .
\end{aligned}
$$

The emf generated by the wire as it oscillates in the transverse magnetic field is given by

$$
E(t) \approx-B \int_{-L^{*} / 2}^{L^{*} / 2} \operatorname{Re}\left(\dot{\psi}_{1} e^{-i \pi / 4}\right) \mathrm{dz} \quad 2-67
$$

We ignore terms proportional to the damping constant and the frequency splitting due to circulation and rotation since these terms are both small. We assume $\gamma_{1}=\pi / 4$. With these approximations the emf is

$$
\begin{gathered}
E(t)=-\left(\frac{4 B}{\pi}\right)^{2} \frac{2 I_{0} L^{*} \Lambda^{1 / 2}}{\mu\left(4 \lambda^{2}+(\Delta \omega)^{2}\right)^{1 / 2}} e^{-\lambda t} \sin \left(\omega_{1} t\right) \\
\times \cos \left(\frac{\Delta \omega}{2} t\right) .
\end{gathered}
$$

If $\Delta_{a}=0$ we observe on the oscilloscope screen a sinusoidal signal whose amplitude decays with decay constant $\lambda$. If $\Delta w_{K}-\Delta w_{r}$ is not zero the emf is a decaying sinusoidal beat signal with full amplitude modulation. The time between nodes 
is $2 \pi /\left(\Delta \omega_{k}-\Delta \omega_{r}\right)$. The emf observed is the decaying beat pattern generated by mixing two signals differing in frequency by $\Delta \omega_{K}-\Delta \omega_{r}$ with mean frequency $\omega_{1}$.

\section{Application to an Asymmetric W1re}

We now wish to discuss the motion of an asymetric wire while it oscillates freely after it has been excited. The wire is driven at the frequency $\omega_{10}$, corresponding to the lowest pair of normal modes. We assume that only the lowest pair of normal modes are significantly excited. We also assume that the Initial conditions for the free decay of the asymetric wire are the same as those found for the symmetric wire, i.e., at $t=0$, ignoring the effects due to the drive time,

$$
\Psi_{1}(z, 0)=c_{1} \quad e^{i \pi / 4},
$$

where $C_{1}$ is a real constant and

$$
\frac{\partial \psi_{1}(z, 0)}{\partial t}=0 \text {, }
$$

where

$$
\Psi_{1}(z, t)=\Psi_{1}^{+}(z, t)+\Psi_{1}^{-}(z, t), \quad 2-70
$$

and $\psi_{1}^{ \pm}$are given by Eq. 2-50. The inftial conditions then yield 


$$
\begin{aligned}
& A_{1}^{+}=\frac{C_{1}}{\sqrt{2\left(1+R_{1}^{2}\right)}}, \\
& A_{1}^{-}=\frac{R_{1} C_{1}}{\sqrt{2\left(1+R_{1}^{2}\right)}} \text {, } \\
& \operatorname{tan\phi _{1}^{+}}=\mathrm{R}_{1}=\mathrm{A}_{1}^{-} / \mathrm{A}_{1}^{+}, \\
& \tan \phi_{1}^{-}=-1 / R_{1}=-\Lambda_{1}^{+} / A_{1}^{-} .
\end{aligned}
$$

We note that under these conditions the modes are equally exeited.

The emf generated by the wire oscillating in the magnetic field is given by

$$
E=-B \frac{d}{d t} \int_{-L / 2}^{L / 2} \operatorname{Re}\left(\psi_{1}(z, t) e^{-1 \pi / 4}\right) d z,
$$

where $\operatorname{Re}\left(\psi_{1}(z, t) e^{-i \pi / 4}\right)$ is the projection of $\psi_{1}(z, t)$ in the direction perpendicular to the magnetic field. Substituting from Eqs. $2-50$ and $2-71$ we obtain

$$
\begin{aligned}
\operatorname{Re}\left(\psi_{1}(z, t) e^{-i \pi / 4}\right) & =\frac{f_{10}(z, t) e^{-\lambda t} C_{1}}{2} \\
& \times\left[\cos \left(\omega_{10}^{+} t\right)+\cos \left(\omega_{10}^{-} t\right)\right],
\end{aligned}
$$

where we have assumed $\lambda^{+}=\lambda^{-}=\lambda$. The emf is then

$$
E=B C_{1} \int_{-L / 2}^{L / 2} f_{10}(z) d z e^{-\lambda t} \omega_{10} \sin (\omega, 10 t) \cos \left(\frac{\Delta \omega}{2} t t\right) \cdot 2-72
$$

The emf generated is a decaying beat pattern with carrier frequency w $10^{\circ}$ The time between nodes in the pattern is $2 \pi / \Delta \omega_{1}$. 


\section{The Direction of Circulation}

The direction of circulation is found by impressing upon the magnetic fleld $\vec{B}$ a small perturbing field $\vec{B}^{\prime}$ at $90^{\circ}$ with respect to the direction of $\vec{B}$ and approximately $10 \%$ of the magnitude of $\vec{B}$. This perturbing field is imposed during the excltation only. During the free decay only the field $\vec{B}$ is present.

Consider the case of the symmetric wire in the model calculation. If, for example, under these conditions, the direction of the resultant field $\vec{B}+\vec{B}$ ' is $\pi / 4+\delta$, then the angle $\gamma_{1}$ in Eq. $2-64$ is increased by $\delta$.

$$
\text { If } \Delta w_{k}-\Delta \omega_{r}>0 \text {, the first node in the }
$$
pattern occurs at time $\tau_{n}$ such that

$$
\delta+\frac{1}{2}\left(\Delta \omega_{K}-\Delta \omega_{r}\right) \tau_{n}=\pi / 2,
$$

and the time from the excitation of the wire to the first node is reduced. However, the time between successive nodes is not changed. Similarly, if $\Delta \omega_{K}-\Delta w_{r}<0$, the time to the first node is longer than the usual case. Thus, by pulsing the field so as to move the excftation plane in a known direction and observing whether the time to the 
first node is decreased or increased from its steady value determines the sign of the quantity $\Delta \omega_{K}-\Delta \omega_{r}$. In the case of the symmetric wire the amplitude is fully modulated and the displaced node is a true amplitude nu11.

In the case of the asymmetric wire, the beats between $\psi^{+}$and $\psi^{-}$arise because of the shifting phase difference between $\psi^{+}$and $\psi^{-}$. The phase of $\psi^{+}$changes relative to that of $\psi^{-}$at a rate $\Delta \omega_{1}$. Further $\varphi^{+}$always moves in the direction given by the sign of the quantity $\Delta \omega_{K}-\Delta \omega_{r}$, i.e., counterclockwise if $\Delta \omega_{K}-\Delta \omega_{r}>0$, and clockwise if $\Delta w_{K}-\Delta w_{r}<0$. If we assume that $\Delta w_{K}-\Delta w_{r}>0$ and that the plane of excitation is advanced in the counter clockwise direction from the usual plane of excitation by the perturbing field, then the phase of the mode $\psi^{+}$is advanced and that of $\psi^{-}$is retarded. The phase of the resultant is advanced and the observed time to the first node is decreased. Similarly, if the plane of excitation is retarded, the phase of $\psi^{+}$is retarded and that of $\psi^{-}$is advanced; the time to the first mode is increased. Thus, by knowing the direction in which the plane of excitation is rotated and observingthe change in time to the node, the sign of the quantity $\Delta w_{k}-\Delta w_{r}$ 
can be determined.

It should be noted that when field $B_{1}$ is used in the case of the asymmetric wire the two modes are not equally excited and consequently the node observed in the beat pattern is not a true nu11. However, the direction in which the node is moved can still be observed.

\section{Experimental Considerations}

Since, as we have seen, only the lowest modes of the wire are observed, we now simplify the notation of Eq. 2-34. The frequency splitting between the two modes $\Delta \omega_{m t}$ will now be written $\Delta \omega_{t}$, where the subscript $t$ implies the total frequency splitting. The frequency splitting $\Delta \omega_{\text {mo }}$ will now be given as $\Delta \omega_{0}$, and referred to as the intrinsic frequency splitting. The frequency splitting due to circulation $\Delta \omega_{\text {mK }}$ will now be given as $\Delta w_{k}$. With this change in notation Eq. $2-42$ is written as

$$
\Delta \omega_{t}=\sqrt{\left(\Delta \omega_{0}\right)^{2}+\left(\Delta \omega_{K}-\Delta \omega_{r}\right)^{2}} \cdot \quad 2-73
$$

The beat frequency of the wire $\Delta w_{t}$ could, in principle, be determined by measuring the time between successive nodes in the beat pattern. However, the damping introduced by the normal fluid 
complicates the measurement. As can be seen from Tables Ia to Ic, a typical damping time for the wire was $300 \mathrm{msec}$. Under our running conditions the wire signal would decay into the noise in about three damping times. Thus, in order to observe two nodes in the beat pattern, $\Delta \omega_{0}$ would have to be such that $3 \pi / \Delta \omega_{0} \approx 1 \mathrm{sec}$ or $\Delta \omega_{0} \approx 3 \pi \mathrm{rad} / \mathrm{sec}$. Since the quantity $\Delta w_{K}-\Delta w_{r}$ ranged from $0 \mathrm{rad} / \mathrm{sec}$ to $4 \mathrm{rad} / \mathrm{sec}$ in our measurements, the maximum change which circulation and rotation would bring about under these conditions would only be $10 \%$ and under most conditions would be considerably smaller. Because of this we chose to measure the time from wire excitation to the first node in the beat pattern. Then $\Delta \omega_{0}$ could be as small as $\pi \mathrm{rad} / \mathrm{sec}$, and considerably larger changes in $\Delta w_{t}$ would be observed for the same changes in $\Delta \omega_{k}-\Delta \omega_{r}$. As Is discussed in Appendix II, changes in $\Delta \omega_{0}$ were brought about by twisting the wire in a set-up run previous to runs in which the circulation was measured, and by varying the tension of the wire during normal runs. 


\section{CHAPTER III}

\section{PROCEDURE}

\section{Description}

The experimental procedure followed during a typical run varied very little from run to run.

A schematic diagram of the lower part of the experimental apparatus is shown in Fig. 3. The apparatus was cooled overnight to liquid nitrogen temperature. The helium dewar was flushed three times with helium gas in order to assure that the magnet would not freeze to its support and would be free to rotate. Before beginning to transfer helium the nitrogen gas put into the vacuum can to facilitate heat exchange would be pumped out, and the vacuum can would be leak-checked. A few $\mathrm{cm}^{3}$ of hydrogen gas were then put into the vacuum can to act as an exchange gas. The transfer of liquid helium was begun. The pressure of the hydrogen gas in the vacuum can was monitored with a thermocouple gauge. When this pressure began to drop, indicating that the hydrogen gas was condensing, the transfer rate was reduced, and the hydrogen pressure would rise. This suggested that parts of the apparatus within the vacuum can were warmer than the conden- 


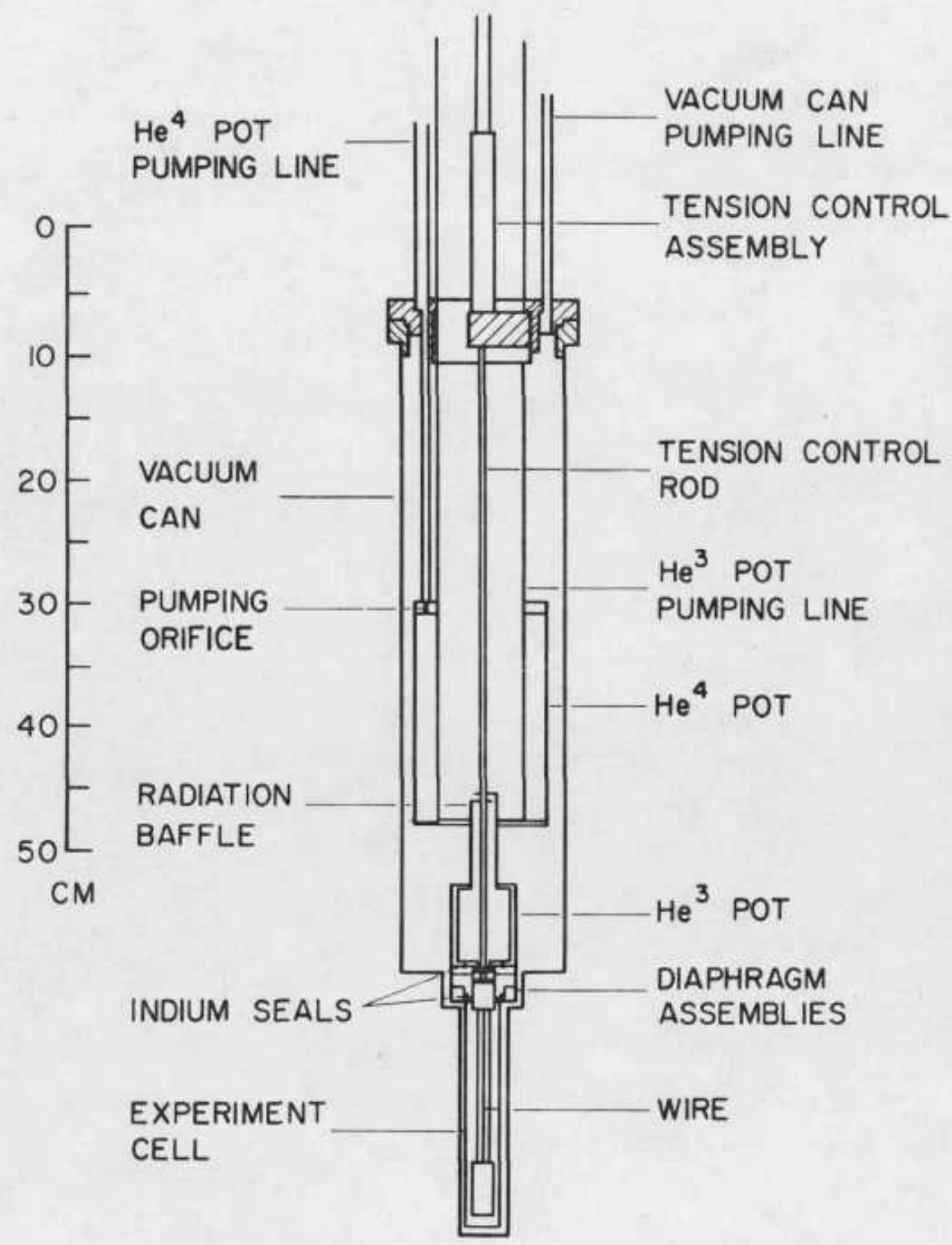

PIGURE 3 - Schematic of Lower Part of Apparatus 
sation temperature of the hydrogen. After a few cycles of decreasing and increasing the transfer rate, the hydrogen gas would remain condensed and the transfer would proceed. Almost immediately liquid helium would begin to collect.

When the liquid level was disceraible at the bottom of the dewar the He ${ }^{4}$ pot fill valve would be shut and the gas in the pot would be evacuated. When the liquid level had risen high enough to cover the $\mathrm{He}^{4}$ pot fill valve, this valve would be opened and liquid would enter the pot. The pot would then be pumped throughout the filling process, and the temperature of the pot during filling, as determined from the resistance of the thermometer, was kept below the lambda temperature. The pressure in the pumping line at room temperature was monitored with a 0-50 torr gauge and kept at 4 torr pressure. After some 15 or 20 minutes the pot would be filled. Two different indicators could be used to show that the pot was filled. The He ${ }^{4}$ pot temperature would be very stable during the filling process at about $1.8 \mathrm{~K}$. After about 15 minutes the temperature would suddenly begin to rise. At the same time the pressure, as read on the 0-50 torr gauge, would show rapid fluctuations a few torr in size and then begin 
to rise rapidly. Both of these effects were thought to be due to a column of liquid rising in the $\mathrm{He}^{4}$ pot pumping line above the orifice at the output of the $\mathrm{He}^{4}$ pot. As the 1 iquid level rose, it would come in contact with sections of the pumping line at higher temperature. It would begin to boil away more rapidly. It would also act as a thermal conductor, conducting heat down to the liquid in the $\mathrm{He}^{4}$ pot, causing the temperature to rise. Once the $\mathrm{He}^{4}$ pot was filled the fill valve would be closed and its temperature would begin to drop.

The thermometer on the $\mathrm{He}^{3}$ pot and the thermometer on the sample chamber indicated, at this time, that they were still warm, near the temperature at which the hydrogen gas had condensed. The tension control assembly was also warm since it had no means of cooling, being located on the axis of the He ${ }^{3}$ pot pumping line which had been kept evacuated during the entire cool-down period.

$$
\mathrm{He}^{3} \text { gas from its storage can was introduced }
$$
into the $\mathrm{He}^{3}$ pumping line. Care had to be taken at this point to do this slowly, since the cooling of the warm $\mathrm{He}^{3}$ pot, sample chamber, and tension control assembly boiled away a significant amount of liquid 
helium from the $4 \mathrm{~K}$ bath. Enough He${ }^{3} \mathrm{gas}$ was introduced to insure that 11 quid would form in the $\mathrm{He}^{3}$ pot.

At this point the persistent current magnet was energized. Two power supplies were used, one to provide the 2 A current to the magnet and one to supply the $15 \mathrm{~mA}$ current to the persistent current switch. The wire was driven with 1 msec, $5 \mathrm{~V}$ pulses, and the emf it generated was used as an indicator that the magnetic field had been estab1ished. The current to the persistent current switch was then shut off, and after a $15 \mathrm{sec}$ wait the current to the magnet was shut off. This wait was necessary to allow the switch to cool and the shunt to become superconducting.

The final step in the initlal stages of the run was the filling of the sample chamber. In all the runs described here the sample chamber was filled from a ballast can at room temperature containing vapor under high pressure. The volume of the sample chamber and of the fill capillaries, up to the capillary wound about the He ${ }^{4}$ pot was computed. Then the number of moles of $\mathrm{He}^{4}$ needed to fill this volume when condensed was computed. Using these results, it was found that if the 
ballast can were filled with helium gas at 230 $1 b / i n^{2}$ gauge pressure, then when this gas was condensed, it would just fill the available volume. The ballast can was filled to this pressure, and the gas was allowed to condense into the cell. In about 20 minutes the system would be filled. During the filling procedure the wire's amplitude of oscillation for a fixed drive was monitored. The increase in the damping which occurred when the cell was filling was used as a sign that the fill capillary was not blocked. In order to make sure the sample can was filled, some fillings were made from the ballast can when the gas was at higher pressures. When the pressure in the ballast can at room temperature was increased to $2501 \mathrm{~b} / \mathrm{in}^{2}$ gauge pressure the pressure of the vapor above the liquid which formed at condensation would rise to 1 atm, indicating that the liquid level had risen above the $\mathrm{He}^{4}$ pot to the $4 \mathrm{~K}$ bath. Once the sample chamber was filled, the system was allowed to cool to its equilibrium temperature. After equilibrium was established, measurement of the wire's properties could begin. The first step was to choose a running frequency. In accord with Whitmore's results no frequencies less than 
$500 \mathrm{~Hz}$ were used, since he found that these did not show plane-polarized modes in the absence of circulation.

The primary consideration in choosing a running frequency greater than $500 \mathrm{~Hz}$ was the value of the intrinsic frequency splitting Aus at that running frequency. To determine this splitting the node times as a function of frequency were measured at this time. Once these node times were obtained a running frequency could be obtained.

Two criteria were used in selecting this running frequency. It is desirable to choose a running frequency such that the intrinsic frequency splitting $\Delta w_{0}$ will be as small as possible yet still be observable. In practice, when the running frequency was chosen it was not known whether circulation was present or not. Because of this fact, we would choose that frequency at which the measured frequency splitting $\Delta \omega_{t}$ was smallest, yet still observable. Then the rate of change of the measured frequency splitting with frequency around the chosen frequency was determined. It was necessary that this be small since there was some drift in wire frequency during the run and it was desirable that small changes in wire frequency have a negligible effect on $\Delta \omega_{0}$. 
General1y, once a running frequency was chosen this frequency was used throughout the run, which often lasted a few weeks.

However, on some occasions there were abrupt changes in the behavior of $\Delta \omega_{t}$ as a function of frequency. During a long run with wire $B$ an accidental warm-up of the sample cell occurred. After the system was cooled again it was found that the curve of $\Delta \omega_{t} v s$. frequency had changed, and that for a given frequency the value of $\Delta \omega_{t}$ was larger than it had been. The same effect was seen in an earlier run with wire A when solid helium was formed in the cell. The high pressure required to form the solid also caused the fiber to be under high tension, due to flexing of the diaphragm at the top of the experimental cell. When the solid was melted and running conditions reestablished it was found that at the running frequency $\Delta \omega_{t}$ had increased. During the last run with wire C efforts were made to bring about changes in $\Delta w_{t}$ by rapping the system sharply with a hamer. When this was done, it was found that the slope of the curve of $\Delta \omega_{t}$ versus frequency became very large. Indeed, the rate of change of $\Delta \omega_{t}$ with frequency became so large that 
the run had to be terminated. The origin of this experimental difficulty is not known, but any sticking of the upper post or tension control assembly could expluin it.

After a running frequency had been established, the tuned amplifier would be tuned to that frequency by using the Lissajous figure obtained when the input and output signal of the amplifier were impressed on the horizontal and vertical deflection plates of an oscilloscope. For the amplifier used a trace corresponding to a phase shift of $0^{\circ}$ was observed upon proper tuning. Checks made during the run showed that the amplifier was quite stable with respect to tuning. Once the amplifier was tuned, its free decay time was obtained by measuring the halving time of its ringing response to a voltage impulse. The decay time of the wire was obtained by measuring the halving time in the decay of its response to a voltage impulse.

\section{The Wire Drive Signal.}

The drive signal used to excite the wire was chosen on the basis of a number of considerations. First, the displacement amplitude of the wire oscillation produced had to be large enough to allow observation of the node before the signal decayed 
into the noise. However, the drive producing the displacement could not be so large as to disturb the circulation around the wire or to produce undesirable heating. In determining the power level used in the wire drive signal, the observation made by Whitmore in his work that when the power level exceeded $1 \mathrm{~mW}$, the stability of the circulation appeared to be disturbed, was used. In accord with this finding, all power levels to the wire were kept sma11, the largest being $150 \mu \mathrm{W}$. The actual power delivered to the wire in the runs discussed is 1 isted in Tables Ia - Ic.

The maximum displacement amplitude and velocity amplitudes produced by the drive signal can be obtained from Eq. 2-62.

The displacement amplitude and velocity amplitude of the wire are simply related. If $r_{0}$ is the displacement amplitude, then $v_{0}=w r_{0}$ is the velocity amplitude. In attempting to establish a criterion in choosing a velocity amplitude it has been suggested that the maximum velocity of the wire be less than the superfluid velocity at the wire's surface for one quantum of circulation. This criterion requires wro $\leq h / 2 \pi a m$, or, for the wires used here, $\omega \mathrm{r}_{0} \leq .03 \mathrm{~cm} / \mathrm{sec}$. 
In the actual experiment the velocity amplitude was usual1y near to twice this value, in accordance with the results of Whitmore's work in which it was found that velocity amplitudes of this size did not effect the circulation.

Studies of the effect of large velocity amplitudes on the measured circulation were made with wire B. In these studies $1 \mathrm{t}$ was found that repeated pulses producing velocity amplitudes as large as $1 \mathrm{~cm} / \mathrm{sec}$ would usually produce no change in the measured circulation. However, for larger pulses, producing velocity amp1itudes as large as $10 \mathrm{~cm} / \mathrm{sec}$, it was found the repeated pulses would induce changes in the circulation level, although single pulses of this size would not always produce circulation changes.

As a last step in the process of adjusting the wire drive signal, the interval between successive excitations of the wire was adjusted to be five or six seconds. The five-second interval was used when node times were obtained from visual observations, and the six-second interval, which corresponds to 10 observations per minute, was used when the automatic datataking system was used.

At this point in the procedure the automatic datataking system was adjusted (see Appendix I) and measurements of the time to the first node were begun. 
If the system was to be rotated, the rotational drive was connected. Then, at some time after the beginning of the recording of node times, the rotation would begin. At selected intervals, never exceeding five minutes, the time for one rotation would be recorded. Periodically the direction finder would be pulsed on, and the change in time to the first node would be recorded.

As a means of correlating all this information, a timer reading to 0.1 minute was used. The timer would be started when data-taking began and was the primary means of relating all measurements.

\section{Determination of the Intrinsic Frequency}

\section{Splitting}

After the running frequency was chosen the value of $\Delta \omega_{t}$ found at that frequency would vary slightly from day to day, and no satisfactory way was found to assign a value to the intrinsic frequency splitting $\Delta \omega_{0}$ from measurements made at rest. After a number of runs in rotation a method of assigning a value to $\Delta \omega_{0}$ was found. In the course of many of the runs, the sense of the rotation would be changed so that the system would be rotated in both directions. It was observed that if the system was rotated first in one direction, then brought to rest, 
and then counter-rotated, with either a small acceleration or at a fixed rotation speed greater than $0.2 \mathrm{rad} / \mathrm{sec}$, the time to the first node, which is equal to $\pi / \Delta w_{t}$, would always go through a characteristic and reproducible maximum. This maximum would generally occur at a rotation speed in the range from 0.17 to $0.23 \mathrm{rad} / \mathrm{sec}$ in absolute value. The origin of the maximum can be understood from the expression for the frequency splitting,

$$
\Delta \omega_{t}=\sqrt{\left(\Delta \omega_{0}\right)^{2}+\left(\Delta \omega_{K}-\Delta \omega_{r}\right)^{2}} \text {. }
$$

When the circulation and the rotation have the same sense, and the frequency splittings due to them have the same magnitude, their contributionsto the total frequency splitting cancel, yielding a minimum in $\Delta w_{t}$ and consequently a maximum in the time to the node. This method was used to determine $\Delta w_{0}$ in a11 runs when the opportunity to observe this behavior occurred. In some runs the rotation speed would pass through zero twice and the passage of the node time through a maximum value would be repeated. The node times as the system passed through these maxima were averaged over one minute, as were al1 the data. When this was done, these averaged node times were within elght milliseconds of each other, in every case. 
4. Data Reduction and Analysis

After a run, the data obtained consisted of a series of times to the first node in the beat pattern, rotation speeds, and observations of the change in the time to the first node when the direction finder was used; all these data were correlated with the time into the run as reac on the timer.

There were two kinds of runs made in the course of the experiment. In the early stages of the experiment, data would be taken at fixed rotation speeds. The time to the first node would be recorded from visual observations of the wire's emf as displayed on the screen of an oscilloscope. The wire was excited every five seconds. At a given rotation speed, data would be taken over a sufficiently long time to assure that no changes of the node time were taking place. The rotation speeds were obtained automatically from a time-interval counter which was triggered on and off by a signal originating from a photo-sensitive diode. This signal was generated by the passage of a beam of light over the diode. This beam originated from a stationary source and was reflected off a mirror on board the rotating turntable onto the stationary diode. 
The time for the single rotation was printed by a line printer.

In the later runs the system was accelerated continuously. With the exception of the last run using wire $B$ the time to the first node was acquired using the automatic system and recorded by the line printer. When the automatic system was used the wire was excited every six seconds; the rotation speed in these runs was recorded by hand.

In the first type of run, data analysis was straightforward. Using the data of Reynolds, et. a1., the value of $p_{s}$ at the running temperature was determined. $(30)$ The value of $\mu$, the effective mass per unit length of the wire, was determined as described in Appendix II. The measured time to the node was taken from the visually recorded data. The necessary corrections because of amplifier delay (see Appendix I) and drive time were made. The value of the frequency-splitting due to rotation was calculated using the measured time per rotation and Eq. 2-41d. The intrinsic frequency splitting was determined as discussed earlier, or if this was not possible the value last obtained using that method was used. Then the value of the circulation in units of $\mathrm{h} / \mathrm{m}$ was calculated 
using Eq. 2-73. It should be noted that this equation is quadratic in the frequency splitting due to circulation, and consequently two values of the apparent circulation were found for each value of the total frequency splitting. The results of the measurement of the sense of the quantity $\Delta \omega_{K}-\Delta \omega_{r}$ were used to determine which value was appropriate. The result of a single sense determination was somewhat ambiguous. Consider the case when the perturbing field caused the plane of excitation to be rotated counter-clockwise. Then if the system were rotating counter-clockwise and the direction finder showed that the time to the first node was reduced, this would indicate that the quantity $\Delta \omega_{K}-\Delta \omega_{r}$ was positive. The only possible interpretation in this case is that $\Delta \omega_{K}$ was positive, 1.e., that the sense of the circulation was the same as that of the rotation. However, it was possible that the time to the first node was increased. There would then be two possible interpretations. The first is that $\Delta \omega_{K}$ was positive but less than $\Delta \omega_{r}$. The second is that $\Delta \omega_{k}$ was negative. Since the exact amount of the change in the time could not be easily found, this ambiguity could not be resolved from a single direction measurement. However, we can make 
use of the observation made in our work that the circulation changes went smoothly. Consequently we assumed that as we moved from a region where the interpretation of the direction finder was unambiguous into a region where a possible ambiguity existed, any circulation changes took place smoothly. In addition, the sense of the circulation was thought to have changed only after the total frequency splitting went through the characteristic minimum discussed before. In this way the apparent circulation could be determined in both magnitude and direction.

In the second type of run the time to the first node was recorded every six seconds by the line printer while the system was undergoing a continuous acceleration. The time for each rotation of the apparatus was recorded by hand. These times were then fitted to a curve of the form $t_{1}=A t+B+C / t$, where $t_{1}$ is the time for a single rotation and $t$ is the clock time into the run as recorded by the timer. The node times were punched on cards to facilitate computer analysis. Using the computer, these times were taken in lots of ten and averaged, ylelding the average time to the node for each minute of the run. Since the data were taken automatically and 
there was some noise present, the system would sometimes record a node time which was spurious. To eliminate these points from the averaging process, only those node times falling within an acceptable range were included in the averaging. This range was established by visual observation of the maximum and minimum node times in the run. Only in those minutes in which six or more node tines fell within this range were the data averaged and used in calculating the circulation.

Then, using the computer, the apparent circulation was calculated from Eq. 2-73. The center of the minute over which the node times were averaged was matched with the rotation speed at that time as calculated from the function to which the rotation speeds had been fitted. The correction for delay was made.

An added advantage of the computer analysis was the use of the computer's line printer to plot the apparent circulation as a function of both time and rotation speed. In this way we were often able to have the complete results of a run within a few days of the run. 


\section{CHAPTER IV}

\section{RESULTS}

\section{Results}

Data were obtained using three wires. The properties of these wires are listed in Tables Ia through Ic. Also included in the tables are the values of a number of parameters which are measured during a run. Each run is identified with a letter (A, B, or C) which identifies the wire used in that run and a number. The numbers are assigned chrono$\log i \mathrm{cal} 1 \mathrm{y}$.

The term "run" is used here to describe a period in which data were taken. In some cases, many runs were made with the same sample. For example, runs B-1 through B-14 were all made using the same sample of liquid helium.

As mentioned in the previous chapter, two types of runs were made. In the first type the rotation speed was changed in a step-like manner, and the circulation was measured at a series of fixed rotation speeds. In the second type of run the system underwent smooth acceleration and deceleration, and the circulation was measured over the entire range of rotation speeds traversed. 
$\underline{\text { TABLE Ia }}$

\section{EXPERIMENTAL PARAMETERS FOR RUNS WITH WIRE A}

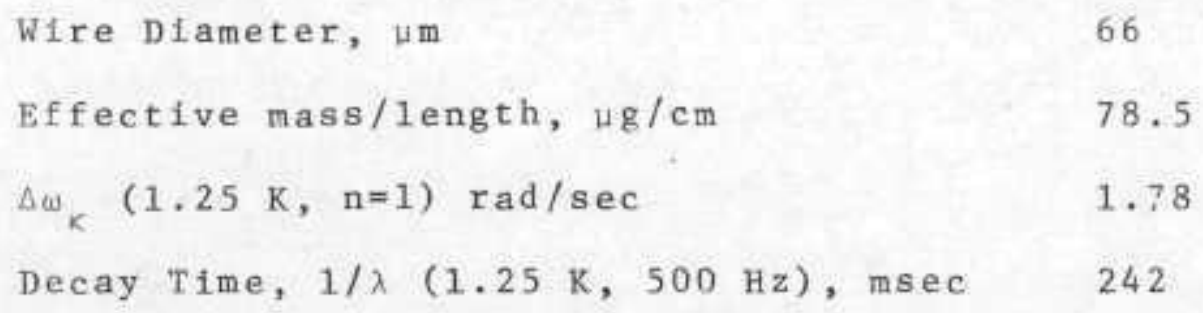

\begin{tabular}{lcc}
\hline Run Number & 1 & 2 \\
\hline Wire frequency, $\omega / 2 \pi \mathrm{Hz}$ & 592 & 592 \\
$\Delta \omega_{0}, \mathrm{rad} / \mathrm{sec}$ & 7.11 & 6.57 \\
Time delay, msec & 23.5 & 7.52 \\
Power in drive, $\psi W$ & 80 & 82 \\
Run Temperature, $\mathrm{K}$ & 1.35 & 1.33
\end{tabular}


TABLE Ib

EXPERIMENTAL PARAMETERS EOR RUNS WITH WIRE B

$\begin{array}{ll}\text { Wire Diameter, } \omega \mathrm{th} & 78.8 \\ \text { Effective mass/length, } \mu \mathrm{g} / \mathrm{cm} & 136.9 \\ \Delta \omega_{K}(1.25 \mathrm{~K}, \mathrm{n}=1) \mathrm{rad} / \mathrm{sec} & 1.015\end{array}$

Decay Time, $1 / \lambda(1.25 \mathrm{~K}, 500 \mathrm{~Hz})$, msec 365

\begin{tabular}{|c|c|c|c|c|c|c|c|c|c|c|}
\hline Run Number & 1 & 2 & 3 & 4 & 5 & 6 & 7 & 8 & 9 & 10 \\
\hline Wire frequency, $\omega / 2 \pi \mathrm{Hz}$ & 572 & 572 & 572 & 572 & 572 & 572 & 572 & 572 & 572 & 572 \\
\hline$\Delta \mathrm{dit}_{0}, \mathrm{rad} / \mathrm{sec}$ & 3.73 & 3.77 & 3.79 & 3.67 & 3.72 & 4.49 & 4.27 & 4.28 & 4.28 & 4.27 \\
\hline Time delay, msec & 27.5 & 38.5 & 32.0 & 37.1 & 7.68 & 42.5 & 7.40 & 10.1 & 9.55 & 6.62 \\
\hline Power in drive, $\mu W$ & 4.0 & 1.0 .4 & 12.2 & 41.6 & 18.4 & 28.8 & 28.2 & 26.1 & 26.1 & 26.1 \\
\hline Run Temperature, $\mathrm{K}$ & 1.23 & 1.23 & 1.24 & 1.23 & 1.23 & 1.23 & 1.23 & 1.23 & 1.23 & 1.23 \\
\hline
\end{tabular}




\section{TABLE Ib (Continued)}

EXPERIMENTAL PARAMETERS FOR RUNS WITH WIRE B

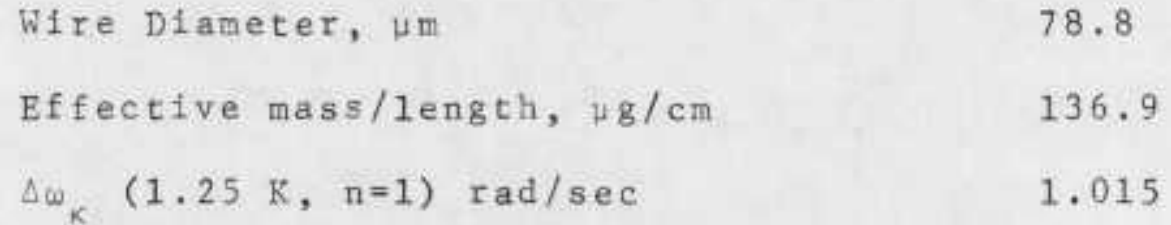

\begin{tabular}{|c|c|c|c|c|c|c|c|}
\hline Run Number & 11 & 12 & 13 & 14 & 15 & 16 & 17 \\
\hline Wire frequency, $\omega / 2 \pi \mathrm{Hz}$ & 572 & 572 & 572 & 572 & 526 & 526 & 525 \\
\hline$\Delta \omega_{0}, \mathrm{rad} / \mathrm{sec}$ & 4.27 & 4.32 & 4.20 & 4.20 & 4.10 & 4.10 & 4.05 \\
\hline Time delay, msec & 5.59 & 5.73 & 5.90 & 5.90 & 8.98 & 7.66 & 5.97 \\
\hline Power in drive, $\mu W$ & 26.1 & 26.1 & 45.1 & 45.1 & 65 & 88.5 & 88.5 \\
\hline Run Temperature, $\mathrm{K}$ & 1.23 & 1.23 & 1.23 & 1.23 & 1.32 & 1.31 & 1. 24 \\
\hline
\end{tabular}


TABLE IC

\section{EXPERIMENTAL PARAMETERS FOR RUNS WITH WIRE C}
Wire Diameter, $\mu \mathrm{m}$
83.3
Effective mass/length, $\mu \mathrm{g} / \mathrm{cm}$
136.3
$\Delta \omega_{k}(1.25 \mathrm{~K}, \mathrm{n}=1) \mathrm{rad} / \mathrm{sec}$
1.02
Decay Time, $1 / \lambda(1.25 \mathrm{~K}, 500 \mathrm{~Hz})$, msec 348

\begin{tabular}{lccccc}
\hline $\begin{array}{l}\text { Run Number } \\
\text { Wire frequency, } \omega / 2 \pi \mathrm{H}_{z}\end{array}$ & 504 & 504 & 504 & 504 & 504 \\
\hline $\begin{array}{l}\Delta \omega_{0}, \mathrm{rad} / \mathrm{sec} \\
\text { Time delay, msec }\end{array}$ & 10.62 & 12.64 & 10.6 & 10.4 & 11.98 \\
Power in drive, $\mu W$ & 115 & 146 & 115 & 65 & 65 \\
Run Temperature, K & 1.27 & 1.27 & 1.27 & 1.27 & 1.27
\end{tabular}


In the early runs circulation measurements were made at rotation speeds ranging from $3.5 \mathrm{rad} / \mathrm{sec}$ to $-3.5 \mathrm{rad} / \mathrm{sec}$. Wires $A$ and $B$ were both used in runs of this type. The results of these runs are shown in Fig. 4 and Fig. 5, Also shown in Fig. 5 are the results of the first run in which a smooth acceleration was used.

Fig. 6 through Fig. 10 show some of the data obtained with wire C. These data were obtained under conditions in which the system underwent constant accelerations. An 111ustration of how the data are analyzed is contained in F1gs. 6, 7, and 8. In Fig. 6 the time to the first node, averaged over one-minute intervals, is plotted against time into the run. This plot is used to determine $\Delta \omega_{0}$ for the run. At 337 minutes and at 572 minutes peaks in the data are seen, in each case soon after the rotation speed passed through zero. In the run shown the peaks both have maximum values of 842 msec, and this was the value taken to correspond to the intrinsic frequency splitting. Using the intrinsic frequency splitting found from thesedata the apparent circulation in units of the circulation quantum $\mathrm{h} / \mathrm{m}$ is plotted against time into the run in Fig. 7. In Fig. 8 these apparent circulation 


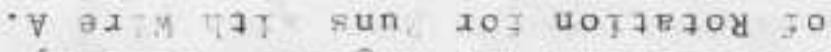

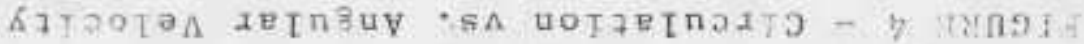

\section{APPARENT CIRCULATION IN UNITS OF $\mathrm{h} / \mathrm{m}$}

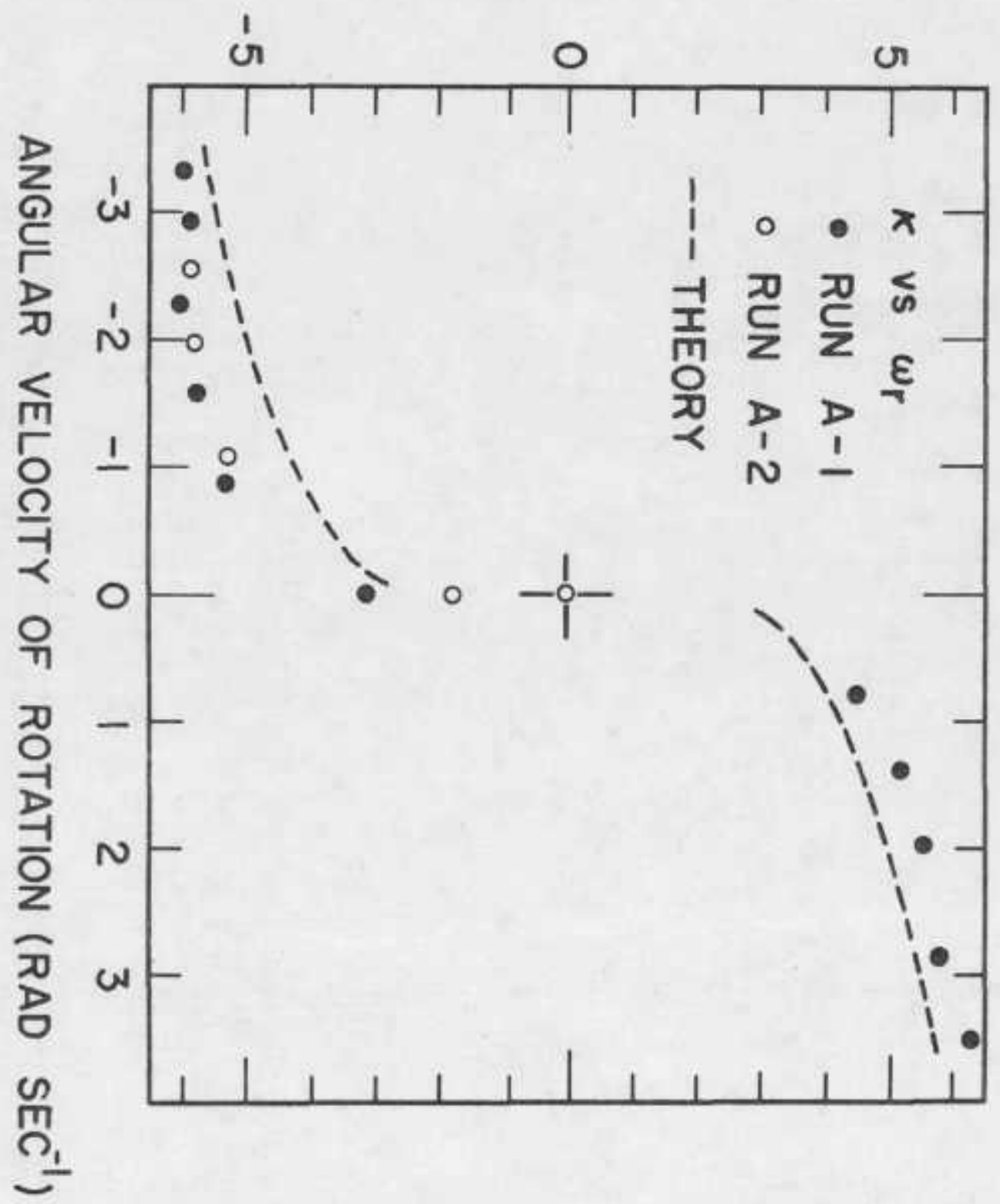




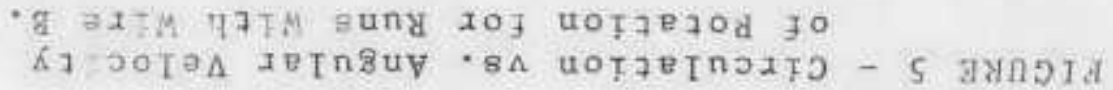

APPARENT CIRCULATION IN UNITS OF $\mathrm{h} / \mathrm{m}$

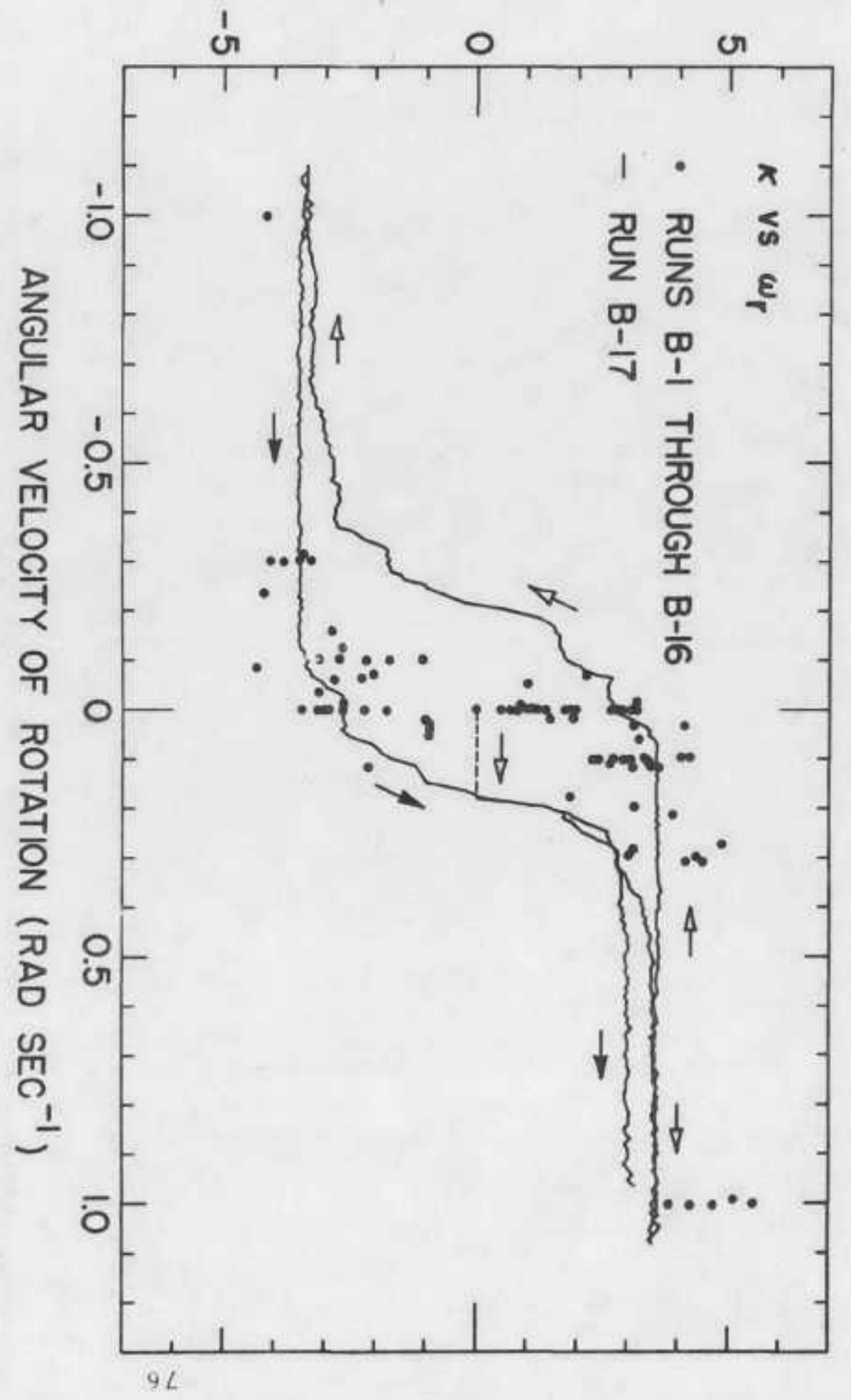




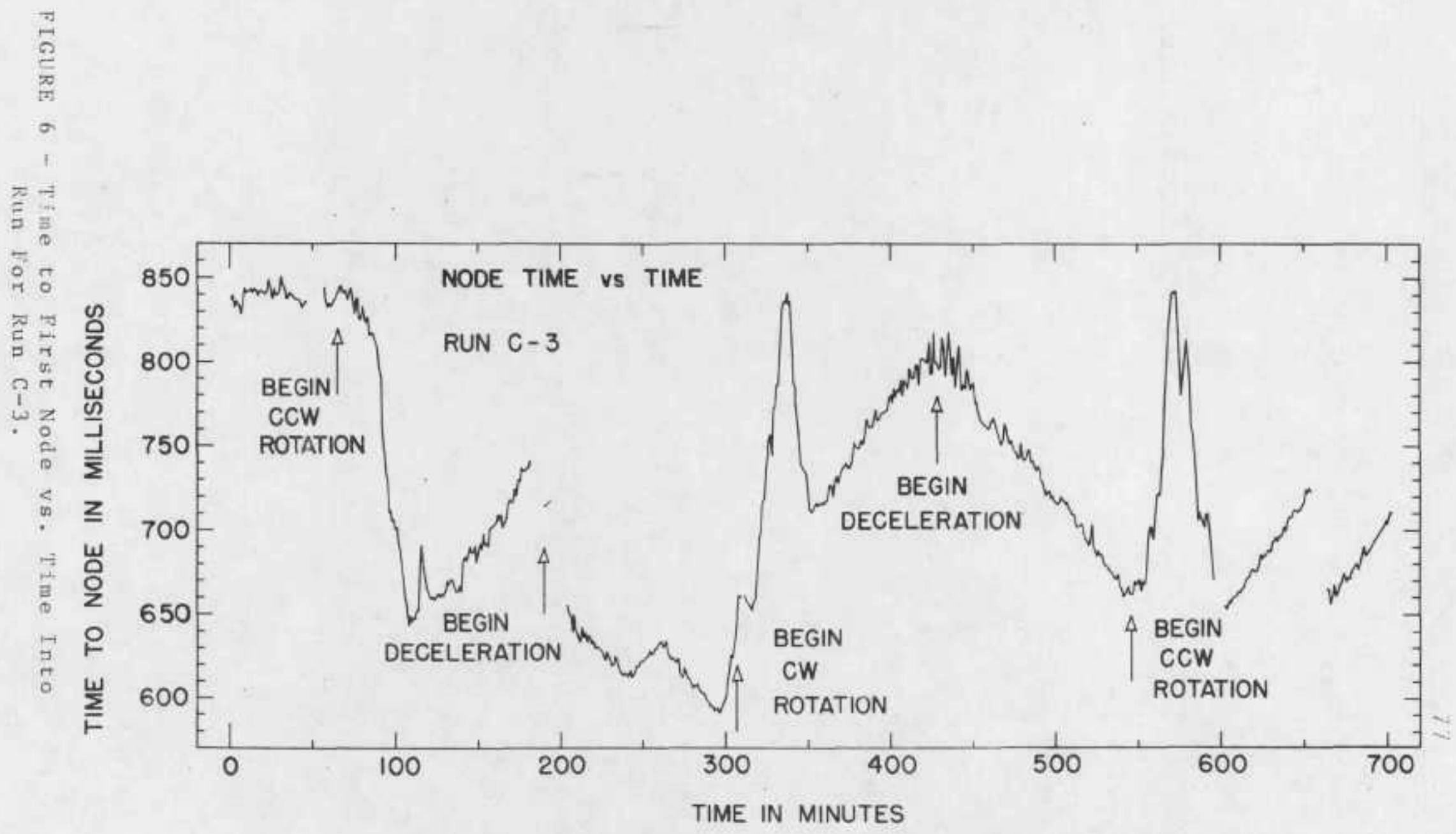


S $\$ กNIW NI $\exists W I \perp$

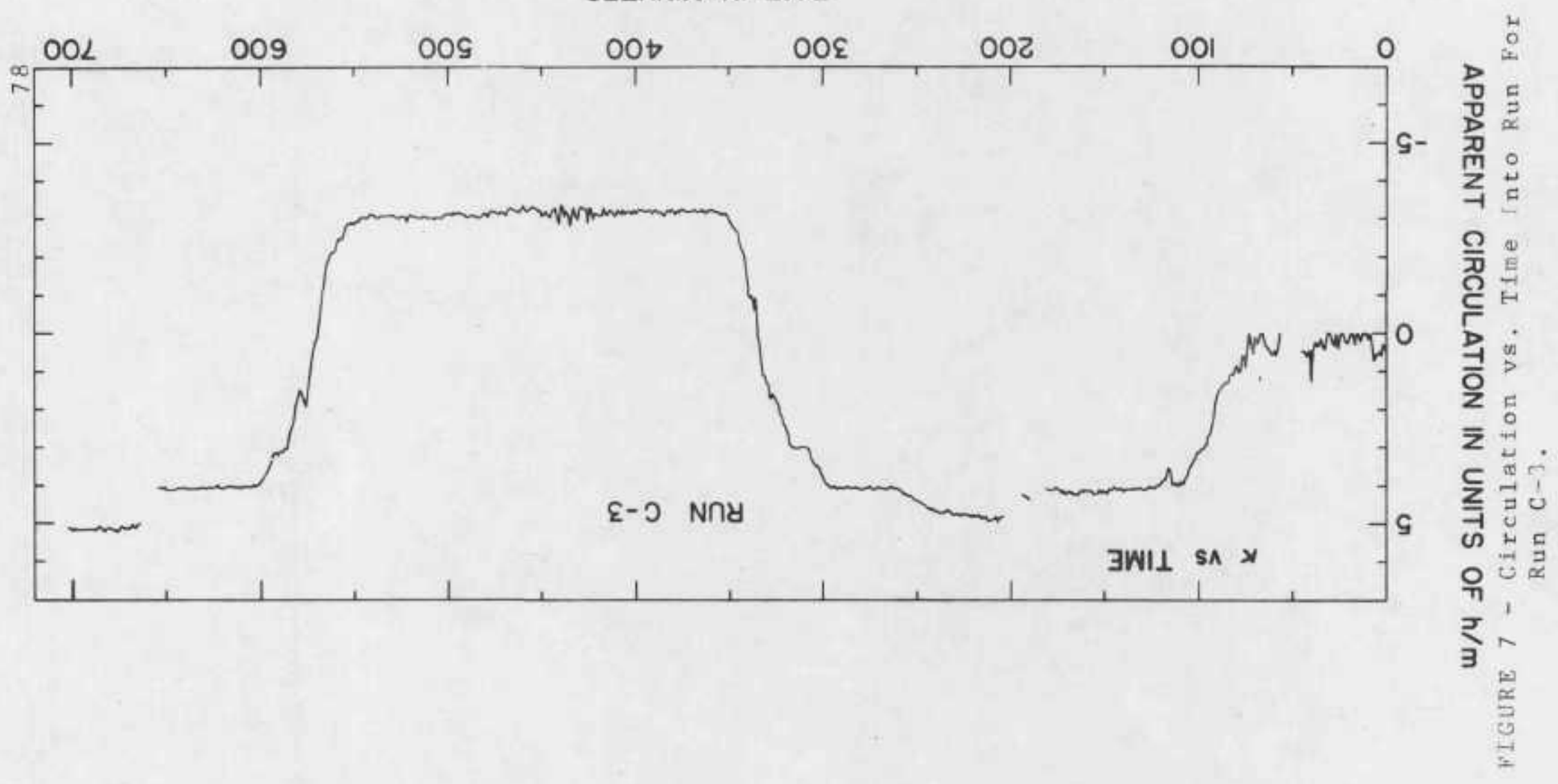




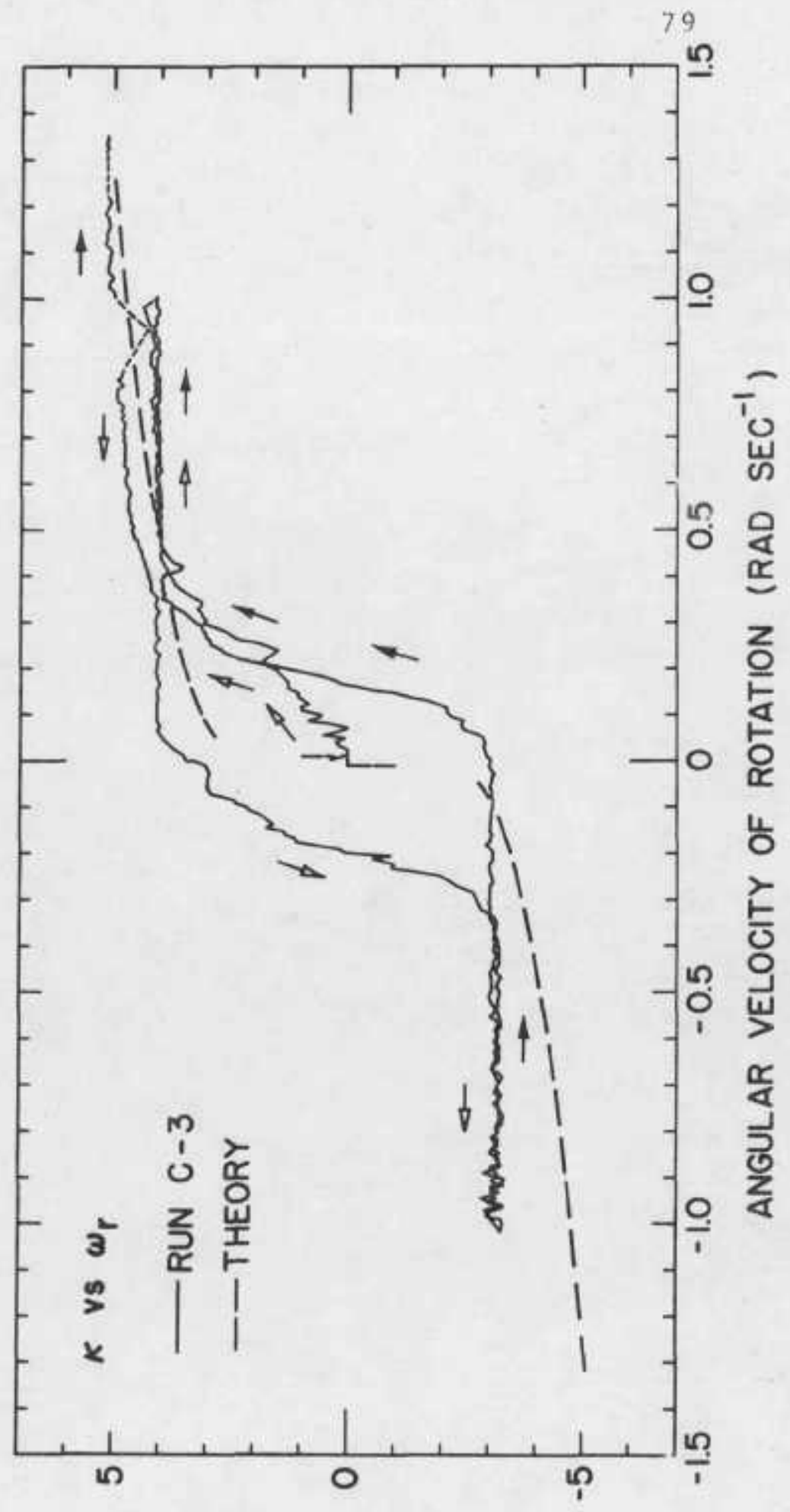

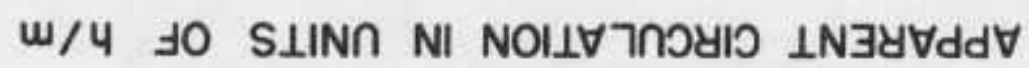

ILGURE 9 - Circulation vs. Angular Velocity of Rotation or Run $\mathrm{C}-3$. 


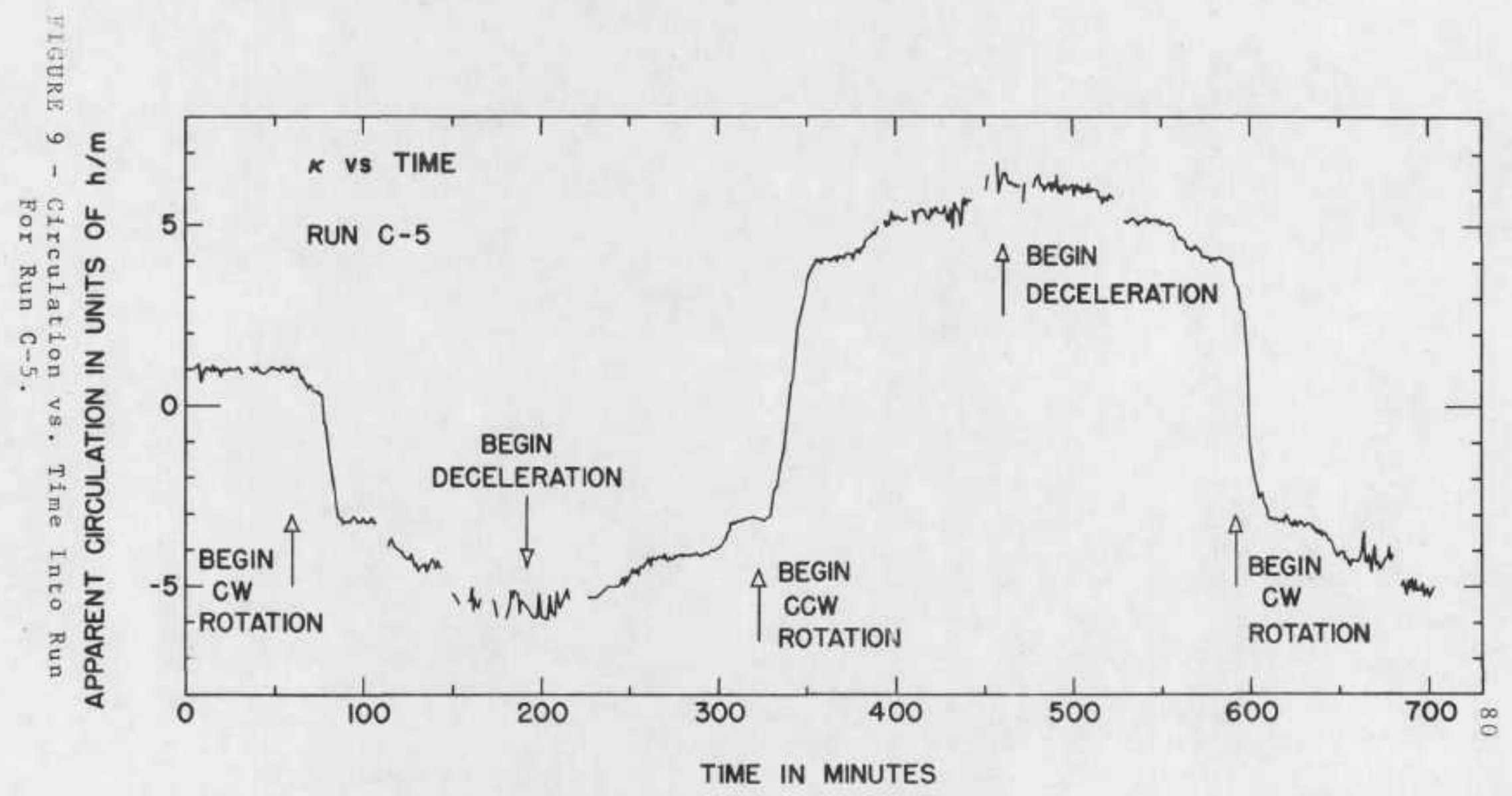




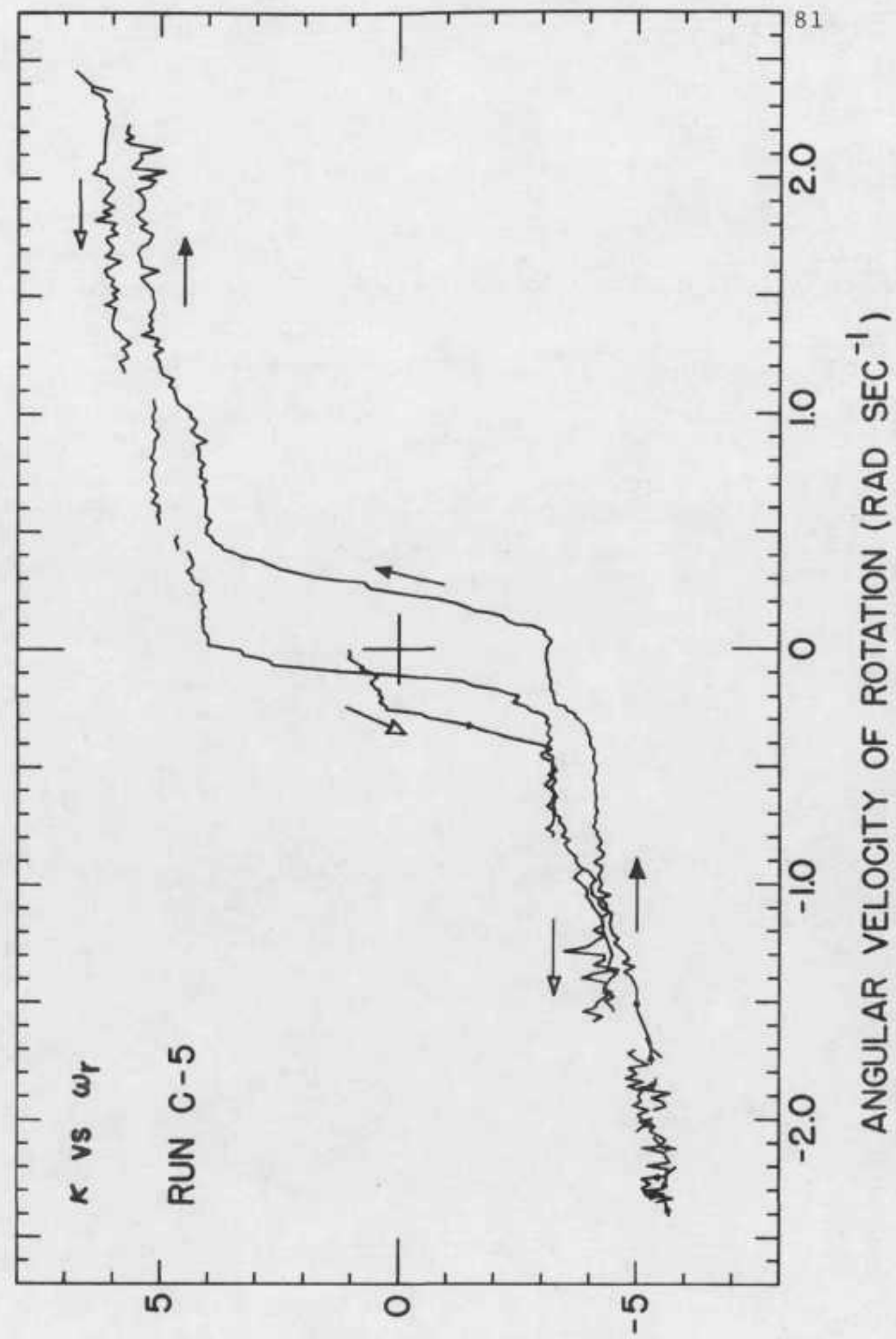

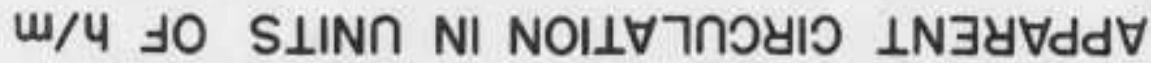

FIGURE 10 - Circulation vs. Angular Velocity Potation For Run C-5. 
values are plotted against rotation speed.

In all of the runs shown here it is apparent that the circulation is highly metastable, falling at both integral and non-integral multiples of the circulation quantum. This is to be contrasted with Whitmore's work in which the system showed selective metastability at integral multiples of the retio $\mathrm{h} / \mathrm{m}$. The causes of this general metastability are not clear. Behavior similar to that secn by Whitmore in the first few hours of his runs is observed in our system in the first few hours after the cell is filled. We see very unstable circulation during this period. However, twenty-four hours after the cell is filled, the circulation shows the unselective metastability mentioned above. This behavior is found when measurements are made both at rest and in rotation. Even after warming above $T_{\lambda}$ and cooling slowly at rest, the metastability at intermediate levels of circulation is seen. The determination of the circulation level is made more difficult by our inability to measure the intrinsic frequency splitting frequently and easily. However, it should be noted that it is not possible to bring all the circulation levels measured in a given run to 
integral multiples of the circulation quantum by a simple shift of the intrinsic frequency splitting. However, even in the presence of this metastability, certain features of the data are striking. The first principal result of our work is well illustrated in Fig. 5. The apparent circulation measured at a given rotation speed embraces 2 range of values, with the central value of this range increasing with increasing rotation speed. This is seen both in the runs made by making step-wise changes in the rotation speed and in the runs made with continuous acceleration. Except at small rotation speeds, the sense of the circulation is the same as that of the rotation.

Where, in the range of circulation values found at a given rotation speed, a circulation measurement will fall depends on how the rotation speed was reached. As is illustrated well in the runs with continuous acceleration, the lower values in the range are found when the system is accelerated to a rotation speed from a lower speed, and the higher values in the range are found when the rotation speed is reached by decelerating from a higher rotation speed. 
In addition, there appear to be limits to the range of metastable values the circulation may have at a given rotation speed. At very low rotation speeds and at rest the maximum magnitude of the circulation seen is near $3 \mathrm{~h} / \mathrm{m}$ in absolute value. In every case when the measured circulation in rotation equaled or exceeded the value $3 \mathrm{~h} / \mathrm{m}$, the circulation found when the system was brought to rest was very near $3 \mathrm{~h} / \mathrm{m}$. This observation strongly suggests the existence of an upper limit to the circulation at very low-rotation speeds. At higher rotation speeds the existence of an upper limit to the circulation is suggested by the fact that, even though circulation values exceeding $6 \mathrm{~h} / \mathrm{m}$ were seen at rotation speeds greater than $1 \mathrm{rad} / \mathrm{sec}$, when the system was decelerated the circulation was found to drop in steps eventually approaching the value $3 \mathrm{~h} / \mathrm{m}$. A lower limit to the circulation is suggested in Fig. 5. The portion of the loop traversed during the acceleration of the system from rest is seen to form a lower bound to the circulation for ${ }^{\omega}$ positive and an upper bound to the circulation for ${ }_{r}$ negative. With the exception of the region around zero rotation speed, no circulation measurements are found which 
lie outside these bounds.

2. Attempts to Change Circulation Levels

Many attempts were made to drive the circulation away from the metastable values intermediate to integral values toward integral values. While some attempts were successful in changing the circulation level, no method was found which would consistently drive the circulation to integral values.

A related problem was the rate at which the system would respond to changes in $\omega_{r}$. As can be seen from Tables Ia through Ic, most of the data were taken at temperatures near $1.3 \mathrm{~K}$. It was found that at these temperatures and at low rotation speeds the changes in circulation levels took place much more slowly than they did at higher rotation speeds. For example, at speeds greater than $0.5 \mathrm{rad} / \mathrm{sec}$ when the rotation speed was changed the system would move to a new circulation value in less than a minute. If, however, the system were accelerated from rest to a low-rotation speed, one less than $0.1 \mathrm{rad} / \mathrm{sec}$, it was found that for as long as two hours the circulation would drift towards its final metastable value before reaching it. Because of this slowness of response, great care had to be taken at low rotation speeds to assure that 
the circulation had reached a stable value.

After stability was achieved, attempts to estab$11 \mathrm{sh}$ new levels of stability at the system's rotation speed began. Following a method used by Vinen, the wire was driven repeatedly with large, short voltage pulses. (31) often the pulses would be so large that the wire's velocity amplitude would be as large as $200(\mathrm{~h} / 2 \pi \mathrm{ma})$. The results depended upon the rotation speed. At rest and at very low rotation speeds (1ess than $0.1 \mathrm{rad} / \mathrm{sec}$ ) it was found that these large pulses would often drive the circulation to some minimum value. The minimum value was not reproducible. On some occasions, further large pulses would increase the circulation from the minimum seen. If the circulation were changed by rotating the system and then bringing it back to rest, large pulses would again tend to reduce the circulation. The values obtalned, however, could not be related to the previous minimum values seen in any simple way, e.g., in a way which would suggest integral values of circulation. In rotation, for rotation speeds greater than $0.1 \mathrm{rad} / \mathrm{sec}$, when the system was pulsed with large pulses it was found that these pulses would change the circulation value, always reducing the measured 
circulation. Immediately after pulsing, however, the circulation would begin to increase from its reduced value and would return to its original value, or very near to it. The only exception to this behavior was when the sense of the circulation and the sense of the rotation were not the same. In this case after large pulses the circulation would usually have the same sense as the rotation. Unlike the changes induced at rest and at very $10 \mathrm{w}$ speeds, in rotation at higher speeds large permanentchanges in the circulation could not be Induced by large pulses.

In rotation another method was used which did bring about changes in the circulation. The circulation would be allowed to reach its metastable level at some rotation speed. Then the rotation would be Btopped and started as abruptiy as possible, usually by simply shutting off and later turning on the drive motor. An interval of one or two minutes between stopping and starting would usually be allowed. Using this method, it was found that if the circulation were near the lower limit of the thetastable region, it would increase, moving nearer the center of the region. On the other hand, if the circulation were near the upper limit of 
metastability, the circulation would be reduced, also moving nearer the center of the allowed range. The ultimate value the circulation took when this procedure was followed, however, was not, in genera1, an integral value.

\section{Runs With Constant Acceleration}

The second type of run in the experiment involved taking data while the system was being continuously accelerated. We note that the runs with continuously accelerated rotation lend further support to our statements concerning the limits of metastability. In particular, the higher circulation values seen at high rotation speeds are never seen at the lower rotation speeds, suggesting that they lie outside the limits of metastability at the lower speeds.

Runs in which the system underwent continuous acceleration enabled us to observe the phenomenon that is the second principal result of our work: when the system undergoes a continuous acceleration the circulation takes on stable values over a range of rotation speeds and often changes from stable value to stable value in step-like fashion. An hysteresis-1ike curve of circulation versus rotation speed is observed. This behavior is 111 ustrated in 
Figs. 5, 8, and 10. The step-like changes in the circulation are best seen when the system is rotated to speeds greater than $1 \mathrm{rad} / \mathrm{sec}$. Under these conditions with the wires we used, the circulation would rise above the lowest metastable level seen at rotation speeds greater than $0.2 \mathrm{rad} / \mathrm{sec}$ but less than $1.0 \mathrm{rad} / \mathrm{sec}$, which was near $3 \mathrm{~h} / \mathrm{m}$. Electrica1 noise becomes quite large at these rotation speeds and the scatter in the circulation data becomes quite large. However, the changes in the mean circulation from stable level to stable level in Figs. 7 and 9 are quite apparent. Further, the size of the step tends to be near one quantun of circulation even though the initfal and final values of the circulation are not at integer values.

Another interesting feature of the data is the point where the circulation curve crosses the ${ }^{\omega}{ }_{r}$ axis. The two wires used in continuous acceleration runs, wires $B$ and $C$, are both very similar in diameter. With these wires the crossing points in all runs fel1 at $\left|\omega_{\mathrm{r}}\right|=0.2 \pm 0.03 \mathrm{rad} / \mathrm{sec}$. 4. Errors

In estimating the uncertainty in the values of apparent circulation given in this thesis, we include effects arising from three separate sources. The 
first are the inherent instrumental resolutions which limit our ability to measure various quantities accurately. A second source is the systematic error which arises because a particular quantity which must be known well, the intrinsic frequencysplitting, could only be measured infrequently. A third source of uncertainty is that due to noise, particularly the electrical noise found in rotation which was somewhat dependent on the rotation speed. The first and third sources are similar in nature but we wish to emphasize the problem of rotational electrical noise. In computing the apparent circulation in units of h/m, Eq. $2-73$ was solved yielding

$$
\mathrm{n}=\left(\frac{\mathrm{h}}{\mathrm{m}}\right)^{-1} \mu / \rho_{\mathrm{s}}\left[\Delta \omega_{\mathrm{r}} \pm \sqrt{\left(\Delta \omega_{\mathrm{t}}\right)^{2}-\left(\Delta \omega_{0}\right)^{2}}\right] \cdot 4-1
$$

We must estimate the error in each of the quantities entering into this expression. Then we will combine them to find the uncertainty in the apparent circulation.

\section{Errors in Measured Quantities}

The wire's effective mass per unit length $\mu$ was calculated by adding the wire's measured mass per unit length and the mass per unft length of normal fluid and superfluid displaced by a cylinder of 
radius equal to the mean radius a of the fiber,

$$
\mu=\frac{m_{W}}{L_{W}}+\pi a^{2}\left(p_{s}+p_{n}\right) \cdot \quad 4-2
$$

We approximated the constant $K$ by unity. In addition, the wire's ellipticity has been ignored.

The wire's mass m was measured to an accuracy of $+2 \mu g$, which for wire A, yields a percentage error in the mass of $0.45 \%$, for wire $B, 0.25 \%$, and for wire $C, 0.25 \%$. The wire's length, $L_{W}$, was measured to an accuracy of $\pm 0.025 \mathrm{~cm}$. For each of the three wires this error yields a percentage error in the length of $0.41 \%$. Perpendicular diameters of the wires were measured; for wires $A$ and $C$ they were found to be within $6 \%$ of each other, while for wire B they were within $0.5 \%$ of each other. The quantity $P_{S}$ is known to about $1 \%$ at the temperature at which the experiment was done, the value being obtained by comparing the values of Reynolds, et, al. (31) with the values obtained by Dash and Taylor. (32) The constant $K$, which was approximated by 1 was actually about 1,6. Each of these uncertainties contributes to a total uncertainty in the effective mass per unit length of the wire through the expression 


$$
\begin{aligned}
\frac{\delta \mu}{\mu}= & \frac{1}{H}\left[\frac{1}{L_{w}}\left(\delta m_{w}+\frac{m_{w}}{L_{w}} \delta L_{w}\right)+2 \pi a \delta a\left(\rho_{s}+\rho_{n}\right)\right. \\
& \left.+\pi a^{2}\left(\delta \rho_{s}+K \delta \rho_{n}+\rho_{n} \delta K\right)\right] .
\end{aligned}
$$

This expression yields the fractional error in the effective mass per unit length of wire A as $1.78 \%$, of wire $B$ as $0.83 \%$, and of wire $C$ as $1.5 \%$. Since $\frac{\delta n}{n}$ is equal to $\frac{\delta \mu}{\mu}$ (ignoring other errors) we have that due to errors in determining the mass the fractional error in $\mathrm{n}$ is $1.78 \%$ for wire $\mathrm{A}, 0.83 \mathrm{z}$ for wire B, and $1.5 \%$ for wire C.

The error in the superfluid density also enters in a direct way, and ignoring other errors, we have $\frac{\delta \mathrm{n}}{\mathrm{n}}=\frac{\delta \rho_{\mathrm{s}}}{\rho_{\mathrm{s}}}$ which is $1 \%$ for all three wires. For the runs made with fixed rotation speed the percentage error in $\omega \mathrm{r}$, as estimated from the scatter in the measurements of the time for a single rotation, is, in maximum value, $0.5 \%$. The times obtained for a single rotation when the system was being accelerated were fittcd to an analytic expression. The criterion for a good fit was that the value for the time for a single rotation computed from the function should agree with that measured to $1 \%$ or better. The error in $\omega_{\mathrm{r}}$ was 
taken to be $1 \%$. The error in $n$ then, ignoring other errors is given by

$$
\delta \mathrm{n}=\left(\frac{\mathrm{h}}{\mathrm{m}}\right)^{-1} \mu / \rho_{s} \delta\left(\Delta \omega_{\mathrm{r}}\right) . \quad 4-4
$$

The 1 imits of error in $n$ for the range of rotation speeds covered with wire $A$ are $0 \leq \delta n \leq 0.04$; for wire $B$ the $11 \mathrm{mits}$ of error are $0 \leq \delta \mathrm{n} \leq 0.02$, and for wire $c$ the 1 imits of error are $0 \leq \delta \mathrm{n} \leq 0.05$.

The measured time to the first node of the wire $t_{n}$, has contributions to its error from the following: uncertainties in the time delay due to use of the tuned amplifier, our fallure to correct for the finite length of time of the drive, and uncertainties arising from the inability to resolve the node position exactly, which is related to the calibration of the time sweep of the oscilloscope.

The tuned amplifier introduces a delay in the appearance of the node. This delay $t_{D}$ is given by $t_{D} \simeq \frac{1}{\gamma-\lambda}$ where $1 / \gamma$ and $1 / \lambda$ are the time constants for free decay of the tuned amplifier and the wire, respectively. These time constants are obtained from halving-time measurements made on a signal 
observed on the graticle of an oscilloscope whose sweep speed was callbrated. The error in the estimate of the halving time was taken to be $1 \mathrm{~mm}$ of the graticle, which contributed an absolute error of $0.1 \mathrm{msec}$ for the amplifier halving time and 2 msec for the wire's halving time. The percentage error in the calculated time delay wes then $3 \%$, for typical experimental values of $\gamma$ and $\lambda$. Since the expression used for $t_{D}$ is an estimate good to $1 \%$ accuracy, we take the total error in the delay to be $4 \%$.

The error introduced because of the finite length of the drive is mentioned in Chapter II. Since, while the wire is being driven, the plane of the wire's oscillation only precesses about one half the distance it would if the wire were vibrating freely, a correction fos this effect must in principle be introduced. In practice the circulation was calculated as if the plane did not begin to rotate until after the drive was shut off. As a consequence, an error of some $4 \mathrm{msec}$ was introduced. The length of the drive time itself was read from the screen of the oscilloscope with the calibrated graticle, with an error of $0.1 \mathrm{msec}$. 
The error in the measurement of the time at which the node occurred depends upon the accuracy of the automatic data-taking system. However, in practice, the times recorded by the automatic datataking system were always checked against visual observations on the calibrated oscilloscope. Thus, the primary uncertainty in measuring the node time was that introduced by the limit of resolution of the graticle, $0.5 \mathrm{~mm}$. This corresponded to an error of $5 \mathrm{msec}$, for the sweep speed used.

The most important source of uncertainty in our knowledge of the time to the node caine about because of electrical noise. As can be seen in Fig. 6, the scatter in the time to the node became very large when the system was rotating rapidiy. Thls scatter was the result of electrical noise generated in the slip rings of our rotating electrical connection. This noise took the form of short current pulses. In themselves, they were too short in duration to cause a problem. However, they did cause ringing in the tuned amplifier, and if the amplifier was ringing when the node occurred, the node would be obscured. An estimate of the error caused by this effect can be made from the amount of scatter in the 
node times. This error is dependent on rotation speed, ranging from $\pm 15 \mathrm{msec}$ at the 1 arger rotation speeds to less than a millisecond for the smaller rotation speeds. The larger value appears applicable to $\omega_{r}>0.5 \mathrm{rad} / \mathrm{sec}$ with the smaller value applying below that.

The error in $\Delta \omega_{t}$ which arises from these sources can be found from the expression $\Delta \omega_{t}=\pi / t_{\text {node }}^{\text {corrected }}$ where $t_{\text {node }}^{\text {corrected }}=t_{\text {node }}^{\text {measured }}-t_{\text {drive }}-t_{\text {delay }}$.

We note that $\Delta \omega_{0}$ is found from measurements of the maximum node time observed in the course of the run, which we call $t_{0}$. This node tiae measurement is also subject to the errors discussed above. The quantity $\Delta \omega_{0}$ is obtained from the expression $\Delta \omega_{0}=$ $\pi / t_{0}^{\text {corrected }}$, where $t_{0}^{\text {corrected }}=t_{0}^{\text {measured }}-t_{\text {drive }}$

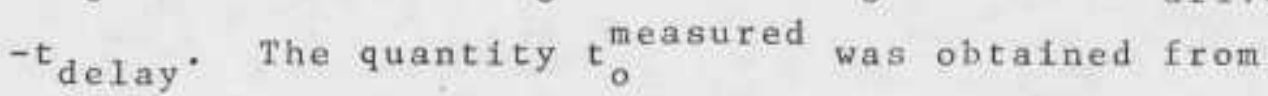
those few times in the course of a run the time to the node achieved its maximum value and was reproducible to within $8 \mathrm{msec}$. This limit of reproducIbility was taken to be the uncertainty in $t_{0}^{\text {measured }}$. The error in $n$ due to the error in $t$ measured is given by

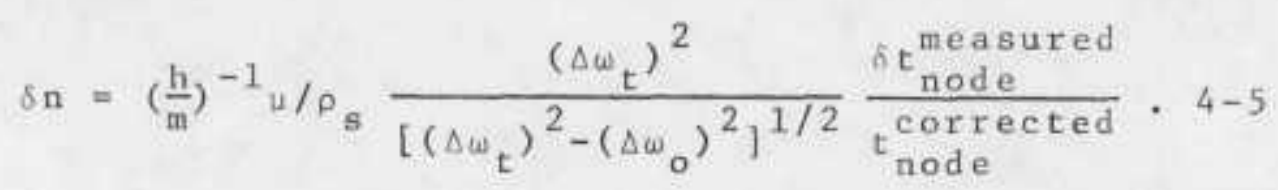


The error in the circulation values obtained with wire A are computed using the larger limit of error in $t_{\text {node }}^{\text {measured }}$, since most of these measurements were made at high rotation speeds. The error is found to be $\delta n=0.76$ over almost the entire range of rotation speeds used. For wires $B$ and $C$ the typical errors in n are very similar. At high rotation speeds the error in the circulation value is found to be $\delta \mathrm{n}=0.23$, while at low rotation speeds the erior in the circulation values is $\delta \mathrm{n}=0.07$. These errors are calculated for typical node times giving rise to a circulation value near to 3 quantum units. At higher circulation levels the error in $\mathrm{n}$ becomes as large as $\mathrm{n}_{\mathrm{n}}=0.4$. The error in $n$ due to errors in $t_{0}^{\text {measured }}$ is given by an expression similar to Eq. 4-5. For wire A the error in $n$ is found to be $\delta n=0.33$, the large absolute error being due to the larger value of $\Delta w_{0}$ used with this wire. For wires $B$ and $C$ the error in $n$ is found to be $\delta n=0.1$ for values of $n$ near to 3 quanta of circulation.

Finally we note that errors arising because of the time delay and our failure to correct for the finite length of drive enter into both the calculation of $\Delta \omega_{t}$ and $\Delta \omega_{0}$. The error introduced into $n$ because of the delay time takes the form 


$$
\begin{aligned}
\delta \mathrm{n} & =\left(\frac{\mathrm{h}}{\mathrm{m}}\right)^{-1} \mu / \rho_{s} \frac{\left(\Delta \omega_{\mathrm{t}}\right)^{2}}{\left[\left(\Delta \omega_{t}\right)^{2}-\left(\Delta \omega_{0}\right)^{2}\right]^{1 / 2}} \delta t_{\text {delay }} \\
& \times\left[\left(\frac{\Delta \omega_{0}}{\Delta \omega_{t}}\right)^{2} \frac{1}{t_{0}^{\text {corrected }}}-\frac{1}{t_{0}^{\text {corrected }} \text { node }}\right] \cdot \quad 4-6
\end{aligned}
$$

For wire A the absolute error in $n$ due to the delay time is found to be $\delta \mathrm{n}=0.08$. For wires $\mathrm{B}$ and $\mathrm{C}$ this error is found to be $\delta n=0.01$.

The error introduced because of our fallure to correct for the finite drive time takes a form similar to Eq. 4-6. For wire A this error is found to be $\delta \mathrm{n}$ $=0.16$ and for wires $B$ and $C$ it is found to be $\delta n=$ 0.10 .

To find the combined error we compute the standard error for each wire. (34) For wire A we find the error in the circulation value to be as large as $\delta \mathrm{n}=0.85$ at the highest rotation speeds and $\delta \mathrm{n}=0.46$ at the lower rotation speeds. These errors are quoted for values of n near to five quanta of circulation. For wires $B$ and $C$ we find the standard error to be very similar for both wires. The standard error is found to range from on $=0.16$ at the lowest rotation speeds to $\delta \mathrm{n}=0.43$ at the highest rotation speeds. For three quanta of circulation, which was a typical value of $n$ found with these wires, the standard error is found to be $\delta \mathrm{n}=0.27$. 


\section{CHAPTER V}

THEORY AND DISCUSSION

\section{Equilibrium Theory}

The equilibrium theory of rotating superfluid helium is based upon the minimization of the freeenergy of the rotating superfluid given by

$$
F=E-L w_{r}, \quad 5-1
$$

where $\mathrm{B}$ is the energy of the superfluid, L is the angular momentum of the superfluid, and $\omega_{r}$ is the angular velocity of rotation. (35) This minimization must be carried out with the constraints imposed by the possible presence of quantum vortices in the fluid and by the possibility of quantized circulation around solid obstacles forming internal boundaries in the fluid. (36)

If we consider a rotating annulus whose outer radius $\mathrm{R}_{2}$ is much greater than the inner radius $\mathrm{R}_{1}$, the following results are found. (37) If the superfluid is inftially stationary, it will remain stationary until a certain critical rotational velocity ${ }_{r 1}$ is reached. At this velocity, given by

$$
{ }_{\mathrm{r} 1}=\frac{\mathrm{h}}{\mathrm{m}\left(2 \pi \mathrm{R}_{2}^{2}\right)} \ln \left(\mathrm{R}_{2} / \mathrm{R}_{1}\right), \quad 5-2
$$


it becomes energetically favorable for a single quantum of circulation to form around the center cylinder. This angular velocity is the rotational velocity indicated by the dashed vertical line in Fig. 8, which for wire $C$ is about $0.010 \mathrm{rad} / \mathrm{sec}$. As the rotation speed increased, the single quantum of circulation remains around the center cylinder until a second critical velocity given by ${ }^{\omega}{ }_{2}={ }^{3 \omega}{ }_{\mathrm{r} 1}$ is reached, at which a second quantim of circulation is formed around the inner cylinder. Further increase in the rotation speed results in creation of vortices in the fluid as well as higher levels of circulation around the inner cylinder, the precise sequence of events depending upon the size of the inner and outer radif and the vortex core parameter.

In the limit when many vortices are present in the fluid, it is possible to treat the problem of the equilibrium rotating superfluid flow in the continuum approximation. (38) In this approximation one assumes that the discrete vortex structure may be replaced by a smoothed vortex density. This approximation is essentially a classical limit, in which Planck's constant h approaches zero with the circulation enclosed by any contour in the fluid $\mathrm{n} \mathrm{h} / \mathrm{m}$ remaining finite. When this approximation is 
applied to a rotating annulus, the following results are found, upon minimization of the free-energy:

(1) The equilibrium vortex density $n$ is uniform throughout the fluid and is given by

$$
\mathrm{n}=2 \omega_{\mathrm{r}}(\mathrm{m} / \mathrm{h}) . \quad 5-3
$$

(2) The equilibrium circulation around the inner cylinder of the annulus, of cross-sectional area $A$, is given by

$$
\kappa_{\text {eq }}=2 \omega_{\mathrm{r}} \mathrm{A} \text {. }
$$

Thus, although the fluid remains irrotational at every point except at vortex cores, the macroscopic flow pattern is indistingulshable from uniform rotation in which $|\operatorname{cur} 1 \vec{v}|=2 \omega_{r}$.

An extension of this approximation has been proposed in which an attempt is made to take into account quantum effects. ( 39 ) In this extension, in the $11 \mathrm{~m} 1 \mathrm{t}$ of many vortices, the free energy is written approximately as

$$
F=F_{c l}+A n\left(\rho h^{2} / 4 \pi m^{2}\right) \ln (b / a), \quad 5-6
$$

where A is the area occupied by the vortices of density $\mathrm{n}$, a is the vortex core parameter, and $\mathrm{b}$ is a length comparable to the mean vortex spacing given by $s=(n)^{-1 / 2}$. $F_{c l}$ is the free energy of a classical liquid whose velocity field is equal to the mean 
velocity of the vortex system averaged over regions containing many vortices. The second term is the self-energy of the vortices.

Using this approximation to find the distribution of vortices in a rotating cylinder containing He II, it is found that the vortices are unformly distributed throughout the cylinder, except for a narrow vortex-free strip near the walls of the cylinder. The presence of this vorlex-free strip near the outer wall tends to reduce the free energy without significantly affecting the solid body velocity field. In an annulus this vortex-free strip is present near both the inner and outer walls, but the reduction in the mean velocity due to the missing vortices must be compensated for by additional circulation around the inner cylinder. In such a system the mean velocity field is, by symmetry, azimuthal, and may be written

$$
\begin{array}{lll}
v(r)=k / 2 \pi r & R_{1} \leq r \leq R_{1}, & 5-7 a \\
v(r)=\omega_{r} \cdot r & R_{1} \leq r \leq R_{2}, & 5-7 b
\end{array}
$$

where $R_{1}$ and $R_{2}$ are the inner and outer radif of the annulus and $k$ is the circulation around the core of the annulus. The radius $R_{1}$ is the radius of the region around the inner boundary of the annulus from 
which vortices are excluded. We determine $R_{i}$ by insisting that the mean velocity field be continuous at $r=R_{1}$. We have ignored the effect of the vortexfree strip near the outer wall; its presence has no effect on our results. In a unit length along the axis of rotation we have

$$
F=E_{c \ell}-L_{c \ell} \omega_{r}+n A\left(P_{s} / 4 \pi\right)\left(\frac{h}{m}\right)^{2} \ln \left(\frac{b}{a}\right), \quad 5-8
$$

where

$$
E_{c l}=\frac{1}{2} P_{s} \int(v(r))^{2} d^{2} r \text {, }
$$

and

$$
L_{c \ell}=\rho_{s} \int(v(r)) \cdot r d^{2} r .
$$

Evaluating $F$ we find

$$
\begin{aligned}
F=\rho_{s} k^{2} / 4 \pi\left[\frac{1}{2} \ln \frac{k}{k_{c l}}-\frac{3}{4}+\frac{k_{c l}}{k}\right]-\frac{\pi \rho_{s}{ }^{\omega}{ }_{r}^{2} R_{2}^{4}}{4} \\
+\frac{\rho_{s}}{4 \pi}\left(\frac{h}{m}\right) \ln \frac{b}{a}\left(2 \pi \omega_{r} R_{2}^{2}-k\right), \quad 5-11
\end{aligned}
$$

where $k_{c l}$ is given by $2 \pi \mathrm{R}_{1}^{2}{ }^{\omega}{ }_{r}$. We wish to find a stationary value of $F$ with respect to variations in $k$, i.e., the values of $k$ which yield $a F /\left.\partial K\right|_{\omega_{r}}=0$. We ignore the quantization of circulation in this calculation. Treating $k$ as a continuous variable, we find the values of $k$ to be given by the expression

$$
k_{c \ell}-k\left(1-\ell n \frac{k}{k_{c \ell}}\right)=\frac{h}{m}\left(\ell n \frac{b}{a}\right) \cdot 5-12
$$


This expression gives the equilibrium value of

$k$ for any rotation speed. In evaluating

Eq. 5-12, we use the value 0.27 s for b obtained from a rigorous calculation assuming a triangular latice structure. $(40)$

In obtaining Eq. 5-12 from Eq. 5-6, we have treated $k$ as a continuous variable. The equilibrium circulation at a given rotation speed is expected to be that integral multiple of the circulation quantum which produces a local minimum in the free energy. This value can be found by taking the integral multiples of $\mathrm{h} / \mathrm{m}$ nearest to the value of $K$ predicted by Bq. 5-12 and determining which of these corresponds to the smallest value of the free energy, F. The circulation value producing the smallest free energy under these conditions is presumably the equilibrium circulation.

In Fig. 4 the curve of the equilibrium circulation for wire $A$ is plotted along with the data obtained from runs with wire A.

A similar curve is plotted in Pig. 8, superimposed on the results of run $\mathrm{C}-3$. On the same figure from run $\mathrm{C}-3$ is indicated the critical 
velocity at which it becomes favorable to form a single quantum of circulation in the cylindrical annulus defined by the wire and containing cylinder, if this annulus is accelerated from rest in a vortex and circulation-free state. We see from these figures that the circulation values corresponding to the center of the allowed region are described wcll by the equilibrium values predicted by this simple theory. Furthermore, the results of this theory depend upon the quantization of circulation through the term on the right-hand side of Eq. 5-12. Thus the agreement between our results and this theory gives indirect support to the principle of quantization of circulation.

It is of interest that recent experiments have tended to verify other features of this theory. Northby and Donneliy, using fon measurements, have shown that there is a vortex-free strip near the outer wall of the rotating annulus, although their results yield a value for the strip-width larger than predicted. (43) Shenk and Mehl, using second sound attenuation in a rotating annulus, have shown good agreement with the prediction that there is a vortex-free strip near the inner and outer wal1. (44) It would also be interesting to study the 
circulation around the wire as the annulus is accelerated from zero rotation speed in a vortexfree, circulation-free state. This experiment is within the capability of our apparatus. Unfortunately, our method of filling the cell apparently precluded the formation of such a state.

\section{Discussion}

The work presented here extends the experiments of Vinen and of Whitmore and Zimmermann. Using the technique originated by Vinen, we have eztended Vinen's measurements of the circulation in the rotating annulus.

The results of our work are not inconsistent with the earlier work. The metastability of the circulation which is such a prominent feature of our work is consistent with the trend of Whitmore's data. In Whitmore's work the c1rculation becane more and more stable as time progressed. While Whitmore did observe this greater stability during the later hours of his runs, he was unable to measure circulations for times exceeding 24 hours, and so his data did not extend into the time regime of our work. While the behavior of the circulation we observe for the first few hours after the filling of our cell agrees with Whitmore's observations, we 
have no data during the crucial twelve-hour period during which Whitmore saw the highly selective metastable behavior which characterized his results. Certainly any further work with our system should include studies during this time period.

An interesting parallel between the measurements made at rest by Whitmore and our measurements at rest is found in the maximum values of circulation seen. In Whitmore's work with his largest wires, the stable circulation observed was at the 3 quantum level. Our observation that we always see a circulation of magnitude near to 3 quantum units when we come to rest after rotating at a rotation speed greater than $0.2 \mathrm{rad} / \mathrm{sec}$ is consistent with his result. Our wires and his largest wires were of comparable size.

The feature of our results that is difficult to understand is the unselective nature of the metastability observed. The amounts by which the circulation values differ from integral values appear to be outside the estimated range of error for the experiment. The remarkable stability the circulation shows over a fairly wide range of rotation speeds does suggest that a stable hydrodynamic flow around the wire is present. In F1g. 5, for example, 
we see very stable circulation over long periods of time even though the angular velocity of the system was undergoing large changes. The same behavior is seen in F1g. 8. Further, many, though by no means a11, of the steps through which the circulation goes upon acceleration and deceleration appear to be very nearly one quantum unit in size, albait between non-integral circulation values.

It is interesting to note that when the system is accelerated from zero rotation speed starting with a low circulation value, less than 1 quantum, no steps are seen at the 1 and 2 quantum levels. On the other hand, when the system is decelerated from a high rotation speed, the circulation drops in step-like fashion towards 3 quantum units. When the system is accelerated with opposite sense, the change in circulation, as it diminishes to zero and reestablishes itself with the opposite sense, does not always take place smoothly. In Fig. 5, for example, the curve shows a shoulder between the 1 and 2 quantum levels and between the -1 and -2 quantum levels. This behavior has been observed in other runs, but as can be seen in Fig. 10, it is not always observed. Why above the 3 quantum level we always see step-like behavior and below it we 
only sometimes see a suggestion of it is not clear. We have made these observations in rotation with angular accelerations extending from $2.3 \times 10^{-4}$ $\mathrm{rad} / \mathrm{sec}^{2}$ to $3.44 \times 10^{-5} \mathrm{rad} / \mathrm{sec}^{2}$.

A possible explanation for the unselective metastability we observe is suggested in a paper by Griffiths. (45) In that work, estimates are given for the stability of a vortex pinned to an irregularity on the wire and extending to the wall of the outer cylinder. As we noted in Chapter II, a singly quantized vortex pinned in this way would give rise to a difference of one quantum unit in the circulation of the fluid around the wire above and below it. Since we measure an average circulation along the length of the wire, a pinned vortex could explain a non-1ntegral circulation measurement. A result of Griffith's work is that for large values of circulation around the wire (values greater than 2 quantum units) a stable configuration of a vortex pinned to the wire is not possible for the system considered. This conclusion is based on assumptions about the details of the pinning process and on the size of the surface irregularities.

The general agreement between the circulation values measured here and the predictions of the 
equilibrium theory suggest that further studies be made, particularly with smaller wires. W1th sma11er wires the theory predicts that smaller circulation values would be seen at a glven rotation speed. With smaller wires the predicted regions where zero and one quantum of circulation represent the equilibrium state would be more easily accessible. With smaller wires we would also expect the width of the metastable region to be reduced. The slze of the metastable region we observe at low rotation speeds just covers the range of circulation values Whitmore observed with wires of similar diameter at rest. (46) With wires of smaller diameter Whitmore observed a smaller range of circulation values; we would expect a correspondingly narrower metastable region. Finally, with smallex wires, Griffith's analysis suggests that vortex pinning might not be a serious problem.

It would also be useful to take data at lower temperatures. In Whitmore's work, the evidence for selective metastability appears to be more clear cut at his lowest running temperature than at his highest temperature. Also, the circulation changes which he observed seemed to occur more rapidiy at lower temperatures than at higher temperatures. 
APPENDIX I

EXPERIMENTAL APPARATUS

\section{Introduction}

The apparatus used in this experiment was designed so that the circulation of the superfluid in the sample chamber could be measured under as wide a range of temperatures and pressures as possible, both in rotation and at rest. To this end, an apparatus was built capable of operation at rest or in rotation, with the pressure of the helium in the sample chamber ranging from the saturated vapor pressure to the solidification pressure, and at temperatures to as low as approximately $0.4 \mathrm{~K}$. In addition to these requirements, the apparatus had to meet other standards related to the performance of the wire itself, such as vibration isolation, provision for wire frequency adjustment, and wire frequency stability.

\section{Overall Apparatus}

An overall drawing of the apparatus is shown in Fig. 1. The liquid helium and liquid nitrogen dewars are supported by a stand made of welded 2 in. aluminum square tubing. The dewar stand rests upon an aluminum plate which is vibrationally 
isolated from most floor vibrations by means of coil spring shock mounts. By loading the dewar stand with lead bricks, the vibration frequency of the dewar stand system is reduced to only a few cycles per second. Since the wire frequency itself is typically around 500 cycles per second and the wire has a large mechanical Q, the vibration isolation system is sufficient to isolate the wire from most building vibrations. In addition, further effects of building nolse were reduced by making most measurements at night.

The apparatus itself rested upon a turntable so that it could be easily rotated. The turatable rode on a 5 in. diameter aircraft ball bearing. Inside the ball bearing a commercial ofl seal provided a vacuum seal between the turntable and the liquid helium dewar so that the helium dewar could be pumped, as was necessary in our set-up runs. Both the ofl seal and bearing required frequent attention to insure smooth rotation.

The turntable was driven by a motor drive (Graham type N30VM) whose speed is continuously variable. This motor drive was bolted to the floor. Under most running conditions the output of the motor drive was further reduced by a factor of 25 by a 
gear reduction box (Boston Gear type TW109-25). With the output of the gear box driving a 3 in. diameter pulley, and with this pulley driving the $12 \mathrm{in}$. dlameter turntable pulley by means of two $1 / 4 \mathrm{in}$. "O" rings, it was found that the turntable could be rotated over a range of speeds from 0 to $3 \mathrm{rad} / \mathrm{sec}$. The coupling between the motor-gear-reduction-system and the turntable was made using rubber connectors and sylphon bellows as vibration isolators. When the system was running at top speed and turning the $3 \mathrm{in}$. diameter pulley, but not the turntable itself, it was found that the wire noise level was not more than $10 \%$ greater than the level with the motor off. This is a measure of the vibration transmitted to the wire by the couplings between the motor and the dewar stand. Direct attempts to measure the mechanical noise when the apparatus was in rotation proved frultless since the electrical noise present during rotation masked the mechanical noise. Indirect attempts, such as listening with ear pressed to dewar stand, or watching the surface of water standing in a beaker and rotating with the turntable, suggested that the mechanical noise present during the rotation was not significant 1 y larger than that present in the absence of rotation. 
The motor drive speed was varied in two ways. During early runs the speed was changed manually, in step-like fashion. During later runs the motor drive speed control was driven continuously by a second motor (Bodine motor type NSH 12-R), and, except at very low speeds, the angular acceleration was constant.

Careful alignment of the apparatus was essential to guarantee smooth rotation. The $2.54 \mathrm{~cm}$ external $\mathrm{He}^{4}$ pot pumping line was the source of most of our difficulty in this respect. Since the clearance between the magnet and the dewar wal1 was on $1 \mathrm{y} 3.13 \mathrm{~mm}$, a slight misalignment of the $\mathrm{He}^{4}$ pot pumping line rotating pumping connection (see Fig. 1) with respect to the rotation axis would cause the apparatus to rub at the lower end. This alignment was usually checked at room temperature before cool-down. Another possible source of vibration was the He ${ }^{4}$ pot pumping line. Vibration isolation was achieved by using a one-foot length of flexible stainless steel tubing. This method was not totally successful, since it was found that when the $\mathrm{He}^{4}$ pot was pumped by a pump located in the room, some of the pump's vibration would be coupled to the apparatus by the pumping line. A more successful means of pumping, 
and that normally used, was to use a remote pump (Stokes model 149-80CFM) located elsewhere in the building and connected to the He $e^{4}$ pot pumping line by a $7.64 \mathrm{~cm}$ diameter line.

When the apparatus was at rest, the $\mathrm{He}^{3}$ pot could be pumped using a special valve with a demountable valve operator (Cryolab model SVI-88-251). This pot was pumped through a $5.08 \mathrm{~cm}$ diameter external ine, which had a length of flexible stainless steel tubing along its length. This means of vibration isolation was not totally successful. However, in the work described here we did not use this pumping line, and consequently we were not disturbed by the vibration.

After all the precautions mentioned above were taken, it was found that the main mechanical noise sources were the bolling of the liquid nitrogen and noise transported through the air from various noise sources in the room, such as vacuum pumps and fans. Since it was necessary to have a few pumps operating in the room while data was being taken, nolse-absorbing boxes were built around all the pumps which were running. The noise level in the room and the noise directly coupled through the dewar stand were small enough that the wire vibrations excited by voices could be seen easily. 
Additional features of the overall apparatus not shown in Pig. 1 were a $\mathrm{He}^{3}$ gas handling system used to collect and recirculate $\mathrm{He}^{3}$, and a $\mathrm{He}^{4} \mathrm{gas}$-handling system which could be used to fill the sample chamber and control the pressure of the vapor above the liquid in the sample chamber in a range from the pressure of the saturated vapor to the solidification pressure.

\section{Cryogente Features of the Apparatus}

Figure 3 is a schematic drawing of the apparatus below the vacuum can flange. In a typical run, this part of the apparatus would be immeraed in a bath of helium at $4.2 \mathrm{~K}$. Because the tension-control assembly, electrical leads, and fill capillaries were al1 thermally grounded at the vacuum can flange, and had to be kept at $4.2 \mathrm{~K}$, It was necessary to keep the vacuum can flange at that temperature throughout the run. This was done first by fllifing the dewar well over the level of the flange. During the course of a long run, the helium level would fall below the flange. In order to make sure the flange remained at $4.2 \mathrm{~K}$ even under this condition, the vacuum can was made of copper so as to assure good thermal contact between flange and helium. The maximum practical time between transfers of helium into the $4.2 \mathrm{~K}$ bath was 18 hours. For 
timeslonger than this the temperature of the flange would begin to rise, giving rise to undesirable heat leaks into the vacuum can.

4. The He ${ }^{4}$ Pot

The $\mathrm{He}^{4}$ pot had a volume of approximately $110 \mathrm{~cm}^{3}$. To inhibit film flow a $0.75 \mathrm{~mm}$ diameter orifice was placed at the pumping outlet of the pot. Above the orifice, up to the vacuum can flange, the pot was pumped through a $0.471 \mathrm{~cm}$ diameter stainless steel tube. Above the vacuum can flange the pumping line was $0.795 \mathrm{~cm}$ in diameter. This tube led, at room temperature, to the $2.54 \mathrm{~cm}$ diameter pumping line mentioned before. The $2.54 \mathrm{~cm}$ lint at room temperature was stationary at a11 times. A special rotating pumping seal consisting of commercial oil seals backed by " $O$ " rings was used to make connections between the stationary line and the rotating apparatus. Leak testing showed this seal to be leak-tight under al1 operating conditions.

The copper walls of the He $e^{4}$ pot also acted as a thermal ground for every element entering the vacuum can from $4.2 \mathrm{~K}$. The electrical leads were varnished to the walls of the pot. The sample chamber fill capillary, which consisted of a $40 \mathrm{~cm}$ length of $0.12 \mathrm{~mm}$ 1.d. capillary tubing from the vacuum can 
flange to the $\mathrm{He}^{4}$ pot, was soldered into $40 \mathrm{~cm}$ of $0.8 \mathrm{~mm}$ i.d. copper refrigerator tubing which was wound in a helix from the top to the bottom of the pot. A similar helically-wound section of copper tubing was wound with this $\mathrm{He}^{4}$ capillary and used as part of the $\mathrm{He}^{3}$ pot return capiliary. The He ${ }^{4}$ pot was filled directly from the $4.2 \mathrm{~K}$ bath through an $11 \mathrm{~cm}$ length of $0.3 \mathrm{~mm}$ i.d. cupronickel tube which passed from the $4.2 \mathrm{~K}$ helium bath through the vacuum can flange and hence down into the pot. The upper end of this fill capillary was closed with a valve which consisted of the stainless steel stem of a commercial needle valve (Hoke type $1325 \mathrm{M} 4 \mathrm{~B}$ ) and a brass seat with a $0.079 \mathrm{~cm}$ hole through it into which the valve stem was seated.

Measurements of the volume of gas pumped from the He ${ }^{4}$ pot made with a gas flow meter showed that under typical running conditions the loss rate was $10^{-3}$ ifters NTP/sec, which corresponds to a heat leak into this pot of $4.3 \mathrm{~mW}$. Using either the remote pump or the pump in the room, it was found that the lowest $\mathrm{He}^{4}$ pot temperature was around $1.25 \mathrm{~K}$. 5. The $\mathrm{He}^{3}$ Pot

The $\mathrm{He}^{3}$ pot had a volume of approximately $10 \mathrm{~cm}^{3}$. This pot was pumped through a $4.07 \mathrm{~cm}$ length of 
$0.795 \mathrm{~cm}$ i.d. stainless steel tubing. This tube led to a length of $3.49 \mathrm{~cm}$ diameter stafnless steel tubing which connects at room temperature to the demountable valve mentioned earlier, and then to the external $\mathrm{He}^{3}$ pumping system.

The $\mathrm{He}^{3}$ pot could be pumped in either one of two ways. While at rest, the $\mathrm{He}^{3}$ pot could be connected to the external $\mathrm{He}^{3}$ pumping system which consisted of a two inch diffusion pump backed by a sealed mechanical pump. The sealed pump exhausted into a $\mathrm{He}^{3}$ gas storage vessel.

Since we also wished to be able to run at $\mathrm{He}^{3}$ temperatures while in rotation, and we wished to avoid using rotating pumping seals to pump the valuable $\mathrm{He}^{3}$ gas, a second method of pumping the $\mathrm{He}^{3}$ was used when the system was in rotation. In this second pumping method, a cylindrical cage of kidney-shaped cross section, $10 \mathrm{~cm}$ long, filled with molecular sieve (Linde type $13 \mathrm{X}$ ), was used. The cage was kidney shaped so that it could be raised and lowered in the $3.49 \mathrm{~cm}$ pumping $11 \mathrm{ne}$ and would clear the tensioncontrol rods which occupied the center of this tube. This type of pump is called an adsorption pump. When the molecular sieve in the pump was cooled to $4.2 \mathrm{~K}$, $\mathrm{He}^{3}$ vapor would be adsorbed on 1 ts surface at a rate 
which corresponds to an equivalent pumping speed faster than that of the external diffusion pump when it was used pumping through its connecting lines. The $30 \mathrm{grams}$ of molecular sleve used was sufficient to adsorb all of the 6 NTP 1 iters of $\mathrm{He}^{3}$ gas when the sieve was cooled to $4.2 \mathrm{~K}$.

The molecular sleve cage was connected to a winch at room temperature using 2 pound-test nylon monofilament fishing tackle. This winch was used to raise and lower the sieve. When the sleve was raised to room temperature, the adsorbed $\mathrm{He}^{3}$ gas outgassed from the sleve rapidiy, leaving the gas and sieve available for reuse.

In using this sieve, one precaution was necessary. The sleve had to be heated to an elevated temperature and pumped upon prior to use in order to clear it of adsorbed gases. The gases, if not baked out, reduced the amount of $\mathrm{He}^{3}$ vapor which the sieve could adsorb to a point where the sieve was not useful. The $\mathrm{He}^{3}$ pot could be ftlled in two ways. The vapor could be condensed from room temperature down the $\mathrm{He}^{3}$ pot pumping $11 \mathrm{ne}$, or it could be condensed into the $\mathrm{He}^{3}$ pot through a small capillary which is not shown in Fig. 3. An advantage to condensing through the small capillary is that the $\mathrm{He}^{3}$ pot 
itself could be pumped while the condensation took place. Then, if the $\mathrm{He}^{3} \mathrm{gas}$, which passed through the sealed pump, was exhausted into the small f 111 capillary and recondensed, a closed cycle was set up, and the $\mathrm{He}^{3}$ refrigerator was operating in the socalled recirculating mode. This return capillary was chosen so that its impedance to the flow of 1iquid $\mathrm{He}^{3}$ at temperatures less than $1 \mathrm{~K}$ was just large enough to allow slightly more $\mathrm{He}^{3}$ liquid to enter the $\mathrm{He}^{3}$ pot than was pumped out. At our largest heat leak or when the pot was at a temperature near the $\mathrm{He}^{4}$ pot temperature, the $\mathrm{ratio}$ of $\mathrm{He}^{3}$ returned to that pumped away was approximetely 1. In our case, for our heat leak, the proper rate of $\mathrm{He}^{3}$ return was achieved by passing a $0.1 \mathrm{~mm}$ wire through the entire $10 \mathrm{~cm}$ length of $0.128 \mathrm{~mm} 1 . \mathrm{d}$. capillary soldered to the outside of the $H e^{3}$ pot. Under actual running conditions, this system would allow anywhere from $40 \%$ to $60 \%$ of the $\mathrm{He}^{3}$ to be in the $\mathrm{He}^{3}$ pot as 11quid, the exact amount depending on bath level.

The walls of the $\mathrm{He}^{3}$ pot were used to thermally ground all electrical leads and fill capillaries going to the sample chamber.

In addition, passing through the He ${ }^{3}$ pot was 
the tension-control rod. This rod was screwed into the diaphragm in the center of the stafnless steel piece which sealed, by means of an indium seal, the bottom of the $\mathrm{He}^{3}$ pot. This tension-control rod was cooled at its lower end by the He $\mathrm{H}^{3}$ liquid in which it was bathed.

Measurements were made of the rate at which He ${ }^{3}$ gas was collected in the $\mathrm{He}^{3}$ gas storage can at room temperature under various running conditions. Under typical running conditions, with $\mathrm{He}^{4}$ in the sample chamber and the tension-control rod in place, the average heat leak, as deduced from the gas recovery rate, was $122 \mu \mathrm{W}$. In another run, without the sample chamber or tension-control rod in place, the heat leak deduced by the same method was $60 \mathrm{HW}$. The tension-control rod was the single largest source of heat leak into the pot.

The lowest temperature achleved under typical running conditions depended upon the pumping method used. When the external diffusion pump was used, the lowest temperature was approximately $0.5 \mathrm{~K}$. When the molecular sleve pump was used under the same conditions, the lowest temperature was approximately $0.4 \mathrm{~K}$. 


\section{Thermometry}

Thermometry was carried out using the vapor pressure of $\mathrm{He}^{4}$ gas as a standard for temperatures above $1.2 \mathrm{~K} .(47)$ For lower temperatures the vapor pressure of the $\mathrm{He}^{3}$ gas was used. (48) Speer carbon resistors were attached to the sides of both the $\mathrm{He}^{4}$ pot and the $\mathrm{He}^{3}$ pot, and also to the side of the sample chamber, and were used as secondary thermometers. Calibrations of these thermometers at temperatures above $1.2 \mathrm{~K}$ was done during our set-up runs. In these runs the resistors were in direct contact with the pumped bath. Further, the vapor pressure of the bath was easily obtained using an Octoil manometer. Calibration at lower temperatures was done during a regular run, using a McLeod gauge to measure the vapor pressure of the $\mathrm{He}^{3} \mathrm{gas}$. In a typical run, only the He ${ }^{4}$ pot was pumped, the $\mathrm{He}^{3}$ vapor standing above the liquid in the $\mathrm{He}^{3}$ pot being used only as an exchange gas to assure thermal equilibrium between the $\mathrm{He}^{3}$ pot and the $\mathrm{He}^{4}$ pot. The sample chamber was connected to the $\mathrm{He}^{3}$ pot by a copper wire. The observed temperature drift during a run was at most $15 \mathrm{mK}$, over a time period of some 17 hours. Since the only explicit temperature dependence in the experiment is in the 
superfluid density, and at $1.3 \mathrm{~K}$ a $15 \mathrm{mK}$ drift has a negligible effect on the value of the superfluid density, it was not necessary to regulate the temperature.

The resistance of a resistance thermometer was measured with an a.c. Wheatstone bridge with the thermometer resistor as one arm of the bridge.

\section{Measurement of Rotation Speed}

Measurements of the rotation speed of the turntable were made using a stationary photoconductive cell mounted on the dewar stand and a stationary light mounted on the dewar stand. The beam from the light was reflected by a mirror mourted on the turntable and rotating with it. Each time the reflected light beam flashed upon the photo-cell, the cell became conducting, resulting in a voltage drop which was amplified and used to drive a bistable multivibrator. On the first pass, the output of the multivibrator was used to start a time-interval counter, and on the second pass it was used to stop the timeinterval counter. In steady rotation the time interval measured was stable to less than $0.5 \%$.

While this method was useful for measuring the average rotational speed over a single revolution, 
it was not particularly sensitive to possible jerks in the turntable's motion as the turntable rotated. Bvery effort was made to eliminate these ferks, since their presence gave rise to unwanted excltations of the wire.

\section{Tension Control}

When the wire was encapsulated in the sample chamber, inside the brass can, the only wire property over which control could be exercised was the wire tension. The wire twist was already preset, using the method described in Appendix II.

It was extremely important to have a stable wire tension, since small changes in the wire tension, by changing the wire frequency, could bring about changes in the intrinsic frequency splitting. Of course, it was necessary to maintain a constant intrinsic frequency splitting in order to extract the apparent circulation from the measured total frequency splitting. Another important reason to maintain a constant running frequancy concerned the automatic data-acquisition system. The phase-1ock loop used in that system could track frequency shifts only if they were limited to approximately $\pm 0.5 \%$. Thus, the tension had to be maintained to $\pm 1 \%$. 
Two different tension control systems were used in the course of the measurements. In both systems a screw assembly, which was located on the axis of the $\mathrm{He}^{3}$ pumping 1 ine and in the plane of the vacuum can flange, was used. The assemblies were kept at the bath temperature, $4.2 \mathrm{~K}$, to prevent undesirable changes in tension due to thermal expansion end contraction.

The first was a direct screw control, using a $1 / 4 \mathrm{in}$. $x 60$ screw thread. This control was coarse, but fine adfustments could be made using a lever arm fixed to the tension-control rod and driving this lever arm with a screw. With this system it was an extremely tedious process to set the proper tension, as determined by measuring the wire frequency. However, once the wire frequency desired was obtained, the wire would remain at that frequency to within $\pm 0.2 \%$. Only sharp jolts delivered directly to the tension-control rod or liquid nitrogen transfers were found to change the frequency. The tedious nature of the frequency setting process and the changes brought about by nitrogen transfers led us to try another tension-control system. This system utilized a differential screw. In this screw the outer thread was a $5 / 16$ in. $x 40 \mathrm{screw}$, 
while the inner thread was $11 / 64$ in. $x 38$, producing an equivalent screw of 760 threads per inch. In addition to changing the type of screw, the means used to couple the screw to room temperature was changed. Instead of an unbroken rod leading from the screw to room temperature, a tine at the end of the rod from room temperature engaged a slot at the end of the center, movable piece of the differential screw. In this way, the screw could be turned, but contraction and expansion of the connecting rod from room temperature would not affect the tension. This system gave a more sellsitive control over the tension, but the frequency was sot nearly as stable over long times as it was with the first system used. This instability made necessary frequent readjustments of the wire frequency and proved to be very tedious.

Nefther method was satisfactory.

9. Mercury S1ip Rings

Since most of the measurements were made while the apparatus was in rotation, it was necessary to electrically couple the rotating apparatus with the stationary electronics. This was accomplished by using mercury slip rings. Eight slip rings were available in the unft used. Two were used for the 
wire signal, three for thermometry, and two were used for the direction-finder magnet.

\section{Electrical Apparatus}

An overall block diagram of the electrical apparatus used in this experiment is shown in Fig. 11. Included in the figure is the automatic data-taking system used during the later runs of the experiment.

The sequence of events for a single circulation measurement begins with the negative-going sawtooth signal at the waveform generator. This negativegoing sawtooth was used to trigger pulses from three pulse generators. Pulse generator number one produced a positive pulse of approximately $8 \mathrm{msec}$ in length. This pulse would open gate number one and allow the square-wave signal generated in the oscillator (VCO) to pass through the bridge to drive the wire. The bridge is a Wheatstone bridge with the wire as one arm. The bridge was transformercoupled at both its input and output to prevent bothersome ground loops. This same pulse from pulser number one was also used to trigger the two ascilloscopes used to monitor the wire signal and also to start the time interval counter used in the automatic data-taking system. After some $8 \mathrm{msec}$ 
the pulse would end, the gate would close, and the wire would oscillate freely.

The emf generated by the freely oscillating wire would then be fed from the bridge into two independent monitoring circuits. In the first of these circuits the signal would be amplifled by a wide-band amplifier and displayed on the screen of the oscilloscope. This display was used to monitor the wire output through the bridge during the drive period in order to check the bridge balance. This amplified signal was also fed to gate number two, which would be opened by pulse number two approximately $100 \mathrm{msec}$ after thc wire drive began. This pulse was sufficiently long to allow the counter to take a ten-period average of the wire period, thus giving a pulse-by-pulse measurement of the wire period. Using this measurement, the wire could be kept in tune at the desired running frequency.

The signal from the bridge was also fed into a low-noise pre-amp of gain 100 (PAR type 112) and from there into the signal channel of a lock-in amplifier (PAR model 120). The signal channel of the lock-in amplifier consists of a variable-gain wide-band amplifier in series with a tuned amplifier. 


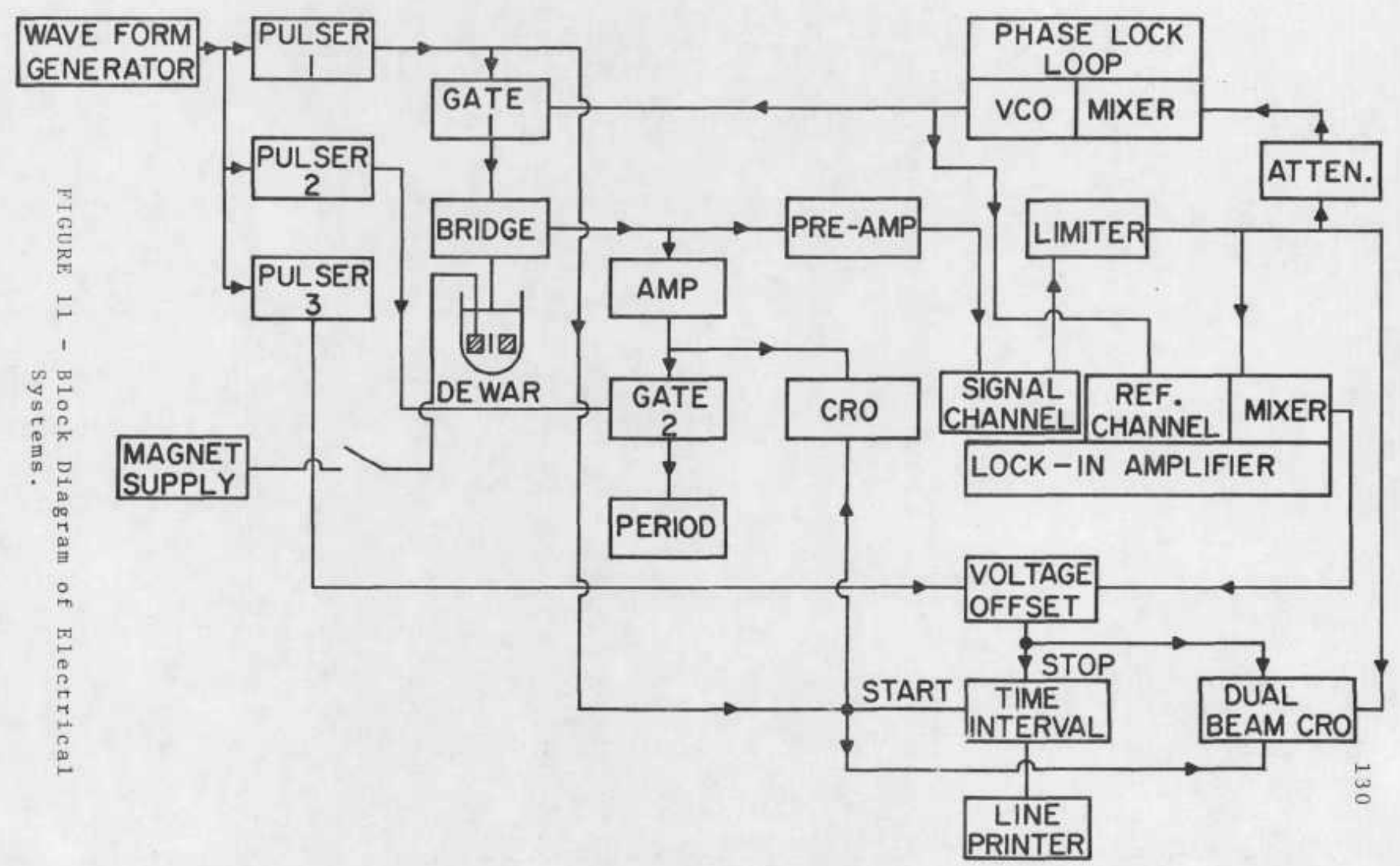


The $Q$ of the tuned amplifier is 10 , A tuned amplifier is used because of its high noise refection.

The signal from the tuned amplifier was displayed on the screen of an oscilloscope, and if the sweep speed of the scope were calibrated, this signal could be used for direct visual measurement of the time to the node in the wire beat pattern. As an alternative, the signal could be fed into the automatic data-recording system and be used to generate the signal which would turn off the time-interval counter.

\section{Automatic Data System}

Because we wished to take data every six seconds while the system was being continuously accelerated, and the acceleration took place over many hours, an automatic data-acquisition system was designed. This system consisted of a lock-in amplifier, a phase-locked loop, a time-interval counter, and a line printer.

The basic component of the automatic data system was the lock-in amplifier. The emf generated by the oscillating wire was fed into the signal channel of the lock-in amplifier. This emf may be written, from Eq. 2-68, in the form $E=C e^{-\lambda t} \cos \left(\frac{\Delta \omega}{2} t\right) \sin (\omega t)$. If this signal is fed into the mixer of a lock-in 
amplifier whose reference frequency is $\omega$, the output of the mixer is a d.c. voltage given by

$$
v_{0} a e^{-\lambda t} \cos \left(\frac{\Delta \omega}{2} t\right)
$$

We note that after a time $t_{n}=\pi / \Delta \omega_{t}$ this signal passes through 0 volts and changes sign. Also, this time $t_{n}$ is the time to the first node in the beat pattern and is the quantity we wish to measure. It was this passage of the voltage output of the lock-in amplifier through zero which was utflized in the automatic system. An electronic counter (Hewlett-Packard type $5233 l$ ) in the time-interval mode was turned on by the output of pulser number one. This counter would then count until the passage of the voltage output of the lock-in through zero. This event would then terminate the interval of time being counted. This time interval, the interval between the initiation of the wire excitation and the occurrence of the first node in the beat pattern, would then be printed automatically by a digital recorder (Hewlett-Packard type 562A).

In order for this system to function properly the wire frequency had to be stable. When the wire signal was monftored by a counter throughout the oscillation, however, it was found that the wire's 
frequency was not stable. During the approximately one-second time that it took for the wire signal to decay to nolse, the frequency drifted by a maximum of $\delta \omega \approx \pm 6 \mathrm{rad} / \mathrm{sec}$. Because of this drift the output signal of the lock-in is given by

$$
\nabla_{0} \alpha e^{-\lambda t} \cos \left(\frac{\Delta \omega}{2} t\right) \cos (\delta \omega t)
$$

We note that with $\delta_{\omega}=6 \mathrm{rad} / \mathrm{sec}$ the second cosine factor goes through zero in a time given by $t_{0}=$ $\frac{\pi}{2 \delta \omega}$ or $t_{0} \approx 300 \mathrm{msec}$. In general, if $\delta \omega$ is greater than $\frac{\Delta \omega_{t}}{2}$, a spurious zero crossing occurs, and the system does not function as desired.

Since there was no way to further stabilize the wire frequency, a way was found to produce a reference signal which tracks the wire in frequency. To do this we used a Signetics 561B phase-1ock loop (PLL).

The wire signal, as derived from the output of the signal channel of the lock-in amplifier, was limited and attenuated, and then fed into the mixer of the PLL. The output of the voltage-controlled oscillator (VCO), which is part of the PLL, was fed into the PLL mixer as well. If these two signals differ in phase, an error signal is generated in the PLL mixer which, after filtering, is fed back 
to the VCO to bring its phase into confunction with the signal being monitored. Thus the PLL, taken as a unit, acts as an oscillator whose frequency tracks the frequency of another signal, in our case, the wire signal.

The filtering of the output signal from the PLL mixer determines the rate at which the Vro frequency responds to the changes in the input signal frequency. Under most running conditions a filtering time constant of $40 \mathrm{msec}$ was found to be quite adequate.

The output signal from the VCo was used as the reference signal in the lock-in ampiffier. The output of the lock-in, with this reference signal, was a d.c. signal which passed through zero at the time of the first node in the beat pattern. This zero crossing was used, as was mentioned before, to turn off the time-interval counter.

This system worked very we11. Occasional1y, while the wire was being driven, a spurious zero crossing occurred, usually if the bridge had slipped slightly out of balinee. To prevent these eretestabth from disturbing our measurements, a voltage offset was imposed on the output of the mixer during the time the wire was being driven. This voltage 
offset was provided by pulser number three.

The amplitude of the wire signal varied by a factor of 100 from excitation to the null in the beat signal. In order to prevent overloading of the output amplifier of the lock-in during the early part of the wire signal, a limiter was interposed between the output of the signal channel of the $10 \mathrm{ck}-i \mathrm{n}$ and the input to the mixer of the lock-in. This limiter limited the amplitude range of the signal, allowing a maximum signal amplitude only ten times that of the minimum signal amplitude.

\section{Tuned Amplifier}

As mentioned above, a tuned ampliffer was used as part of the measurement system. While it was essential to use this amplifier to reject noise, its introduction into the system did complicate the measurement.

We may model a tuned amplifier as a damped harmonic osciliator befing driven by the incident signal, the signal from the wire. Since the incident signal is a superposition of two sinusoidal signals of slightly different frequency, the amplifier as a resonant system shifts the phases of these signals by different amounts. Since it is the steadily changing phase difference between these 
signals which produces the beat pattern, this unequal shift in the phases of the incident signals produces a change in the time of the appearance of the first node in the beat pattern, this change being found to be a delay.

If the input signals are of frequency $\omega^{+}$and $\omega^{-}$respectively, proper tuning of the amplifter occurs when it is tuned to a frequency given by $\omega_{0}^{2}=$ $\omega^{+} \omega^{-}+2 \lambda(\gamma-\lambda)$ where $1 / \gamma$ is the time constant for free decay of the amplifier and $1 / \lambda$ is the time constant for wire decay. It is assumed $1 / \gamma \ll 1 / \lambda$, a condition easily satisfied experimentally. When the amplifier is properly tuned the node is a true nu11. At resonance the node appears at a time which is later than the time to the node in the absence of the amplifier, the amount of the time delay being given approximately by

$$
\tau=1 /(\gamma-\lambda)\left[1-1 / 12\left(\frac{\Delta \omega}{\gamma-\lambda}\right)^{2}\right],
$$

where $\Delta \omega=\omega^{+}-\omega^{-}$. Under typical running conditions $\frac{\Delta \omega}{\gamma-\lambda}$ was 0.05 in maximum value. Thus, $(\gamma-\lambda)^{-1}$ was, in all cases, the time delay to $1 \%$ accuracy.

Experimentally, the amplifier had to be properly tuned and the time delays of the amplifier and wire had to be determined. The amplifier was tuned by observing the Lissajous figure produced on the screen 
of the oscilloscope. A steady signal at the same frequency as the wire signal was introduced into the amplifier. The output signal was imposed on the horizontal plates of the oscilloscope and the input signal was imposed on the vertical plates. The amplifier was tuned so that the correct phase shift of $0^{\circ}$ was observed. After this tuning the decay time of the amplifier was measured by introducing a $5 \mathrm{~V}$, 1 usec pulse into the amplifier and measuring the time for the resultant ringing signal at the output to decay by a factor of $\frac{1}{2}$ in amplitude. The decay time $1 / \lambda$ is then the halving time divided by 0.69 . In a similar way, the wire was excited by a $5 \mathrm{~V}$, 1 msec pulse and its halving time measured. Of course, the wire decay time was measured using a wide-band amplifier.

\section{Magnets}

Two magnets were used in the experiment. One was used to provide the main field for exciting and sensing the wire's motion while the other was used to provide the perturbing field for measuring the sense of the circulation.

The main magnet was a superconducting magnet operated in a persistent mode.

The magnet was designed to provide a field 
strength of $2000 \mathrm{G}$ at the wire when the winding was carrying a 2 A current. The winding consisted of 2000 turns of superconducting wire (Supercon type $A-25)$.

The persistent current switch consisted of 10 turns of superconducting wire surrounded by approximately 50 turns of Evanohm wire, having a resistance of approximately $1500 \Omega$. The superconducting wire and the Evanohm wire were wound on a Bakelite spool.

The perturbing-field magnet consisted of 200 turns of the same superconducting wire. It was designed to provide a field of $200 \mathrm{G}$ in a direction perpendicular to the main field when it was carrying a 1 A current.

The perturbing field was switched on manually while the wire was being exclted and then switched off, so that the free oscillation took place in the presence of the main field only. 


\section{APPENDIX II}

\section{WIRE PREPARATION}

\section{Making The Wire}

The wires used in the experiment were goldplated quartz fibers. They were formed by heating a $3 / 16$ in. quartz rod in an oxygen-gas flame. The ends of the rod were then rapidly drawn apart, leaving a thin quartz fiber connecting them.

The fibers were measured for thickness, using a micrometer. Only fibers whose thickness was less than 125 um were selected.

Using a microscope with a calibrated reticle, mutually perpendicular diameters of the fibers were measured. Of the fibers measured, only those whose diameters were matched to about $5 \%$ were chosen for gold-plating. Typically, from a batch of 200 fibers of six-inch length fewer than 20 would meet these st andards.

The fibers chosen were then mounted on a spit and placed in a vacuum system. The pressure in the system was reduced to less than $10^{-6}$ Torr, and while the spit was rotating gold was evaporated onto the fiber. Care had to be taken not to evaporate too much gold onto the fiber, as it was found that a 


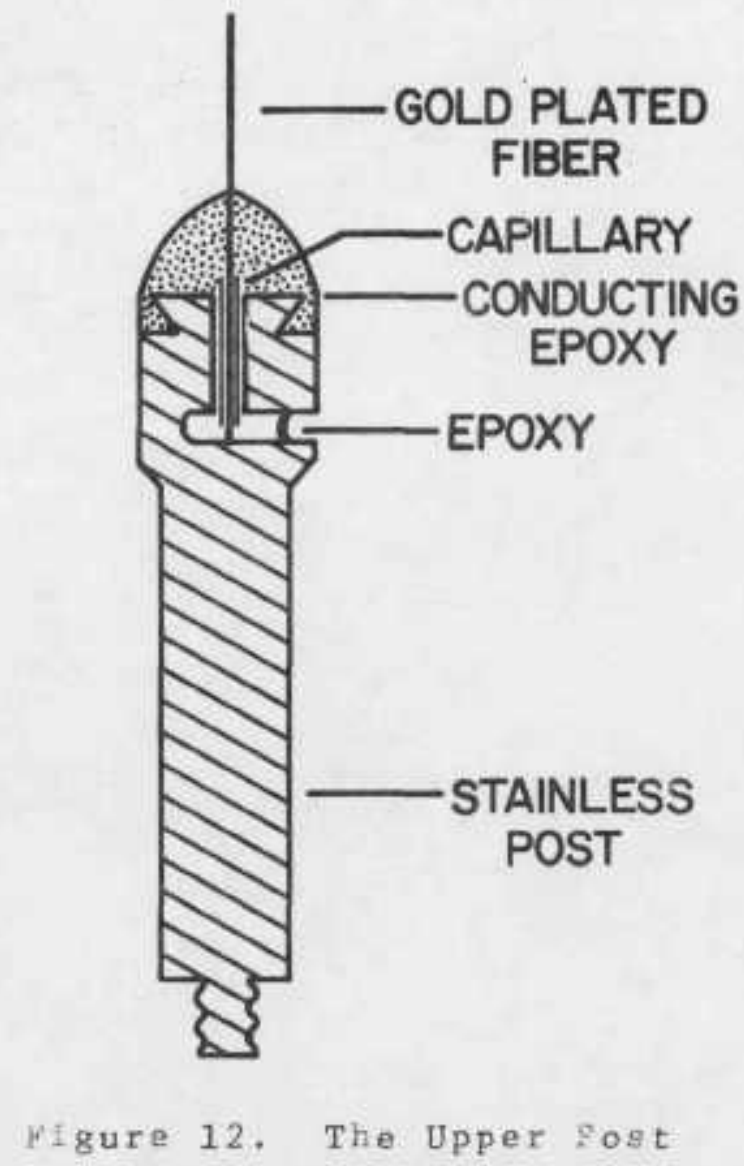

thick gold plating would not adhere to the fiber when it was mounted for an experimental run and cooled. The gold thickness, as estimated from the amount of gold used in the evaporation, was around $700 \stackrel{\circ}{\mathrm{A}}$.

2. Mounting The Wire

After the fiber was plated, it was cut into

$61 \mathrm{~mm}$ lengths. The gold was scraped from the ends of the fiber and the ends of the fiber were inserted into short pieces of $0.3 \mathrm{~mm}$ i.d. capillary tubing which had been placed in the ends of the posts to 
which the fiber was to be epoxied as shown in Fig. 12. A household epoxy (Epoxy 220) was then placed Into the transverse hole in the post and allowed to cure. Since the gold was scraped from the ends of the fiber, this epoxy bond was made directly to the quartz. After this epoxy cured, a second epoxy (Epoxy Products type 3021), which was conducting, was applied to the ends of the posts and shaped to form as smooth a surface as possible. This epoxy formed a conducting link between the fiber and the post, and made possible the electrical drive and electrical sensing of the wire's motion.

After the conducting epoxy cured, the threaded end of the upper post was screwed into a mounting post which was silver soldered into the center of the $.004 \mathrm{in.}$. stainless diaphragm indicated in Fig. 2 . The wire and its end posts were then placed into a quartz-glass-and-brass support tube which supported it during the experiment. The bottom post was clamped to the twist assembly shown in Fig. 2 by set screws. The twist assembly was clamped to the bottom end of the support tube, also by set screws. This supporting tube, the wire, and the stainless 
steel end piece containing the feed-through ports then formed a self-contained unit.

At this point in the assembly procedure, this unit would be cooled to liquid nitrogen temperatures a number of times and checked to make sure that electrical continuity was maintained.

After electrical continufty at low temperatures was assured, the wire and its support tube assembly would be mounted on the apparatus. The end cap of the He ${ }^{3}$ pot had a stainless steel diaphragn. In the center of this diaphragm was a short length of threaded rod. This rod was screwed into the post in the center of the diaphragm to which the wire was attached. This double diaphragm systen was connected directly to the wire tension-control rod (see Fig. 3) and was flexible enough to allow tension changes in the wire which extended from zero (slack wire) to the wire's breaking point, a total motion of $0.05 \mathrm{~mm}$.

\section{Wire Set-Up Run}

Under full running conditions the wire and support tube were enclosed in a brass can. Under these conditions the only adjustment that could be made on the wire was a change in tension. However, both Vinen and Whitmore discovered in 
their work that the intrinsic frequency splitting of the wire could be reduced if the wire were twisted, one end relative to the other. To set the twist in the wires used in this work, a separate run, called a set-up run, was made. This run is described below.

After the wire and support tube were mounted on the bottom of the $\mathrm{He}^{3}$ pot, the twist assembly in the bottom of the support tube was coupled to a gear. The set screws coupling the twist assembly to the bottom of the support post were loosened, thereby allowing the bottom post to be freely rotated relative to the top post.

The twist-assembly gear system was connected to the end of a can which bolted to the vacuum can flange, but whose length was such that the end of the wire support tube would just stick out its bottom. A pinon gear which could be turned from the room temperature flange engaged the wire twist gear, thus allowing the wire to be twisted when the apparatus was placed in the dewar.

A permanent magnet was placed around the end of the can to allow for excitation and observation of the wire's oscillation. 


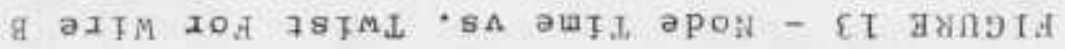

TIME TO NODE IN MILLISECONDS

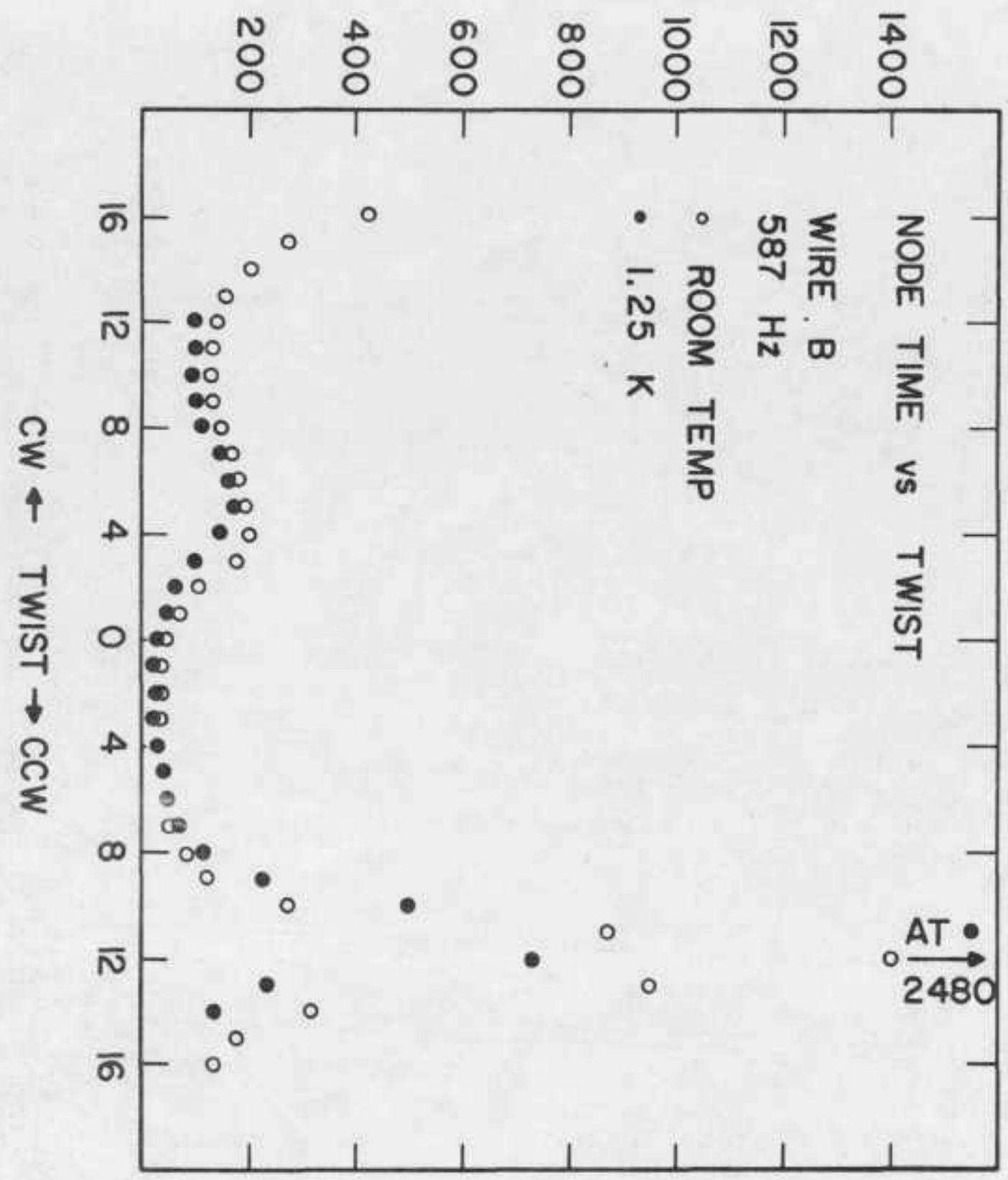


It was found that by testing the room temperature behavior of the intrinsic frequency splitting as a function of wire twist, the low temperature behavior could be predicted. The intrinsic frequency splitting at a particular frequency was measured in vacuum at room temperature and if it were promising, the low temperature properties would be studied. This was done by transferring 1iquid helium and cooling the system to $1.2 \mathrm{~K}$.

A comparison of the room temperature behavior and the low temperature behavior of the intrinsic frequency splitting as a function of twist is shown for wire B in Fig. 13.

At low temperatures, during this set-up run, the wire twist was set such that the node could be seen clearly just before the wire oscillation damped into the noise, a time of about $1200 \mathrm{msec}$. The system was then allowed to warm to room temperature, and the set screws which clamped the twist assembly were tightened.

A comparison of the frequency splitting as a function of frequency for this wire before and after the set screws were tightened is shown in Fig. 14. Of course, these data were taken in two 


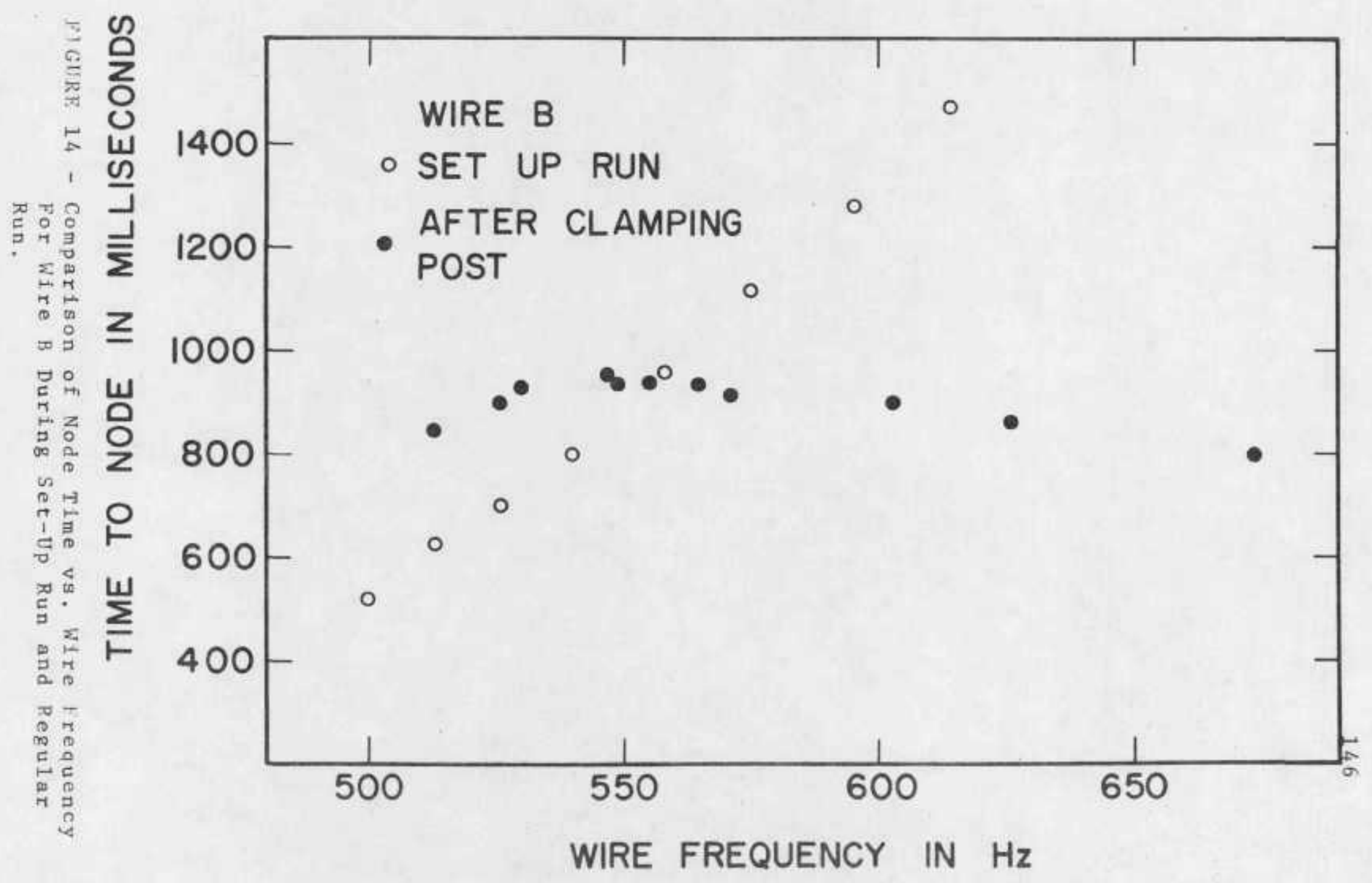


completely different runs, and it is not known, in either case, if circulation was present around the wire. However, both sets of data were taken at low temperature, and more importantly, the changes exhibited are typical of what was seen in every case.

It is not surprising that some change is seen. The process of clamping the lower post with set screws could certainly alter the position of the lower post with respect to the upper post. However, we would expect that a small shift in position of the lower post would simply change the slope of the curve, and that there would still be a frequency at which the node disappeared into the noise. The fact that this is not what was seen suggests that something else is happening. The last run with wire $C$, in which persistent rapping of the apparatus produced a node-time versus frequency curve with a very steep slope suggests that in some way one of the posts was sticking, and that this rapping was bringing about conditions more nearly like those in our set-up run.

It would be interesting to try runs in which no motion of the post was allowed. A rigid system, 
If properly tuned in a set-up run, could help avoid these problems.

Finally, it should be noted that during these set-up runs it was possible to measure circulation. These measurements were made under circumstances which were very similar to those of Whitmore. In our case, a set-up run would last twelve hours, after which the helium level would fall below the top of the wire. The circulation values measured during these twelve hours were very unstable, changing frequently. These results are consistent with Whitmore's results. 


\section{REFERENCES}

1. L. D. Landau, Phys. Rev. 60,357 (1941);

J. Phys. (U.S.S.R.) 5, 71 (1941); J. Phys.

(U.S.S.R.) $8,(1944) ;$ J. Phys. (U.S.S.R.)

11, 91 (1947); Phys. Rev. 75,884 (1949).

2. D. V. Osborne, Proc. Phys. Soc. (London) $\mathrm{A63}$, $909(1950)$.

3. E. L. Andronikashvili and I. P. Kaverkin, Soviet Physics J.E.T.P. 1, 174 (1955).

4. R. P. Feynman in Progress in Low Temperature Physics, Vo1. I, edited by C. T. Gorter (North Holland, Amsterdam, 1955).

5. L. Onsager, Nuovo Cimento 6 , Supp. $\underline{2}, 249$ (1949).

6. G. W. Rayfield and F. Reif, Phys. Rev. 136, A1194 (1964).

7. G. Gamota and T. M. Sanders, Phys. Rev. Letters 15, 949 (1965).

8. P. L. Richards and P. W. Anderson, Phys. Rev. Letters 14, 540 (1965).

9. H. E. Hall and W. P. Vinen, Proc. Roy. Soc. (London) A238, 204 (1956).

10. W. F. Vinen, Proc. Roy. Soc. (London) A260, 218 (1961).

11. S. C. Whitmore and W. Zimmermann, Jr., Phys. Rev. 166,181 (1968).

12. Reference 10.

13. S. C. Whitmore, Ph.D. Thesis, University of Minnesota, 1966 (unpub1ished).

14. G. G. Stokes, Mathematical and Physicsl Papers (Cambridge, 1901) Vol. 3, p. 38 . 
15. J. T. Stuart, in Laminar Boundary Layers, edited by L. Rosenhead (Clarendon Press, Oxford, 1963) p. 390.

16. M. Abramowitz and $I$. Stegun, Handbook of Mathematical Functions (N.B.S. Appl. Math. Series 55, Washington, D.C., 1964) p. 358.

17. E. Jahnke and F. Emde, Tables of Functions (Dover, New York, 1945) 4th ed., p. 252.

18. L. A. Sege1, Quart. App1. Math. 18, 335 (1961).

19. R. P. Kanwa1, Z, angew. Math. Mech. 35 , 17 (1955).

20. H. Lamb, Hydrodynamics, 6th ed., (Cambridge University Press, 1932 , reprinted by Dover, New York, 1945) p. 83.

21. Reference 20 , p. 78 .

22. Reference 20 , p. 681 .

23. Reference 13, p. 29.

24. P. M. Morse, V1bration and Sound 2nd ed., (McGraw-H111, New York, 1948) p. 166.

25. Reference 24.

26. K. R. Symon, Mechanics 2nd ed. (AddisonWesley Publishing Company, Inc., Reading, Mass., 1960) p. 271.

27. A. L. Fetter and K. Harvey, Phys. Rev. 14 , 2305 (1971).

28. R. Courant and D. Hilbert, Methods of Mathematical Physics (Interscience, New York, 1953) Vo1. 1, p. 363.

29. Reference 13. 
30. J. M. Reynolds, R. G. Hussey, D. P. Thibodeux,

B. F. Tucker, and R. F. Folse, "The oscillations of Cylinders and Spheres in Liquid

He II", Tech. Doc. Report ML TDR 64-314

(Louisiana State Univ., 1964); see also

R. G. Hussey, B. J. Good, and J. M. Reynolds, Phys. Fluids, $10,89(1967)$.

31. Reference 10.

32. Reference 30 .

33. J. G. Dash and R. D. Taylor, Phys. Rev. 105 , 7 (1957).

34. J. Topping, Errors of observation and Their Treatment (Reinhold Publishing Co., New York, 1957) p. 82 .

35. L. D. Landau and E. M. Lifshitz, Statistica1 Physics (Addison-Wesley, Reading, Mass., 1958) p. 72 .

36. A. L. Fetter, Phys. Rev. 153, 285 (1967).

37. Reference 36.

38. A. L. Fetter, Phys. Rev. 152, 183 (1966).

39. D. Stauffer and A. L. Fetter, Phys. Rev. 168, 156 (1968).

40. V. K. Tkachenko, Soviet Physics (J.E.T.P.) 22, $1282(1966)$.

41. Reference 39.

42. Reference 39.

43. J. A. Northby and R. J. Donnelly, Phys. Rev. Letters $25,214(1970)$.

44. D. S. Shenk and J. B. Meh1, Phys. Rev. Letters 27,1703 (1971).

45. D. J. Griffiths, Proc. Roy. Soc. (London) A277, $214(1964)$. 
46. Reference 11.

47. National Bureau of Standards, Monograph 10 $(1960)$.

48. R. H. Sherman, S. G. Sydoriak, and

T. R. Roberts, J. Res. Nat1. Bur. Std. (U.S.) 68A (Physics \& Chemistry) 579 (1964). 


\section{ACKNOWLEDGMENTS}

I wish to express my graditude to my advisor, Professor William Zimmermann, Jr., for his counsel and aid throughout this experiment.

I also thank those other members of the faculty of the University of Minnesota who aided me in this work. In particular, I wish to thank Professor Walter Weyhman for his many valuable suggestions which helped in the construction of the apparatus. In addition, I wish to thank Professor Michael Moldover for his advice and friendiy encouragement.

I thank my fellow graduate students who helped during the experiment and made the many hours in the laboratory a pleasant time. In particular, I wish to thank Mr. Brian Sabo for his many contributions, and Mr. James McLinn who aided in some of the measurements.

Finally I wish to thank my wife Ann, without whose love, understanding, and patience this work would never have been done. 